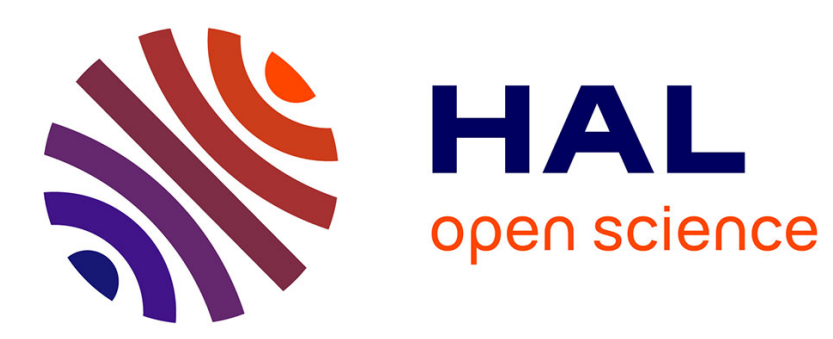

\title{
A newcomer's guide to ultrashort pulse shaping and characterization
}

\author{
Antoine Monmayrant, Sébastien J. Weber, Béatrice Chatel
}

\section{To cite this version:}

Antoine Monmayrant, Sébastien J. Weber, Béatrice Chatel. A newcomer's guide to ultrashort pulse shaping and characterization. Journal of Physics B: Atomic, Molecular and Optical Physics, 2010, 43 (10), pp.103001. 10.1088/0953-4075/43/10/103001 . hal-00569786

\section{HAL Id: hal-00569786 \\ https://hal.science/hal-00569786}

Submitted on 25 Feb 2011

HAL is a multi-disciplinary open access archive for the deposit and dissemination of scientific research documents, whether they are published or not. The documents may come from teaching and research institutions in France or abroad, or from public or private research centers.
L'archive ouverte pluridisciplinaire HAL, est destinée au dépôt et à la diffusion de documents scientifiques de niveau recherche, publiés ou non, émanant des établissements d'enseignement et de recherche français ou étrangers, des laboratoires publics ou privés. 


\title{
A newcomer's guide to ultrashort pulse shaping and characterization
}

\author{
Antoine Monmayrant ${ }^{1,2}$, Sébastien Weber $^{3}$, and Béatrice \\ Chatel $^{3}$ \\ ${ }^{1}$ CNRS-LAAS ; 7 avenue du colonel Roche, F-31077 Toulouse, France \\ ${ }^{2}$ Université de Toulouse ; UPS, INSA, INP, ISAE ; LAAS, F-31077 Toulouse, France \\ ${ }^{3}$ CNRS-Université de Toulouse; UPS, Laboratoire Collisions, Agrégats Réactivité, \\ IRSAMC, F-31062 Toulouse, France. \\ E-mail: beatrice.chatel@irsamc.ups-tlse.fr
}

\begin{abstract}
This tutorial gives an overview of the most widespread techniques of both ultrashort pulse shaping and pulse characterization.
\end{abstract}

PACS numbers: 42.65.Re; 42.65.Ky; 42.30.Kq

Submitted to: J. Phys. B: At. Mol. Phys.

\section{Contents}

1 Introduction 3

2 Definition of the electric field and its representation 3

2.1 Definition of the electric field . . . . . . . . . . . . . . . 3

2.2 How to represent a short pulse? . . . . . . . . . . . . . 6

3 Arbitrary Pulse shaping of ultrashort pulses $\quad 8$

3.1 Generalities . . . . . . . . . . . . . . . . 8

3.2 A spatial mask in the Fourier plane of a zero dispersion line . . . . . . 10

3.2.1 Introduction

10

3.2.2 4f-line theory in few equations

11

3.2.3 Spatio-temporal coupling

13

3.3 Most common Masks

14 
3.3.1 Liquid crystal masks

15

3.3.2 Acousto-optic modulator masks

21

3.3.3 Other masks

26

3.4 Shaping by phase transfer . . . . . . . . . . . . . . . . . . . . 27

3.4.1 Acousto-Optic programmable Dispersive Filters (AOPDF) 27

3.4.2 Non-linear frequency mixing 32

3.5 Conclusion on pulse shaping . . . . . . . . . . . . . . . . . . . . . 33

4 Characterization of ultrashort shaped pulses $\quad 36$

4.1 Prerequisite and generality . . . . . . . . . . . . 36

4.2 Incomplete characterization methods . . . . . . . . . . . . . . 37

4.2.1 Autocorrelation

37

4.2.2 Intensimetric Cross-correlation

38

4.3 Referenced complete characterization methods . . . . . . . . . . . . . 39

4.3.1 Spectral interferometry

39

4.3.2 Fourier transform spectroscopy

42

4.4 Self-referenced complete characterization methods . . . . . . . . . . . . . 43

4.4.1 Prerequisite for self-referenced measurement of $\varphi(\omega)$ 43

4.4.2 FROG and other variants

44

4.4.3 SPIDER and other variants

48

4.4.4 Conclusion on characterization

56

5 The combination of pulse shaping and characterization techniques $\quad 57$

6 General conclusion $\quad 61$

$\begin{array}{lll}7 & \text { Bibliography } & 61\end{array}$ 


\section{Introduction}

In 1966, few years after the first laser came to light, short pulses (picosecond) were available [1]. Their generation and characterization were already active fields of research. Forty-three years later, few cycle femtosecond pulses and complex shaped pulses can be synthesized in various spectral regions. These arbitrarily shaped optical waveforms are of great interest in a number of fields including coherent control $[2,3,4]$, multidimensional spectroscopy [5], biological imaging[6], compression of optical pulses[7], factorization of numbers [8, 9] and optical communications[10]. Most of these works were spurred by technological breakthroughs in arbitrary pulse shaping field [11]. These spectacular progresses have been obtained in parallel with the development of new tools for a complete characterization of ultrashort pulses $[12,13]$. We propose to describe for newcomers in the field the basics on arbitrary pulse shaping and pulse characterization. The tone of this article is to be as didactic as possible and is voluntary not a complete review. Trying to help the beginners, several tables and numerical applications enlighten the text. The authors strongly recommend interested readers to read more extensive articles such as Weiner's review [11] for pulse shaping or the recent review on characterization by Walmsley and Dorrer [13]. Finally many interesting books $[14,15,16]$ can nicely complement the present work.

This tutorial is organized as follow: section 2 gives some mathematical background for the description and representation of ultrashort pulses, section 3 is devoted to arbitrary pulse shaping, section 4 presents some of the most widespread methods for ultrashort pulse characterization and section 5 gives an overview of shaping assisted characterization.

\section{Definition of the electric field and its representation}

\subsection{Definition of the electric field}

A laser pulse is entirely defined by its electric field $\mathcal{E}(t)$. For ultrashort pulses however, $\mathcal{E}(t)$ is not easily accessed and the spectral domain is often more practical. As $\mathcal{E}(t)$ is a real function, its Fourier transform reads $\tilde{\mathcal{E}}(\omega)=1 / 2\left(\tilde{E}(\omega)+\tilde{E}^{*}(-\omega)\right)$ where $\tilde{E}(\omega)$ is the complex electric field taking non-zero values only for positive values of the frequency $\omega$.

$\tilde{E}(\omega)$ is a complex function containing all the information concerning the pulse and from which $\mathcal{E}(t)$ can be retrieved. By inverse Fourier transform of $\tilde{E}(\omega)$ one gets a complex field in the temporal domain $E(t)$, which real part is $\operatorname{Re}[E(t)]=\mathcal{E}(t)$.

$$
\mathcal{E}(t)=\int_{-\infty}^{+\infty} \tilde{\mathcal{E}}(\omega) \exp (-i \omega t) \frac{d \omega}{2 \pi}
$$

The shape of an ultrashort pulse can thus be defined equivalently by its electric field $\mathcal{E}(t)$, or by its complex electric field in the time domain $E(t)$ or in the spectral domain $\tilde{E}(\omega) . \tilde{E}(\omega)=A(\omega) \mathrm{e}^{i \varphi(\omega)}$ is usually the most practical definition and both its spectral 
amplitude $A(\omega)$ and its spectral phase $\varphi(\omega)$ contribute to the pulse shape. As an example, for a given spectral amplitude, the spectral phase alone completely determines the pulse duration and shape. The shortest duration is achieved for a flat phase and the pulse is said to be Fourier-limited. When a pulse is Fourier-limited, its duration is inversely proportional to its spectral width. When it is no longer Fourier limited, its duration increases. The time-bandwidth product (TBP) provides a way to estimate how far is a pulse from the Fourier limit. TBP is the product of time duration and spectral width of a given pulse

$$
\mathrm{TBP}=\Delta t . \Delta \omega
$$

where $\Delta t$ and $\Delta \omega$ are the full width at half maximum (FWHM) in intensity of the pulse temporal and spectral profiles $\ddagger$. Another way to estimate the distance from Fourierlimited is the complexity [11]. It can be interpreted as the ratio between the largest and the smallest features in either time or spectral domains:

$$
\eta=\Delta t / \delta t=\Delta \omega / \delta \omega
$$

as sketched on figure 1. TBP and complexity are proportional and for a gaussian

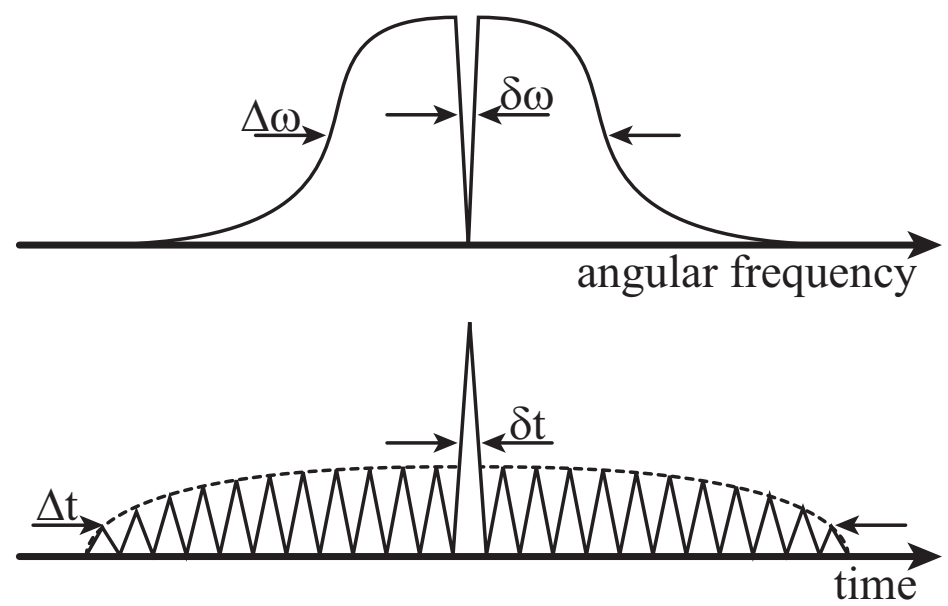

Figure 1. The complexity can be seen as the ratio between the largest and smallest features in either time or spectral domains. $\Delta t$ and $\delta t$ (resp. $\Delta \omega$ and $\delta \omega$ ) are the largest and smallest features in time (resp. in spectral) domain.

spectrum one gets:

$$
\eta \simeq \mathrm{TBP} / 4 \ln 2
$$

The spectral phase plays a central role in determining the complexity, and more generally the shape, of a short pulse. There are several quantities related to the spectral phase that are often used to describe the evolution of a shaped pulse. The group delay is defined as $T_{g}(\omega)=\partial \varphi / \partial \omega$ and it describes the arrival time of each spectral component of the pulse. The instantaneous frequency, defined as $\omega(t)=\partial \varphi_{t} / \partial t$ (where $\varphi_{t}$ is the $\ddagger$ We arbitrary choose to work with FWHM 
temporal phase), gives the opposite point of view as it describes the temporal evolution of the frequency of the short pulse. Both can be extremely useful to grasp the main characteristic of a given shaped pulse.

Expanding the spectral phase in Taylor series is another useful tool in order to analyze the effect of each term of the series:

$$
\begin{aligned}
\varphi(\omega)=\varphi_{0}^{(0)} & +\varphi_{0}^{(1)}\left(\omega-\omega_{0}\right)+\frac{1}{2} \varphi_{0}^{(2)}\left(\omega-\omega_{0}\right)^{2}+\frac{1}{6} \varphi_{0}^{(3)}\left(\omega-\omega_{0}\right)^{3} \ldots \\
& +\frac{1}{24} \varphi_{0}^{(4)}\left(\omega-\omega_{0}\right)^{4}+\ldots+\frac{1}{n !} \varphi_{0}^{(n)}\left(\omega-\omega_{0}\right)^{n}
\end{aligned}
$$

with $\varphi_{0}^{(0)}=\varphi\left(\omega_{0}\right)$ and $\varphi_{0}^{(n)}=\left(d^{n} \varphi / d \omega^{n}\right)_{\omega_{0}}$.
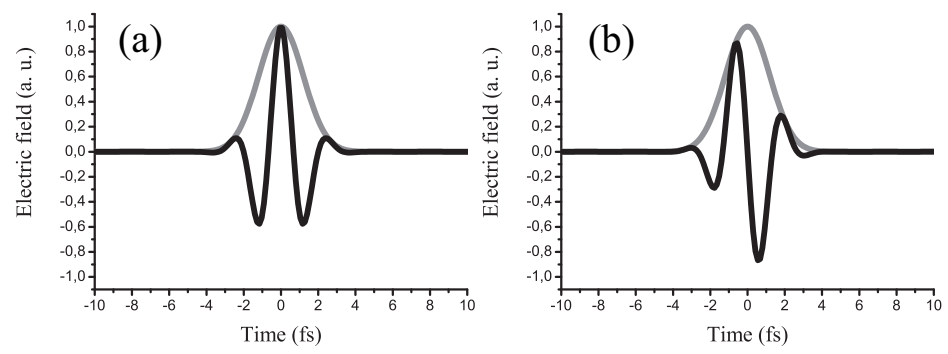

Figure 2. $\varepsilon(t)$ (in black) and envelope (in gray) of the electric field of a short laser pulse in two case of carrier envelope phase. The pulse has a central wavelength of $800 \mathrm{~nm}$ and a FWHM of 2 fs. In (a) a 0 rad CEP shows a cosine pulse and in (b) a $\pi / 2$ rad CEP shows a sine pulse.

- $\varphi_{0}^{(0)}$ is known as the absolute phase, or Carrier Envelope Phase (CEP), which corresponds to the phase between the envelope of the electric field and the carrier. This phase plays a key role in non-linear interaction as High harmonic generation when pulses are short enough so that only few cycles are under the envelope (cf figure 2).

However, in this tutorial, we consider many-cycle pulses and we do not focus on CEP. Interested readers can refer to [17] for CEP measurement and control.

- $\varphi_{0}^{(1)}$ simply corresponds to a delay between the pulse and an arbitrary origin of time. This leads to a constant group delay.

- $\varphi_{0}^{(2)}$ is the most famous term, well-known as chirp. It induces an increase of the pulse duration. Around any given frequency under the pulse spectrum, the quadratic phase can be approximated by its tangent, whose slope is simply the group delay which evolves linearly with the frequency. In other words, each frequency experiences a delay that linearly increases as we scan through the spectrum, as shown on figure 3 (b). For a strong chirp $\left(\varphi_{0}^{(2)} \gg \Delta t_{T F}^{2}\right.$, where $\Delta t_{T F}$ is the Fourier limited pulse duration), the instantaneous frequency is given by $\omega(t)=\omega_{0}+t / \varphi_{0}^{(2)}$. Many 
experiments have been performed to study the interaction of atoms or molecules with a chirped pulse leading to fascinating results [18, 19, 20, 21].

- $\varphi_{0}^{(3)}$ corresponds to the cubic phase. It leads in the temporal domain to many preor post-pulses (figure 3 (c)) and can be exploited in coherent control schemes [22]. When aiming for really short pulses, cubic phase as well as higher orders have to be properly compensated for.

It is worth noting that some phases cannot be expanded in Taylor series, such as sinusoidal phases or phase-jumps that are routinely used in coherent control schemes.

\subsection{How to represent a short pulse?}

The most common way to represent a short pulse is to plot both its spectral amplitude $A(\omega)$ and its spectral phase $\varphi(\omega)$ as shown on the first column of figure 3 for a flat phase, a $\varphi_{0}^{(2)}$, a $\varphi_{0}^{(3)}$, a $\pi$-jump and a two-pulse sequence. Another possibility is to plot the temporal intensity§ $|E(t)|^{2}$, as shown on the second column of figure 3 .

However, in some cases, time-domain or frequency-domain representations are not enough, especially when both time and frequency properties are strongly coupled. Timefrequency distributions such as Wigner function have been proposed to provide an intuitive mapping of these time-frequency couplings [23, 24]. The Wigner function of an electric field $E(t)$ can be written as [24]:

$$
W(t, \omega)=\int E\left(t+\frac{t^{\prime}}{2}\right) E^{*}\left(t-\frac{t^{\prime}}{2}\right) e^{i \omega t^{\prime}} d t^{\prime}
$$

or alternatively using its spectral electric field $\tilde{E}(\omega)$ :

$$
W(t, \omega)=\frac{1}{2 \pi} \int \tilde{E}\left(\omega+\frac{\omega^{\prime}}{2}\right) \tilde{E}^{*}\left(\omega-\frac{\omega^{\prime}}{2}\right) e^{-i \omega^{\prime} t} d \omega^{\prime}
$$

The equivalence of these two definitions can be shown using Fourier transform properties. The third column of figure 3 shows the Wigner functions for different pulse shapes. As an example, for a $\varphi_{0}^{(2)}$ (case (b)), one can clearly see the linear sweep in time of the instantaneous frequency. Albeit it contains all the information on the pulse and it usually provides an intuitive representation for complex pulse, the Wigner function can sometimes be difficult to interpret. As an example, for a two-pulse sequence (case (e)), there are fringes and a maximum around $t=0$ although there is no energy at that time in the two-pulse sequence. For the case of two pulses or cubic phase, the Wigner function can be negative. But the marginals, which represent the temporal and spectral intensity of the pulse, are always positive [13]. A more intuitive alternative to the Wigner function is the spectrogram that can be written as

$$
S(t, \omega)=\left|\int E\left(t^{\prime}\right) g\left(t^{\prime}-t\right) e^{i \omega t^{\prime}} d t^{\prime}\right|^{2}
$$

$\S$ This time representation does not carry all the information on the pulse as the oscillations under the envelope are not shown. 


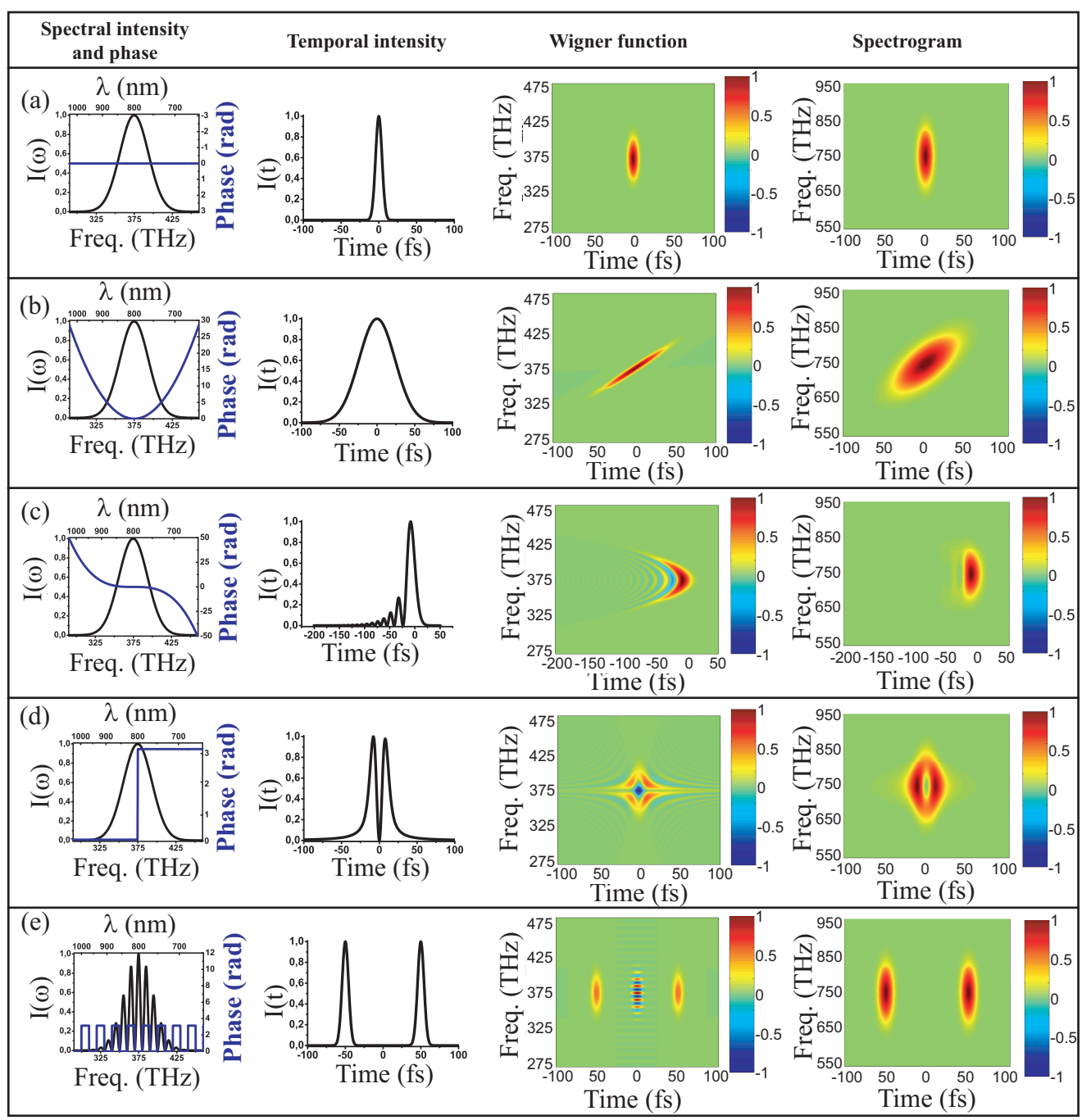

Figure 3. Pulse shaping gallery; (a) Fourier transform pulse, (b) chirped pulse, (c) cubic phase, (d) spectral $\pi$-jump, (e) two-pulse sequence. The first column presents the spectral intensity and phase, the second one, the temporal intensity, the third one the Wigner function and the last one the spectrogram. For the two latter cases, the gate chosen is a Fourier limited 10 fs-pulse at $800 \mathrm{~nm}$.

The spectrogram is the time convolution of the electric field with a gate $g(t)$ that can be the pulse itself. In the last column of the figure 3, the spectrogram of each case is plotted : the gate chosen is a Fourier limited pulse at $800 \mathrm{~nm}$ with a FWHM duration of 10 fs. Some well-known complete characterization techniques retrieve the spectrogram $[25]$. 


\section{Arbitrary Pulse shaping of ultrashort pulses}

\subsection{Generalities}

Use of optimally shaped pulses to guide and control the temporal evolution of a system has been an active field of research for the last twenty years. Two main approaches have been developed.

In the absence of predesigned control mechanisms a closed loop scheme [26, 27, 28, 29, 30, 31, 32, 33, 34] may be employed to find efficient pulse shapes : The outcome of many different shapes is fed back into an algorithm that iteratively optimizes the excitation shape without insight into the physical mechanism that is triggered by a particular shape.

In contrast the effect of shapes on model systems can be systematically studied within an open-loop scheme[35, 36, 37, 38, 39, 40]. This open-loop approach is well adapted to small systems for which theoretical predictions are reliable. It consists of reaching a specific goal (manipulation of the temporal response of a system excited by a light pulse) without any experimental feed-back. Physical analysis of the process allows predetermining the theoretical pulse shape which leads to the desired result. It is then implemented experimentally. Some results by "test-error" have also been obtained in complex systems following this open-loop approach[41].

Recently people have tried to combine both approaches to retrieve information from the complex pulses designed by the close loop-approach[42, 43]. In particular, interesting results have been obtained in the strong field domain[44, 45, 46, 47].

This field has pushed new developments, in particular to extend pulse shapers capabilities

- in term of refreshing rate, which is crucial in the closed-loop approach,

- in term of time shaping window to extend the dimension of the phase space,

- in term of spectral bandwidth to be able to control a great variety of systems...

In this section we will present the basics and some key results. We will insist on the most fundamental principles, limitations and experimental advices to allow newcomers in the field to develop and correctly use their own device.

Pulse shaping techniques presented here are based on the linear, time-invariant filter. They can be described either in the time domain or in the frequency domain.

In time domain, the output of a linear filter (cf figure 4) is simply given by the following convolution product

$$
E_{\text {out }}(t)=R(t) \otimes E_{\text {in }}(t)
$$

where $R(t)$ is the impulse response of the pulse shaper. However, for femtosecond pulses, direct shaping in time is quite a hard task and most of the devices operate in the spectral domain and the output pulse can be written :

$$
\tilde{E}_{\text {out }}(\omega)=H(\omega) \tilde{E}_{\text {in }}(\omega)
$$


(a) Frequency domain

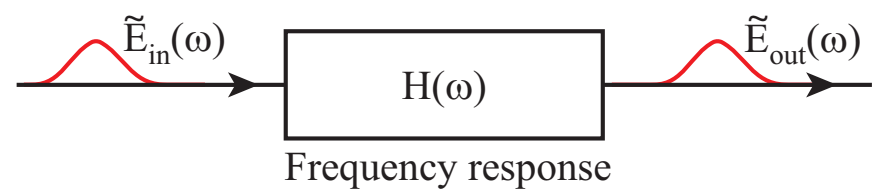

(b) Time domain

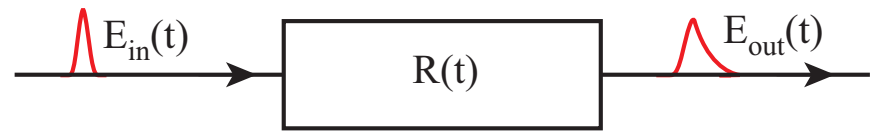

Impulse response

Figure 4. Pulse shaping by linear filtering. (a) Frequency domain. (b) Time domain.

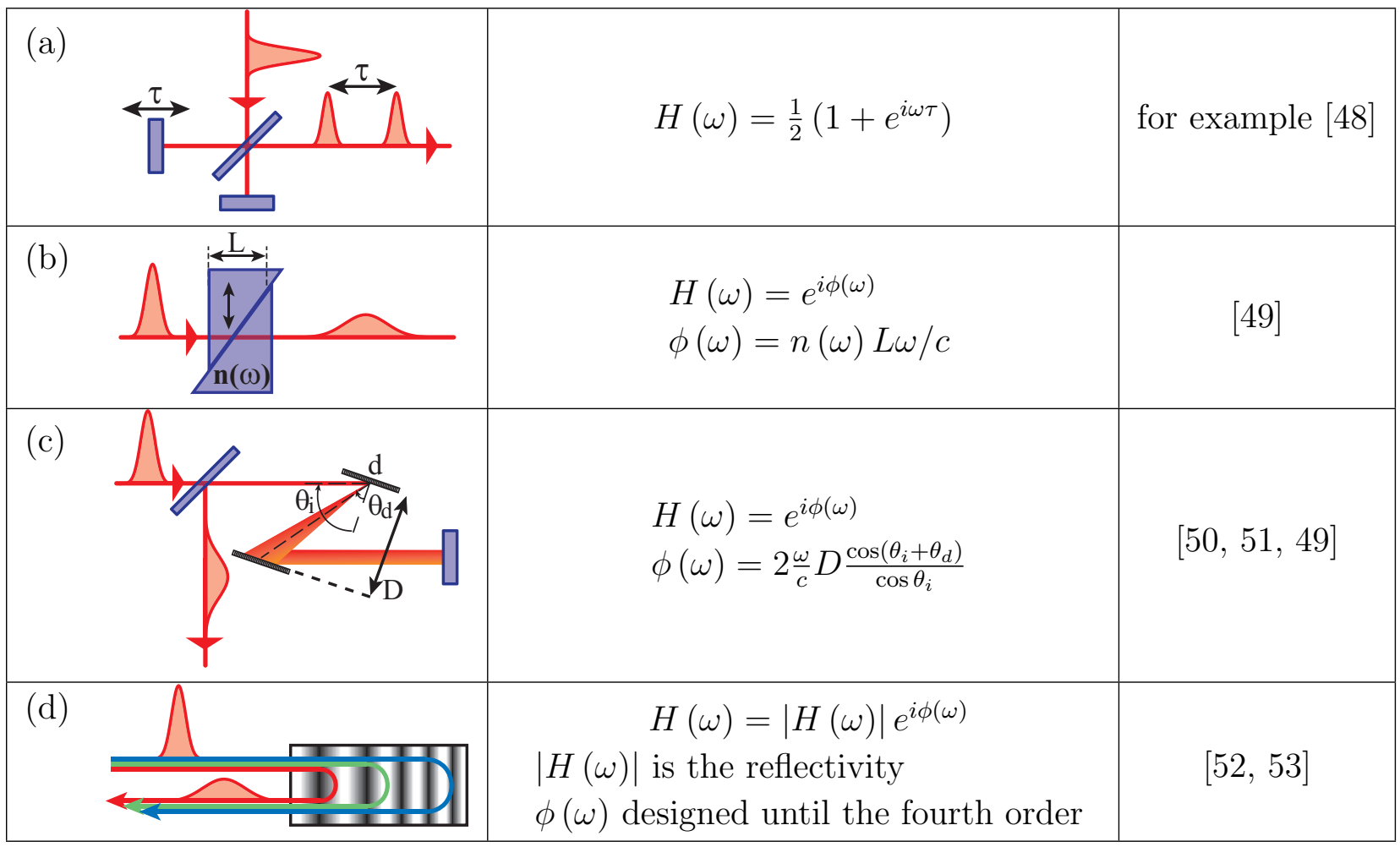

Table 1. Simple shaping setups. (a) Michelson interferometer producing two delayed pulses, (b) Propagation through a dispersive bulk medium (length L, index n), (c) grating compressor $\left(\theta_{i}\right.$ incident angle, $\theta_{d}$ diffraction angle, $\mathrm{D}$ distance between the two gratings and $d$ the interline spacing) and (d) chirped mirror (In a chirped mirror, the penetration depth depends on the wavelength which introduces chirp on a Fourier Limited pulse). The first column gives a schematic of the set-up, the second column its transfer function and the last column gives few references.

where the transfer function $H(\omega)$ completely describes the pulse shaper.

In the past decade, a lot of efforts was put in developing versatile pulse shapers based on programmable masks. Many programmable pulse shapers are now available but none is universal: they are all complex systems with extremely diverse ranges of 
usage. All currently available programmable pulse shapers address the pulse components by spatially separating them. Thus they introduce a spatio-temporal coupling (see section 3.2.3).

In order to buy or develop the right pulse shaper for a given experiment, we recommend listing its requirements in terms of : central frequency, tunability, spectral bandwidth, complexity and maximum duration of the shaped pulse, power density, repetition rate, etc... From this list one can sort the available solutions according to their adequation to the experiment specificities. Sometimes, it also points out that a programmable pulse shaper is not necessary and that the required waveforms are easily produced using usual optics. In particular if the complexity $\eta$ (see section 2.1) of the aimed shape is above few hundreds, it exceeds the possibilities of current pulse shapers, as we will see in the following. As an example, two pulses of $20 \mathrm{fs}$ separated by $60 \mathrm{ps}$ leads to a complexity of 3000! Fortunately, this is easily achievable using a Michelson interferometer. The table 1 summarizes main devices which can be more appropriate, for some specific shapes, even if they are less versatile than a programmable pulse shaper.

\subsection{A spatial mask in the Fourier plane of a zero dispersion line}

\subsubsection{Introduction}

In 1983, Froehly and coworkers [54] proposed a new design of versatile pulse shaper, the so-called zero dispersion line or 4 f-line.

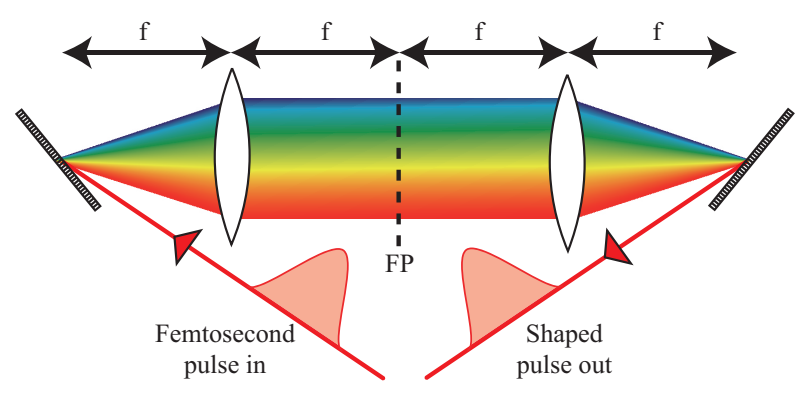

Figure 5. A zero dispersion line composed of two gratings and two lenses of focal length $\mathrm{f}$, arranged in a $4 \mathrm{f}$ set-up. The output pulse is identical to the input pulse. In the Fourier plane FP, all the spectral components are spatially separated and focused.

A 4f-line is a particular spectrometer composed of two gratings and two lenses arranged in a $4 \mathrm{f}$ set-up, where the CCD camera is replaced by a mask (cf Fig 5). Each spectral component is angularly dispersed by the first grating then focused close to small diffraction spots in the Fourier plane by the first lens/mirror. Thus, in this plane, all the spectral components are spatially separated and focused. Then a second combination of lens/grating allows recombining all the frequencies into a single collimated beam. If nothing is placed in the Fourier plane then the device is dispersion free (zero dispersion line) and the output beam is identical to the input one. By putting a specific mask in the Fourier plane, one can modify the optical path and/or optical density for each 
spectral component and thus shape the output pulse. For ultrashort pulses (broad spectral bandwidth), the lenses are often replaced by cylindrical/spherical mirrors to avoid unwanted dispersion and chromatic aberrations. Depending on the mask, a $4 \mathrm{f}$ pulse shaper controls the phase [11], both phase and amplitude [55], the polarization [56] and also the transverse spatial profile [57].

\subsubsection{4 -line theory in few equations}

We present here the basic equations that govern 4f-line abilities for shaping[58]. We consider the input pulse to be gaussian in both time and space, with a central frequency $\omega_{0}$ (corresponding to a central wavelength $\lambda_{0}$ ), and with the following Full Widths at Half Maximum -FWHM- in intensity:

- $\Delta \omega_{L}$ in the spectral domain,

- $\Delta t$ in the temporal domain\|,

- $\Delta x_{i n}$ in the spatial domain 9 .

As shown on figure 6 , the input pulse is diffracted by the first grating with an angle $\theta_{d}$ at $\lambda_{0}$ ( $\theta_{i}$ being the input angle), $d$ is the grating period and $f$ the focal length. In the Fourier plane, each spectral component has a finite size $\Delta x_{0}$ due to diffraction. It is easily derived from gaussian beam propagation:

$$
\Delta x_{0}=2 \ln (2) \frac{\cos \theta_{i}}{\cos \theta_{d}} \frac{f \lambda_{0}}{\pi \Delta x_{i n}}
$$

Let us now consider the spatial coordinate $X$ in the Fourier plane. Assuming linear dispersion, a frequency $\omega_{k}$ is located in the Fourier plane at $X_{k}$ given by:

$$
X_{k}=\alpha \omega_{k}
$$

where $\alpha$ is set by the $4 \mathrm{f}$-line geometry :

$$
\alpha=\frac{\lambda_{0}^{2} f}{2 \pi c d \cos \theta_{d}}
$$

The frequency resolution is easily obtained from equations 12 and 13 :

$$
\delta \omega=\Delta x_{0} / \alpha
$$

By Fourier transform, this corresponds to a window $T$ in the time domain:

$$
T=4 \ln (2) / \delta \omega=\Delta x_{i n} /|v|
$$

where $|v|$ has the dimension of a velocity:

$$
v=c d \cos \theta_{i} / \lambda_{0}
$$

$T$ is the time window available for shaping and equation 15 clearly states that it is proportional to the input waist. This unexpected property of the 4 f-line is a manifestation

$\|$ Time and frequency FWHM in intensity verify the following inequality: $\Delta t \Delta \omega_{L} \geq 4 \ln (2)$

II It is related to the waist $\mathrm{w}_{\text {in }}$ of the beam at the input of the $4 \mathrm{f}$-line, by $\Delta x_{i n}=\mathrm{w}_{\text {in }} \sqrt{2 \ln (2)}$ 
of spatio-temporal coupling (see section 3.2.3 for more details) and $v$ is usually called the spatio-temporal coupling velocity. $T$ defines a temporal upper bound for shaping achievable with such a $4 \mathrm{f}$-line: the duration or the time delay of the shaped pulse should stay below $T$, otherwise strong distortions will occur. The temporal lower bound is given by the inverse of the spectral bandwidth of the input pulse, which means that the shaper shortest feature is governed by the available optical bandwidth ${ }^{+}$.

\section{Numerical applications}

- In the IR [60] : gratings $: 1 / d=2000 \mathrm{gr} / \mathrm{mm} ; f=600 \mathrm{~mm}$; beam diameter $\Delta x=2 \mathrm{~mm}$, incident angle $\theta_{i}=64^{\circ}, \lambda_{0}=800 \mathrm{~nm}, \Delta \lambda_{L}=10 \mathrm{~nm}$. This leads to a temporal window $T \simeq 24$ ps and a complexity $\eta \simeq 255$.

- In the mid-IR [61] : $1 / d=300 \mathrm{gr} / \mathrm{mm} ; f=125 \mathrm{~mm}$; incident angle (Littrow configuration) $\theta_{i}=47^{\circ}, \Delta x_{0}=250 \mu \mathrm{m}, \lambda_{0}=4900 \mathrm{~nm}, \Delta t=140$ fs. This leads to a temporal window $T \simeq 78$ ps and a complexity $\eta \simeq 557$.

To turn a 4f-line into a pulse shaper, we put a mask in its Fourier plane. It has a number of control parameters which can be pixels or not. Then to maximize pulse shaping capability, one should fulfill the following constraint :

$$
\text { control parameter number } \geq \eta
$$

This mask will act both as a spatial mask $M_{X}$ and a spectral mask $M_{\omega}$. Immediately

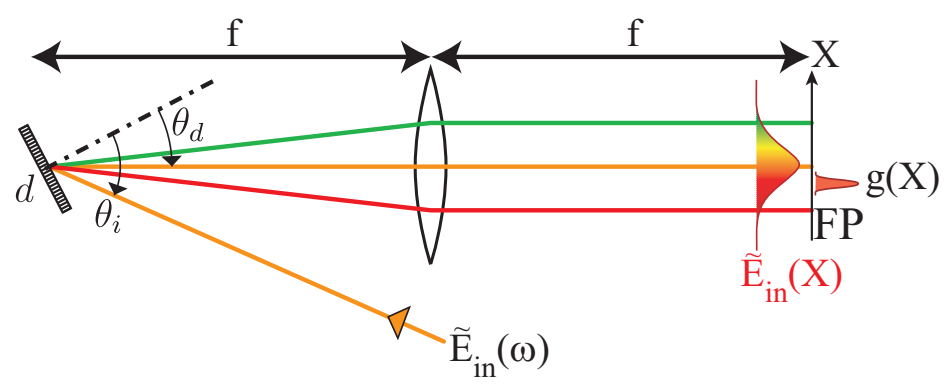

Figure 6. Half a 4f-line : The Fourier plane is situated in the back focal plane of the lens. $f$ is the focal length. $X$ is the spatial coordinate in this plane. $g(X)$ is the spatial extension of a given frequency component. $\theta_{i}$ is the incident angle on the grating. $d$ is the grating period. $\theta_{d}$ the diffraction angle.

after the mask, the pulse can be written as :

$$
\tilde{E}_{a f t e r}(\omega, X)=\tilde{E}_{i n}(\omega) \cdot g(X-\alpha \omega) M_{X}(X)
$$

where

$$
g(X)=\exp \left[-2 \ln (2)\left(X / \Delta x_{0}\right)^{2}\right]
$$

+ It is possible to obtain shorter pulse than Fourier limited one by shaping the spectral amplitude. The cost is the appearance of a pedestal in the time domain as well as a decrease of energy [59] 
is the spatial extension of a given frequency component. As position and frequency are coupled in the Fourier plane, both the spectral and spatial components of the pulse are affected by the mask. As a result, propagating the pulse through the second half of the pulse shaper requires special care and goes beyond simple gaussian propagation. Letting this aside using gaussian propagation, gives a linear expression for the output pulse $\tilde{E}_{\text {out }}(\omega)$ :

$$
\tilde{E}_{\text {out }}(\omega)=H(\omega) \tilde{E}_{\text {in }}(\omega)
$$

where

$$
H(\omega)=\int M_{\omega^{\prime}} g\left(\omega^{\prime}-\omega\right) d \omega^{\prime}=M_{\omega} \otimes g(\omega)
$$

and $M_{\omega}(\omega)=M_{X}(\alpha \omega)$. To obtain this expression, we have implicitly assumed that the spatial profile is unaffected by the mask, which is a strong simplification. As a consequence, this expression is only valid for sufficiently smooth and simple shapes and for the on-axis part of the pulse (that is what is left after a subsequent spatial filtering).

An illustration of the effect of the time window $T$ is given on figure 7 (c) that shows the intensity trace of a pulse delayed using a $4 \mathrm{f}$ pulse shaper. As the programmed delay moves away from 0 , the pulse peak intensity decreases and follows a gaussian envelope of FWHM in intensity of around $24 \mathrm{ps,} \mathrm{which} \mathrm{corresponds} \mathrm{to} \mathrm{the} \mathrm{temporal} \mathrm{window} \mathrm{T}$ [60].

\subsubsection{Spatio-temporal coupling}

Let us describe more precisely the way the $4 \mathrm{f}$-line acts on the beam. The first grating is coupling each frequency $\omega$ into a given direction $k_{\omega}$ and the first lens (or mirror) is mapping $k_{\omega}$ to a given position $X_{\omega}$ in the Fourier plane. As a result, the position $X_{\omega}$ in the Fourier plane corresponds both to a certain spectral component and to a certain direction (i.e. a certain spatial frequency). The part of the mask lying at $X_{\omega}$ thus acts simultaneously on both $\omega$ and $k_{\omega}$ and this is the origin of the spatio-temporal coupling. After going through the second half of the 4 f-line, all the components are back together. At the output of the pulse shaper, the net result of spatio-temporal coupling is that the transverse profile of the beam is modulated accordingly to the temporal pulse shape of the pulse.

For example in the case of a delay, the mask is a linear phase applied to both frequencies and spatial frequencies. The shaped pulse is thus shifted in time, but also shifted along the transverse coordinate. This is illustrated on figure 7 (a) where we have plotted the pulse profile versus time (horizontal axis) and space (vertical axis) for various programmed delays: as the delay is changed, the pulse appears to move along a slanted line (black dash) which slope is precisely the spatiotemporal coupling speed $v$. Several articles $[62,63,64]$, including recent ones $[65,66]$ have covered spatio-temporal coupling in details.

Spatio-temporal coupling is inherent to all programmable pulse shapers and in particular $4 \mathrm{f}$ line pulse shapers and cannot be avoided, in particular when asking for 

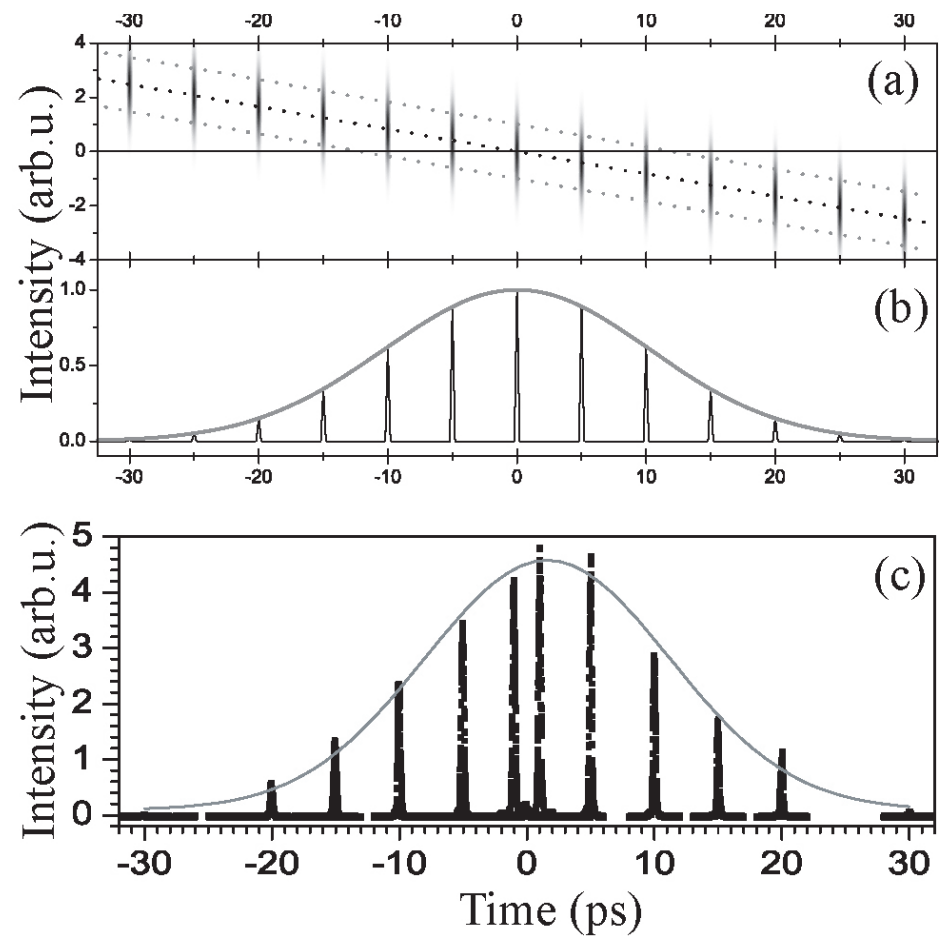

Figure 7. Space-time coupling in the case of a delay: (a) Theoretical plot versus time and transverse position of a shaped pulse for various programmed delays. (b) Cross-section of plot (a) along the axis of propagation (transverse position $=0$ ). (c) Experimental cross-correlations of shaped pulses for various delays.

complex waveforms or when pushing a $4 \mathrm{f}$ pulse shaper to its limits. One simple test to determine if spatio-temporal coupling is negligible is if one is able to shape the phase without affecting the amplitude of the pulse (a $\pi$-step spectral phase could be a good test has demonstrated in [64]). However, its effect can be reduced by designing a 4f-line with a spatio-temporal coupling speed $v$ as small as possible. Impressive results have been obtained in open-loop coherent control approach which clearly demonstrate that this effect is not detrimental for these experiments[38, 36].

\subsection{Most common Masks}

Nowadays different kind of masks can be placed in the Fourier plane of a $4 \mathrm{f}$-line to form a pulse shaper. We propose to describe more in details the liquid crystal and the acousto-optic modulator masks, which are the most representative in the field. Some others are also available and will be briefly mentioned afterwards. For all these masks, one important parameter is the total refreshing time which is the time required to obtain a given programmable waveform. This time includes the loading time depending on the data transfer mode (USB, GPIB, etc...) and the physical refreshing time depending on the mask itself ( for example, orientation of the liquid crystal, travelling time of the acoustic wave, movements of the actuators...). 


\subsubsection{Liquid crystal masks}

Each pixel of a Liquid Crystal Spatial Light Modulator (LC-SLM) is a programmable waveplate controlled by voltage. LC-SLM consists of a thin layer of nematic liquid crystal placed between two glass substrates. One substrate is covered with transparent ITO (Indium Tin Oxide) electrodes which allow applying a particular voltage independently to each pixel. The nematic liquid crystals are small rods which are oriented parallel to the substrate when no voltage is applied. Their anchorage direction is fixed by brushing of the electrodes. When a voltage is applied, the nematic tend to align along the field. This modifies the birefringence of the medium, leading to a modification of the optical path for light polarized along the anchorage direction. For a frequency $\omega$, the pixel acts as a waveplate which phase $\phi$ is given by

$$
\phi(\omega, U)=\frac{\omega \Delta n(\omega, U) e_{C L}}{c}
$$

where $\Delta n(\omega, U)$ is the index difference between slow and fast axis, $U$ the applied voltage, and $e_{C L}$ the liquid crystal thickness. The pixels have a typical width of $97 \mu \mathrm{m}$ with a gap of $3 \mu \mathrm{m}$ (cf figure $8(\mathrm{a})$ ). This gap-zone is not really controlled and will lead to undesirable effects (see below).

To achieve amplitude and phase shaping, two spatial light modulators are needed with anchorage direction at $+45^{\circ}$ and $-45^{\circ}$ with respect to the horizontal axis. By placing horizontal polarizers at both the input and output of the $4 \mathrm{f}$-line, the complex transfer function of one particular pixel $k$ can be written as

$$
H_{k}=\exp \left(i \frac{\phi_{1}\left(\omega_{k}\right)+\phi_{2}\left(\omega_{k}\right)}{2}\right) \cos \left(\frac{\phi_{1}\left(\omega_{k}\right)-\phi_{2}\left(\omega_{k}\right)}{2}\right)
$$

where $\omega_{k}$ is the frequency impinging on pixel $k . \phi_{1}$ and $\phi_{2}$ correspond respectively to the phase introduced by the first and second LC-SLM. With the same set-up, it is also possible to shape phase and polarization, by removing all the polarizers [56]. In that case, however one should keep in mind that most of the usual optics (mirrors, beamsplitter, gratings, ...) are polarizing elements that can alter the waveform.

\section{Alignment and calibration}

Performances and accuracy of the pulse shaper are strongly correlated to the quality of the alignment and the calibration. At least three steps are necessary to obtain a reliable pulse shaper that can be used in open-loop. We will describe these steps for a 4f-line fitted with two LC-SLM (to shape both phase and amplitude).

- The first step consists on a careful alignment of the 4 f-line. This step depends on the chosen geometry and optical components. Several geometries with varying degrees of compacity and optical aberrations [67, 68, 69, 70, 60] have been used. We will focus on the folded geometry [60] as depicted on figure 8(b) as it is easy to align and almost aberration-free. In this line, distances are such that the grating is in the front focal plane of the cylindrical mirror and the folding mirror in its back 


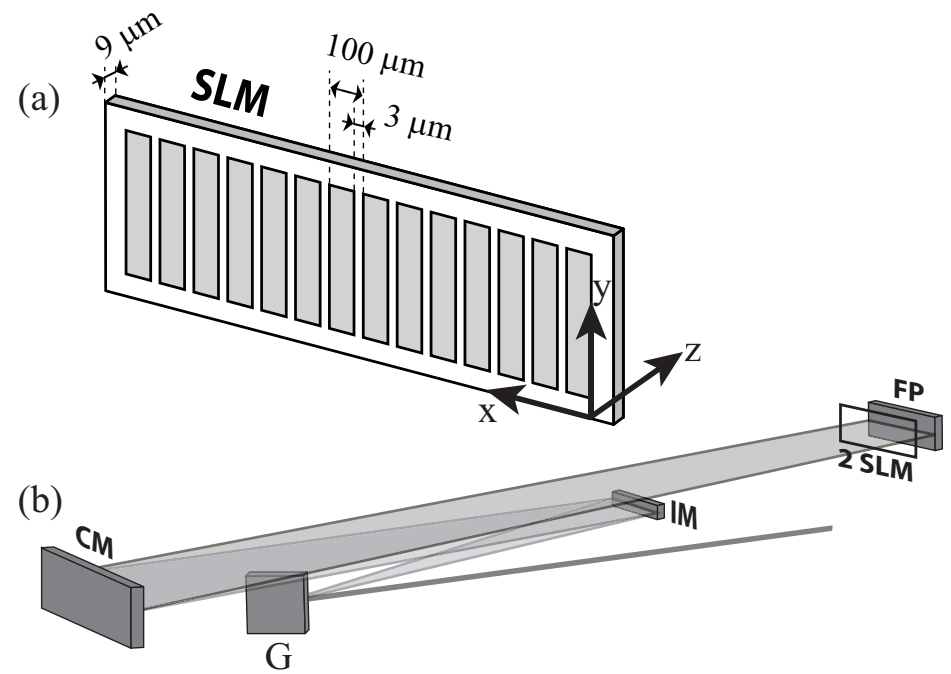

Figure 8. (a) 640 pixels Liquid Crystals spatial light modulator. (b) A folded zero dispersion line geometry with almost no optical aberration. G : grating; IM : intermediate mirror; CM: cylindrical mirror; FM: folding mirror; SLM : liquid crystal mask.

focal plane. The input beam is first diffracted by the grating $G$, then sent to the cylindrical mirror $\mathrm{CM}$ via an intermediate flat mirror IM. The cylindrical mirror focuses each spectral component to a given transverse position in the Fourier Plane. A folding mirror FM is placed in this plane and sends everything back with a small vertical tilt. The mask is situated as close as possible to the Fourier plane [63] (it should stay within the Rayleigh range). The final step for the alignment is to check that the device is really a zero dispersion line by measuring both input and output pulses duration (Autocorrelation or a self referenced technique can be used as mentioned in section 4 on characterization techniques).

- The second step is to calibrate the introduced phase $\phi(\omega, U)$ as a function of both voltage and frequency, for each LC-SLM. In principle, each pixel should be calibrated independently and at the specific frequency that impinges on it. In practice however, things are way simpler. First, the LC-SLM is usually homogeneous enough to use a unique voltage calibration for all the pixels. Second, from the voltage calibration at one specific frequency $\omega_{c a l}$, we can derive the voltage calibration for any frequency:

$$
\phi(\omega, U)=\phi\left(\omega_{c a l}, U\right) \cdot \frac{\omega}{\omega_{c a l}} \cdot \frac{\Delta n(\omega, 0)}{\Delta n\left(\omega_{c a l}, 0\right)}
$$

A simple way to calibrate the phase is to place the LC-SLM between two horizontal polarizers, illuminate with a monochromatic laser at $\omega_{\text {cal }}$ and measure the transmitted intensity $I_{c a l}$ as a function of the voltage $U$ applied to all the pixels of the mask* From equation 23 , we get that $I_{\text {cal }}(U) \propto\left[\cos \left(\phi\left(\omega_{\text {cal }}, U\right) / 2\right)\right]^{2}$. In

\footnotetext{
* In the case of a dual LC-SLM, each LC-SLM is calibrated independently, while the second one is
} 
theory, it is easy to invert this relation and retrieve $\phi\left(\omega_{c a l}, U\right)$. In practice however, problems arise at each extrema of $I_{c a l}(U)$ where the retrieved phase gets noisy and highly distorted. To overcome this difficulty, a waveplate is added next to the LC-SLM to introduce a variable phase $\phi_{w p}$ so that the transmitted intensity now reads:

$$
I_{c a l}\left(U, \phi_{w p}\right) \propto\left[\cos \left(\phi\left(\omega_{c a l}, U\right) / 2+\phi_{w p} / 2\right)\right]^{2}
$$

By repeating the voltage calibration for various $\phi_{w p}$, we get a set of transmission curves with shifted extrema as shown in figure 9, top curves. By combining the retrieved phase from each curve we obtain a phase-voltage calibration that is distortion-free across the whole voltage range as shown on the bottom curve of figure 9 .

- The last step is to calibrate the dispersion of the pulse shaper, i.e. the mapping $\left(X_{k}, \omega_{k}\right)$. The non-linear dispersion law is known and can be written as:

$$
X\left(\omega_{k}\right)-X_{0}=f \cdot \tan \left[\arcsin \left(\frac{2 \pi c}{\omega_{k} d}-\sin \left(\theta_{i}\right)\right)-\arcsin \left(\frac{2 \pi c}{\omega_{0} d}-\sin \left(\theta_{i}\right)\right)\right]
$$

where $X_{k}$ is the position of the $\mathrm{k}^{\text {th }}$ pixel, $\omega_{k}$ the frequency on the pixel $k, X_{0}$ the position of the central frequency, $\omega_{0}$ the central frequency, $f$ the focal length, $d$ the grating period, and $\theta_{i}$ the incident angle. The adjusted parameters in the experiment are $X_{0}$ and $\theta_{i}$. Practically, we measure the spectrum of the output beam using a well-calibrated spectrometer with at least the same spectral resolution than the 4f-line. To pair the frequency $\omega_{k}$ with the pixel $k$, we program a zero transmission at the pixel $k$ or a $\pi$-jump between the pixel $k$ and $k+1$. This leads to a hole in the spectrum easy to spot. Knowing the dispersion law (equation $26)$, ten points across the spectrum are sufficient to precisely calibrate the spatial dispersion in the Fourier plane. This calibration is required each time the alignment of the $4 \mathrm{f}$-line is modified.

Results

The LC-SLMs have been among the first arbitrary masks used in a 4f-line. They have been widely and successfully used in a broad domain as pulse compression[71], coherent control with high/low repetition rate laser[33, 34, 3, 35, 36, 37, 38], biological imaging [6], in quantum optics [40] and recently to cool molecules[72] but also to control ablation processes[73].

Thanks to their wide transparency window, they can be used on a broad spectral range from the IR to the UV (cf Table 2). They have been carefully studied by several groups and used to do spectral phase shaping, as well as phase and amplitude [11] or polarization shaping[56]. They are commercially available and sold by several companies leading to a variety in term of geometrical aperture, number of pixels, refreshing rate, anti-reflection coatings. The Table 2 outlines some of the available devices, given switched off. 


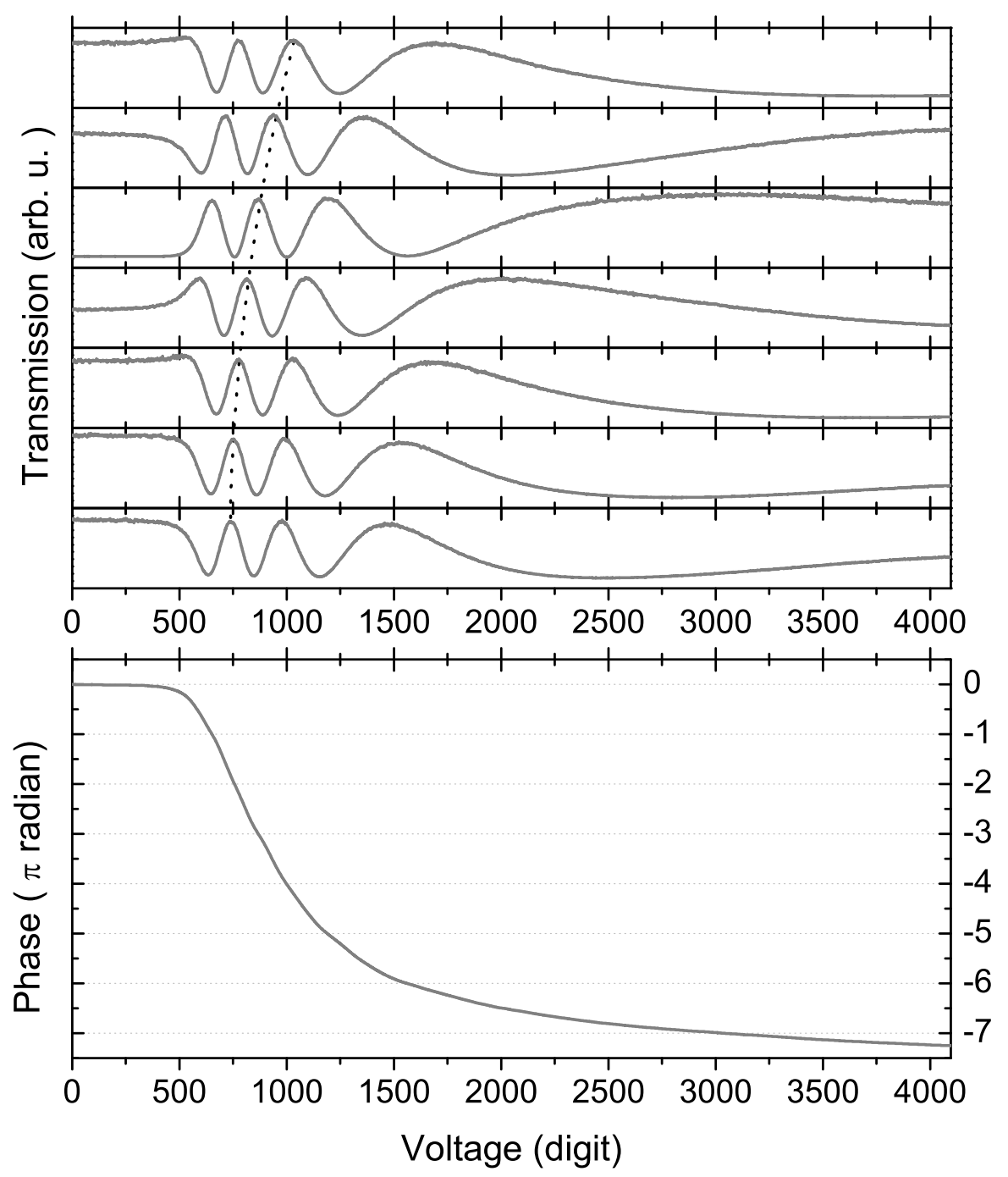

Figure 9. Phase-voltage calibration at $795 \mathrm{~nm}$ of a CRI-128 LC-SLM: Top curves: transmitted intensity as a function of the applied voltage, for several orientation of the waveplate; the position of the extrema depends on the extra phase added by the waveplate (dashed line is a guide to the eye). Bottom curve: Reconstructed phase as a function of the voltage.

some technical characteristics as the number of pixels, the type of control, the damage threshold....

To illustrate the abilities of LC-SLM 4f-line, the figure 10 shows experimental data obtained with our high resolution pulse shaper [60]. It is composed of one pair each of reflective gratings (with $2000 \mathrm{gr} / \mathrm{mm}$ ) and cylindrical mirrors $(f=600 \mathrm{~mm}$ ). Its active elements - two 640 pixels LC-SLM from Jenoptik - are installed in the Fourier plane. This provides a high resolution of $0.06 \mathrm{~nm} /$ pixel [60], with a typical $24 \mathrm{ps}$ time window. The shortest temporal feature obtained in this experiment is $94 \mathrm{fs}$. On figure 10, three examples are presented: (a) three equal amplitude pulse pairs with relative intensity $1 / 2: 1: 1 / 3$, (b) a square pulse of $700 \mathrm{fs}$, and (c) three pulses with cubic, flat, and 


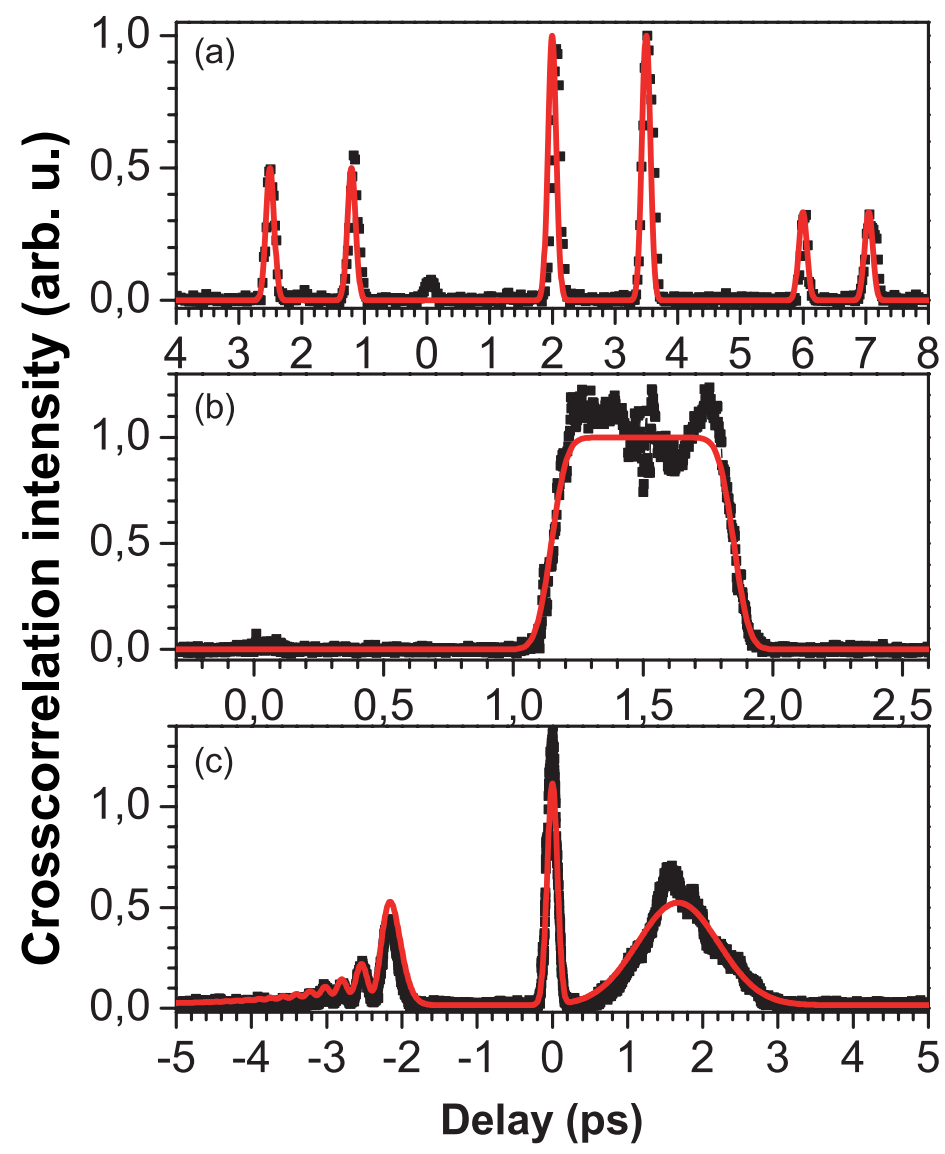

Figure 10. Three experimental waveforms(black dots) are presented: (a) three equal amplitude pulses pairs with relative intensity 1/2:1:1/3, (b) a square pulse of $700 \mathrm{fs}$, and (c) three pulses with cubic, flat and quadratic phase. The solid red line is the analytical desired wave form intensity.

quadratic phase. For each example, black dots correspond to the experimental result whereas the solid red line is the analytical desired wave form. The effect of gaps and spatial profile (gaussian temporal envelope) is almost compensated for in the applied mask.

\section{Limitations}

As mentioned above, inside the gaps of a pixelated LC-SLM, the phase is not controlled. In a first approximation the LC's behave as though there were no applied voltage. All the gaps thus have the same transmission and a small fraction of the whole spectrum is transmitted through the gaps, leading in the time domain to a small replica of the input beam at $t=0$, clearly visible in figure 11(a), where a chirped pulse has been programmed. It is possible to compensate for this gap replica by programming a replica of similar intensity but in opposite phase: they will cancel each other by destructive interferences. As the behavior in the gaps is not controlled, an iterative procedure is often needed [63] to find the right compensation replica.

The second main limitation is the pixellisation. Pixels are regular discrete elements. 

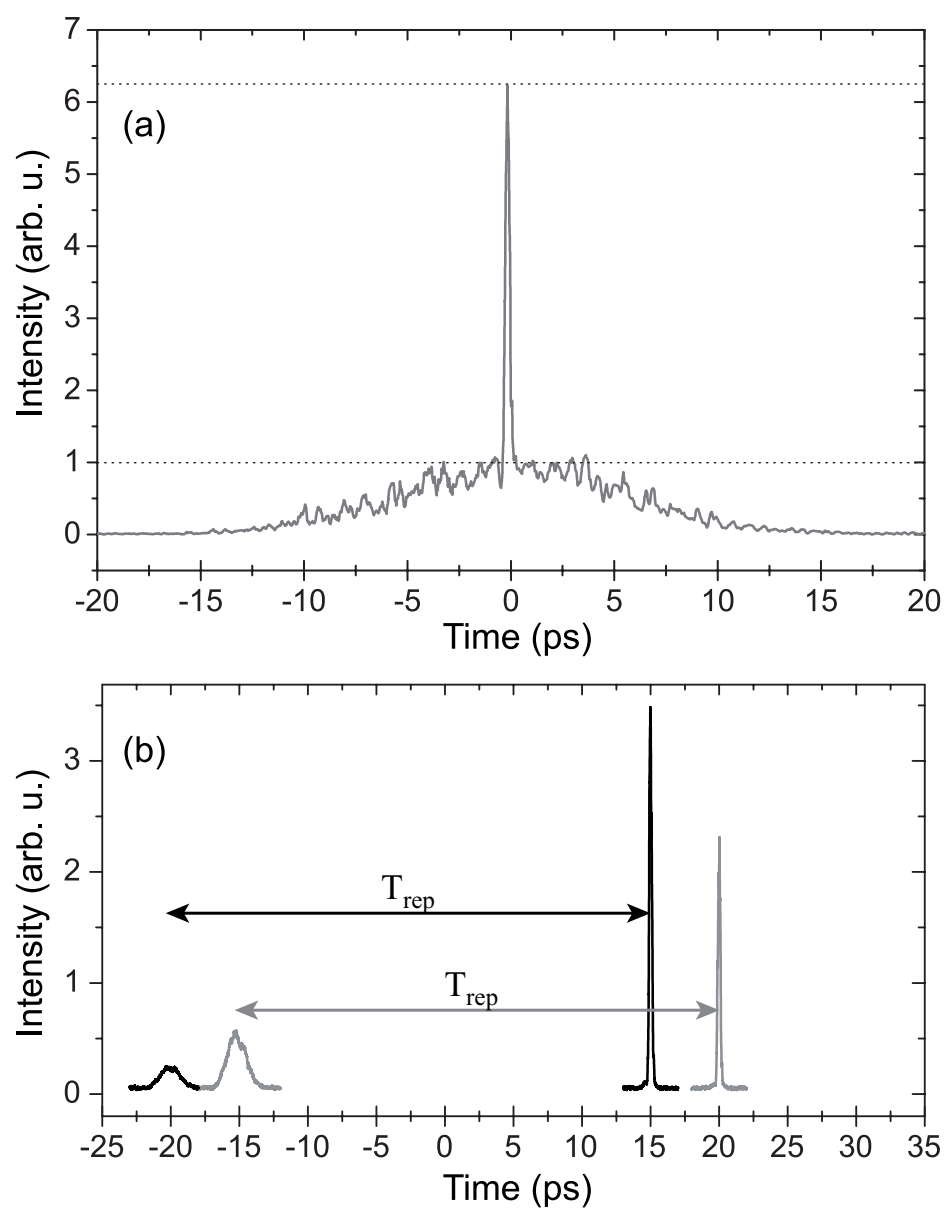

Figure 11. (a) Cross-correlation of a chirped pulse with a reference ultrashort pulse. The gap-replica is clearly spotted. (b) Cross-correlation of two delayed pulses : $+15 \mathrm{ps}$ ( black solid line) and $+20 \mathrm{ps}$ (gray solid line); Replicas due to the pixellisation of the mask are clearly seen respectively around $-20 \mathrm{ps}$ and $-15 \mathrm{ps}$. Their temporal broadening is due to the non-linear dispersion in the Fourier plane. $T_{r e p}$ is the period of the replicas, given by the spectral pixel size.

Their width in the spectral domain is denoted $\delta \omega$. The mask thus induces a discretized transfer function in the frequency domain. By Fourier transform, the impulse response is a periodic function [74] with a period $T_{r e p}=2 \pi / \delta \omega$. The figure $11(\mathrm{~b})$ presents two examples of delayed pulses which have been programmed at $\simeq 15 \mathrm{ps}$ and $\simeq 20 \mathrm{ps}$ using our high resolution pulse shaper[60]. For each one a chirped replica appears respectively at $\simeq-20 \mathrm{ps}$ and $\simeq-15 \mathrm{ps}$, which correspond to the period $T_{\text {rep }} \approx 35 \mathrm{ps}$. This value is in agreement with the pixel size in the spectral domain $\delta \lambda=0.06 \mathrm{~nm}$ (i.e. $\delta \omega \approx 0.177 \mathrm{rad} / \mathrm{ps}$ ). The replica is chirped due to the non-linear dispersion in the Fourier plane. However the gaussian temporal window (due to the spatial profile in the Fourier plane) will attenuate the replicas situated close to the edge of the window.

To exploit the spatial resolution of the mask, the incident frequency components should be focused to a spot size comparable with or less than the pixel width. If the spot size is too small, replica waveforms that arise from discrete Fourier sampling will 
not be attenuated. If the spot size is too big, the blurring of the mask will give rise to substantial diffraction effects [63].

Finally, concerning the physical refreshing time, as mentioned above, it is defined by the time required by the liquid crystal to be oriented (hundreds of $\mu \mathrm{sec}$ ). Contrary to acousto-optic devices that rely on a travelling wave (see next section), once oriented, the LC stay still and the waveform is stationary. This means that the physical refreshing time doesn't limit the repetition rate of the laser. LC are often used with oscillator sources.

\subsubsection{Acousto-optic modulator masks}

Use of acousto-optic modulator (AOM) as a SLM has been developed by Warren and coworkers $[75,76]$ in 1994. The spatial mask pattern is driven by a temporal radiofrequency voltage signal, converted into a traveling acoustic wave by a piezoelectric transducer [77]. Scaled into space by the acoustic velocity $\left(v_{a c}\right)$ in the AOM crystal, it acts as a refractive index grating. The grating period is given by the local wavelength of the acoustic waveform $\left(\Lambda=v_{a c} / \nu\right)$ where $\nu$ is the RF drive frequency. This waveform can be simultaneously phase, amplitude and frequency modulated through appropriate RF synthesis. Each spatially dispersed frequency component is then diffracted (see figure 12) with the appropriate phase and amplitude which leads to the desired Fourier transform pulse shaping.

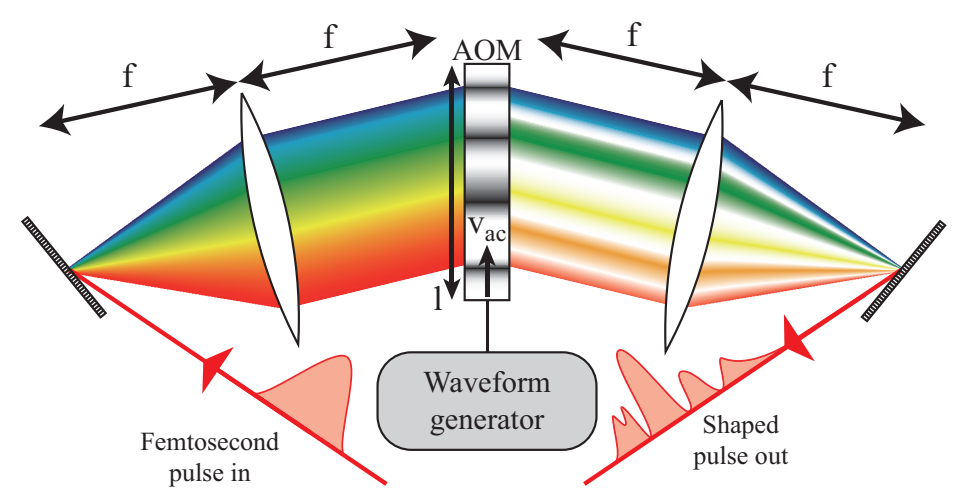

Figure 12. Programmable pulse shaper based on the use of a 4f-line and an AcoustoOptic Modulator. A radio frequency wave is generated and converted to an acoustic wave by a piezoelectric modulator. This traveling wave acts as a diffractive grating mask. $v_{a c}$ is the acoustic wave velocity, $l$ is the aperture of the AOM.

The acousto-optic effect is based on a parametric interaction [77]. As in parametric effect, a phase matching condition has to be fulfilled. The efficiency of diffraction in different orders is given by the phase mismatch $\delta_{m}$ between the diffracted wave in the $m^{t h}$ order and the free acoustic and optical waves:

$$
\delta_{m} \propto \Delta k_{m} L \propto Q\left(m-\frac{\sin \theta_{0}}{\sin \theta_{B}}\right)
$$


with the Bragg angle given by :

$$
\sin \theta_{B}=\frac{\lambda_{0}}{2 n \Lambda}
$$

This angle maximized the first order diffraction. $n$ is the refractive index of the Lthick-crystal, $\lambda_{0}$ is the optical wavelength, $\Lambda$ is the acoustic wavelength and $\theta_{0}$ the incident angle of the optical wave on the acousto-optical modulator. $Q$ is a dimensionless parameter, usually used to classified AOM regimes. It is defined by :

$$
Q=2 \pi \frac{L}{L_{e f f}}=\frac{2 \pi \lambda_{0} L}{n \Lambda^{2}}
$$

where $L_{e f f}$ is an effective length.

- In the Raman-Nath regime, the AOM is described as a thin grating $L<<L_{\text {eff }}$ which corresponds to $Q \leq 1$. This leads to a good phase matching for the different orders as can be seen on equation 27. Moreover, it has been shown that the efficiency scales quadratically with acoustic amplitude [76]. This regime allows the best spatial resolution but a limited efficiency around $30 \%$.

- For $Q \geq 4 \pi$, which means $L>>L_{e f f}$, the AOM works in the Bragg regime. Once again, regarding equation 27, the mismatch will increases dramatically with the order of diffraction. Phase matching for the first order is obtained at Bragg angle. In this regime, the efficiency could in principle reach $90 \%$.

A good compromise between efficiency and resolution for pulse shaping application appears to be for $Q \simeq 4 \pi[76]$.

\section{Alignment and calibration}

The first step to use the shaper consists on a careful alignment of the 4f-line as for LC-SLM. Then the AOM is placed in the Fourier plane and tilted until it reaches the Bragg incidence angle. For $\mathrm{TeO}_{2}\left(\lambda_{0}=780 \mathrm{~nm}, n=2.26, v_{a c}=4,2.10^{3} \mathrm{~m} / \mathrm{s}\right.$ and $\nu=200 \mathrm{MHz}$ ), this gives $\theta_{B}=0.47^{\circ}$ inside the crystal corresponding to $1.1^{\circ}$ in air [76]. The second grating should be moved to compensate for the diffraction. The set-up, shown in figure 13, is particularly well suited for AOM masks [61, 78]. Its advantage is the presence of two folding mirrors at each side of the AOM, which allows an easy compensation of the tilt induced by the AOM.

The next step is to calibrate the RF time versus the optical frequency and leads to a mask function $M(\omega)=f\left(t_{R F}=\alpha \omega\right)$. Ref [79] details the procedure of this experimental calibration. An experimental trace is shown on figure 14 .

Results

First let us briefly describe the main operating characteristics of an AOM (a complete overview can be found in [76]).

- The refreshing rate is limited by the travelling time of the acoustic wave across the modulator aperture $l$ (figure 12). For $\mathrm{TeO}_{2}$ (typical AOM crystal used in 


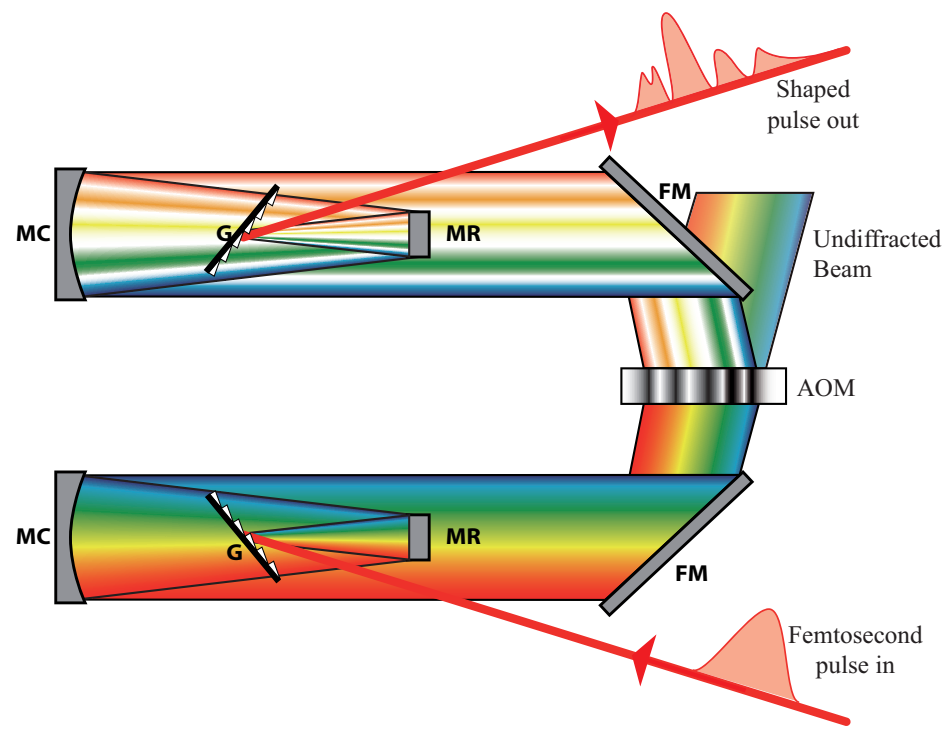

Figure 13. Experimental set-up of a 4f-line with an AOM spatial mask. A first alignment of the line is done without the AOM. In a second step, the folded mirrors (FM) are used to compensate for the AOM deflection. The first one is used to enter the AOM at Bragg angle whereas the second is used to compensate for the diffraction angle.

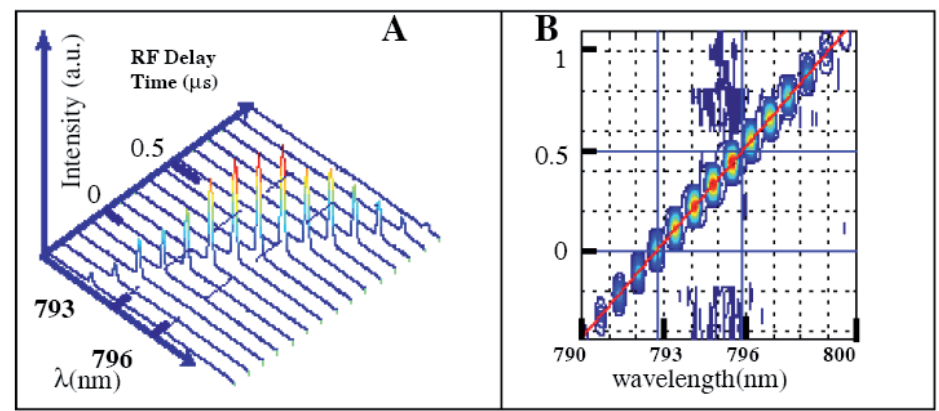

Figure 14. Taken from ref [79]. Calibration curves for AOM pulse shaper. A: each line represents a shaped spectrum created with a short RF pulse delayed in time in the AOM. Each delay corresponds to a different spatial position on the AOM leading to a different spectral position. This figure is used to calibrate the pulse shaper response function with respect to amplitude and time. B: data from A viewed as a contour plot. The solid red line fits the data, the coordinates are then used to calibrate the RF time with respect to optical wavelength.

the visible and the NIR), the acoustic velocity is $v_{a c}=4.2 \mathrm{~mm} / \mu \mathrm{s}$. In [76], the modulator has a $4.3 \mathrm{~cm}$ aperture, which leads to a corresponding time aperture $\Delta \tau=l / v_{a c}=10 \mu \mathrm{s}$. With such parameters, the diffraction pattern can then be updated on the microsecond time scale.

- The shortest resolution available is now given by the shortest acoustic feature size $\delta x$, which can be compared to a pixel in LC-SLM. The number of control parameters

\# This time is, for the acoustic device, the physical refreshing time defined above. 
is given by an effective number of pixels :

$$
N_{p i x}^{e f f}=\frac{1}{\delta x}=\Delta \tau \Delta f
$$

where $\Delta f$ is the modulation bandwidth of the AOM, 1 is the AOM aperture. It leads to approximately 900 effective pixels in ref [76]. The real resolution of the shaper is given by the combination of $N_{p i x}^{e f f}$ and $4 \mathrm{f}$-line properties described above. The typical complexity already obtained experimentally is in the order of few hundreds (around 200 for ref. [78] and 500 for ref. [61]).

Several groups have used this mask particularly well suited for optimal control $[80,81,82,83,84]$ : the refreshing rate can be as high as $100 \mathrm{kHz}$ for typical AOM-shaper. It has also been used for pulse compression [78, 80, 85] as well as coherent control $[86,87]$ and for multidimensionnal spectroscopy $[88,85,5]$. Several materials with acousto-optic properties are commercially available which allows exploring a very broad spectral range from the UV using fused silica[78, 89] to the mid IR using germanium[61]. This latter is really a challenge while it allows implementing coherent control scheme on molecular vibrational transitions excitations[84]. In this spectral range, it is probably the best technique for pulse shaping.

Figure 15[76], shows an example of complex phase and amplitude shaping. The source is a femtosecond oscillator with $82 \mathrm{MHz}$ repetition rate, a pulse picker reduces it to $20 \mathrm{kHz}$ (The acoustic refreshing rate is in the order of $100 \mathrm{kHz}$.). The waveform consists of an initial Fourier-Limited pulse followed in time by a replica, a linearly chirped pulse with a quadratic spectral phase, a cubic spectral phase pulse and finally a quartic spectral phase pulse.

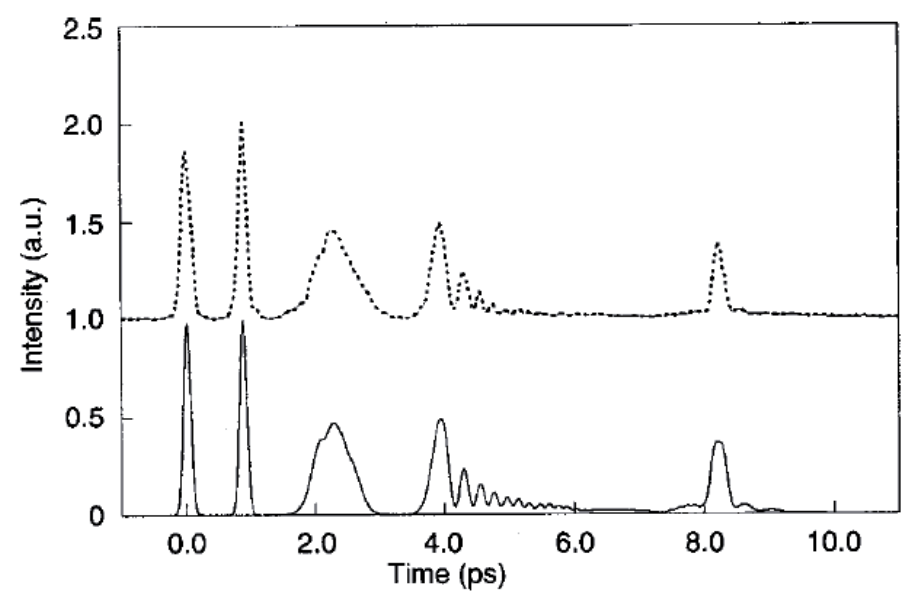

Figure 15. Taken from ref [76]. Dashed line : intensity cross-correlation trace of a shaped femtosecond pulse using AOM-SLM. The waveform used consists of an initial Fourier-Limited pulse followed in time by pulses shaped with linear, quadratic, cubic and quartic spectral phase. Solid line : theoretical trace.

An other example in mid-IR is shown in figure 16[61]. It consists of a linearly chirped pulse at $4.9 \mu \mathrm{m}$ and its corresponding Wigner function. 


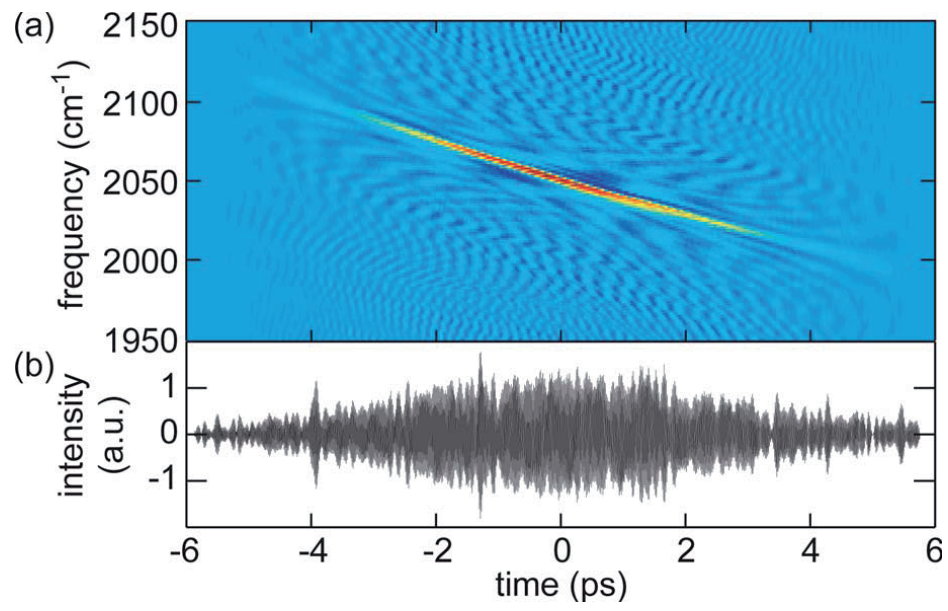

Figure 16. Taken from ref [61]. (a) Wigner diagram obtained from (b). (b) Crosscorrelation of a linearly chirped shaped pulse at $4.87 \mu \mathrm{m}$ or $2050 \mathrm{~cm}^{-1}$ in the mid IR.

\section{Limitations}

The non-linear geometry of the 4f-line and the nature of the interaction lead to different limitations than LC-SLM 4f-line. A detailed review of them can be found in ref [76], and is summarized here.

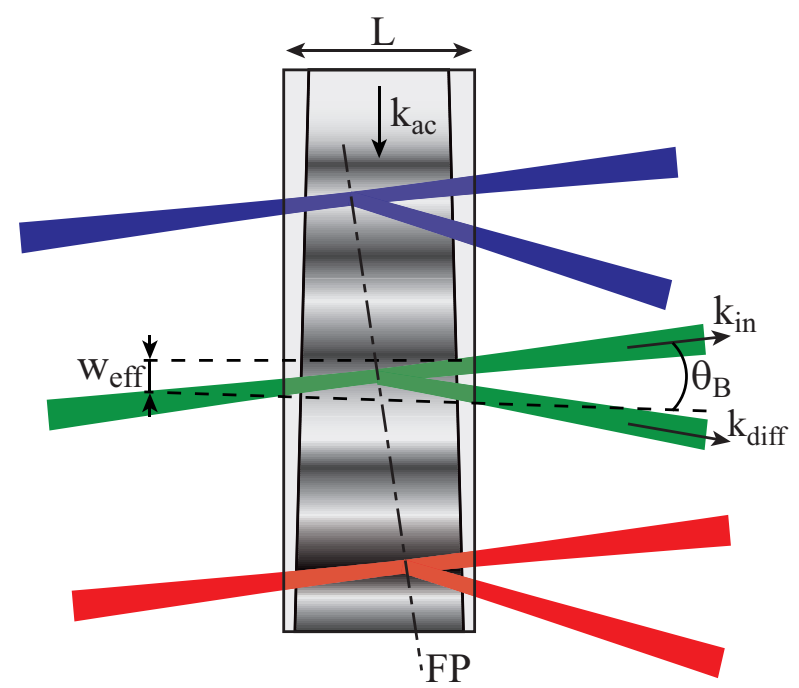

Figure 17. Illustration of the limitations due to the geometry and the thickness of the interaction. The spectrum is dispersed along the vertical direction and three monochromatic frequency components are plotted. To keep the same spatial resolution across the thickness $\mathrm{L}$ of the AOM, the average diameter of the beam $\mathrm{w}_{\text {eff }}$ must be less than the minimum acoustic feature. Due to the phase matching angle $\left(\theta_{B}\right)$, the Fourier plane (FP, dashed dot line) is not in the median plane of the crystal. This limits the available aperture of the device.

- Each acoustic grating pattern appears frozen during the interaction with a femtosecond pulse. As it has to be synchronized with the optical pulse, the 
repetition rate of the laser is limited. An optical pulse is diffracted each physical refreshing time $\Delta \tau$ of the modulator. With the numerical values above[76], this gives a repetition rate that has to be below $100 \mathrm{kHz}$.

- Due to the phase matching angle $\theta_{B}$, the effective optical spot size has a diameter $\mathrm{w}_{\text {eff }}$ which should be smaller than the minimum acoustic feature in order to maximize the resolution. Moreover, also due to this phase matching angle, the Fourier plane (FP, dashed dot line) is not in the median plane of the crystal. To allow interactions with a good resolution, the focus of each spectral component should be included in the crystal (modulo their Rayleigh length $z_{R}$ ). Thus, the angle between the Fourier plane and this median axis fixes the available optical bandwidth and the acoustic frequency (equation 28). As the acoustic bandwidth is generally proportional to the acoustic frequency, this fixes also the minimum acoustic feature and thus the achievable complexity.

- The geometry of the line introduces also dispersive effects. First, the path lengths vary significantly for different optical frequencies (longer for the blue than the red, see figure 12). This effect which is in the first order quadratic has to be compensated for. A second effect is related to the Bragg angle and its dependence on optical frequencies. The mismatch is much more important for short(broadband) pulses and limits the extension of AOM pulse shaping to very short pulses.

- Finally, the absorption of the acoustic wave limits the crystal length and non-linear effects on the acoustic waves imposes to work at relatively low efficiency. Moreover it is sometimes required to work in a closed loop regime in order to compensate for this effect and to reach the desired optical waveform.

\subsubsection{Other masks}

In parallel with the two main spatial light modulators (LC-SLM, AOM), others masks have been developed for specific applications :

- MMDM : This micro-machined deformable mirror is commonly used for wave-front correction of non-linear effects. It is composed of a coated membrane suspended over an array of actuator electrodes on a printed circuit board[90]. The deformation of the reflecting surface is governed by electrostatic force. Such MMDM mask placed in the Fourier plane of a folded $4 \mathrm{f}$-line allows smoothly varying phase only modulation, in addition to low losses and relatively high actuator density. It represents a fairly low cost solution which could be extended on a very broad spectral range depending on the coating of the mirror. Compression of ultrashort pulses down to $15 \mathrm{fs}$ [90] as well as more complex pulses shaping[91] have been obtained.

- MEMMS : This is a micro-electro-mechanical system placed in the Fourier plane of a folded zero dispersion line. It consists of an array of independently addressable piston-type micro-mirrors, which are fabricated by surface-micro-machining. The device is activated by applying a voltage between the mirror and the address electrode, which causes the mirror to move back[92]. The choice of the coating 
allows high efficiency from the UV to the NIR. Compressions as well as complex pulses obtained by phase modulation have been demonstrated by several groups $[92$, 93, 94]. The main drawback is the appearance of strong replicas due to both pixellisation and the large gaps between the mirrors.

- 2D-LC-SLM : This is a phase only LC-SLM consisting of a matrix of pixels. The spectral components of the pulse are spread across the horizontal dimension, and a sawtooth phase function is applied along the vertical direction by the 2D LCSLM to each frequency component[95] (the period of this function determines the direction of the first-order diffracted light). The spatial phase (i.e. vertical position) and amplitude of the sawtooth pattern can be varied for each spectral component to modulate the phase and the amplitude of the diffracted light [57]. Depending on the way the sawtooth phase function is used, a high efficiency or a superior dynamic range of modulation can be obtained [96]. Complex pulse shapes as well as simple compression have been successfully demonstrated[57, 96].

Table 2 gives an outline of the main technical characteristics of available masks presented in this section. Technical details as control type, parameters number, efficiency, refreshing rate and damage threshold, are given when they are available in the cited reference.

\subsection{Shaping by phase transfer}

The second approach to shape the amplitude and the phase of an ultrashort pulse is to perform frequency mixing between the input pulse and a control field $f(t)$ which can be either in the optical or in the acoustic domain.

\subsubsection{Acousto-Optic programmable Dispersive Filters (AOPDF)}

If pulse shaper is one of the oldest and most widespread apparatus for pulse shaping. However an alternative system has appeared in the 90's called AOPDF or Dazzler ${ }^{\mathrm{TM}}$ in its commercial name (Fastlite). It is based on the acousto-optic interaction between a controlled acoustic wave $f(t)$ and an optical ultrashort pulses $E(t)[99,100]$. The interaction is similar to the Bragg diffraction which occurs in chirped mirrors. In this latter, the grating is permanent, fixed by the dielectric coatings. In the AOPDF, the acoustic wave creates a longitudinal transient grating which maximizes the interaction length. Practically, the acousto-optic crystal is highly birefringent for the acoustic as well as for the optical waves. In the crystal, the acoustic wave shapes by diffracting the ordinary incoming optical wave to the diffracted extraordinary optical wave. Thus optical pulse shaping in phase and amplitude is achieved through anisotropic interaction. The change of polarization is produced by the anisotropic interaction which is different from the isotropic interaction in AOM spatial light modulator.

As shown on figure 18, the input optical pulse is chosen to propagate along the ordinary axis(fast axis). 


\begin{tabular}{|c|c|c|c|c|c|c|c|}
\hline Mask & Ref & $\lambda_{0}(\mathrm{~nm})$ & $\begin{array}{l}\text { Control } \\
\text { type }\end{array}$ & $\begin{array}{l}\text { Param. } \\
\text { number }\end{array}$ & Efficiency & $\begin{array}{c}\text { Refresh. } \\
\text { rate } \\
(\mathrm{Hz})\end{array}$ & $\begin{array}{c}\text { Damage } \\
\text { threshold } \\
\left(G W / \mathrm{cm}^{2}\right) \\
\end{array}$ \\
\hline \multirow{3}{*}{$\begin{array}{c}\text { LC } \\
\text { (pixelated) }\end{array}$} & {$[97]$} & 400 & \multirow{3}{*}{$\begin{array}{c}\text { Amplitude } \\
\text { Phase } \\
\text { Polarization }\end{array}$} & $2 * 648$ & $85 \%$ & \multirow{3}{*}{$\sim 10$} & $>1.3(395 \mathrm{~nm}, 30 \mathrm{fs})$ \\
\hline & {$[69]$} & Vis & & $2^{*} 128$ & $85 \%$ & & $4(890 \mathrm{~nm}, 50 \mathrm{fs})$ \\
\hline & {$[60]$} & 800 & & $2 * 640$ & $95 \%$ & & $300(800 \mathrm{~nm}, 45 \mathrm{fs})$ \\
\hline \multirow{5}{*}{$\mathrm{AOM}$} & {$[\overline{[89]}$} & 260 & \multirow{5}{*}{$\begin{array}{c}\text { Amplitude } \\
\text { Phase }\end{array}$} & 170 & $78 \%$ & \multirow{5}{*}{$\sim 10^{5}$} & \multirow[b]{2}{*}{ Fused Silica : NA } \\
\hline & {$[78]$} & 400 & & 200 & NA & & \\
\hline & {$[98]$} & Vis & & 900 & NA & & \multirow{2}{*}{$\mathrm{TeO}_{2}: 0.1$} \\
\hline & {$[76]$} & 800 & & 900 & $30-90 \%$ & & \\
\hline & {$[61]$} & 4900 & & 500 & $80 \%$ & & Germanium:NA \\
\hline \multirow{2}{*}{$\begin{array}{c}\text { MEMM } \\
\text { (pixelated) }\end{array}$} & {$[93]$} & 266 & \multirow[b]{2}{*}{ Phase } & $240 * 200$ & $\mathrm{NA}$ & \multirow[b]{2}{*}{$\sim 10^{2}$} & \multirow{2}{*}{$\begin{array}{c}\text { Aluminium } \\
\text { coating }\end{array}$} \\
\hline & {$[92]$} & 400 & & $240 * 200$ & $85 \%$ & & \\
\hline MMDM & {$[90]$} & 800 & Phase & $3 * 13$ & $97 \%$ & $\sim \sim 10^{3}$ & Gold coating \\
\hline \multirow{2}{*}{$\begin{array}{c}2 \mathrm{D} \mathrm{LC} \\
\text { (pixelated) }\end{array}$} & {$[95]$} & 805 & \multirow{2}{*}{$\begin{array}{c}\text { Amplitude } \\
\text { Phase }\end{array}$} & $480 * 480$ & $\sim 50 \%$ & NA & NA \\
\hline & {$[96]$} & 800 & & $1920 * 1080$ & $\sim 55 \%$ & 60 & NA \\
\hline
\end{tabular}

Table 2. Summary of available Spatial Light Modulators for 4f-line based pulse shapers and few characteristics used in experiments of cited references. LC : liquid crystal, AOM : Acousto-Optic Modulator, MEMM : Micro-Electro Mechanical Mirrors, MMDM : Micro-Machined Deformable Mirror and 2D LC : a 2D Liquid Crystal device. $\lambda_{0}$ is the laser source wavelength used in the references, the control type shows for which shaping SLM can be used, parameter number is the independent points of control of the mask. The efficiency is the transmission (LC), diffraction (AOM) or reflection coefficient. The refreshing rate gives the refreshing pattern frequency of the mask and finally the damage threshold is indicated with the source used for its evaluation. NA : characteristic not available.

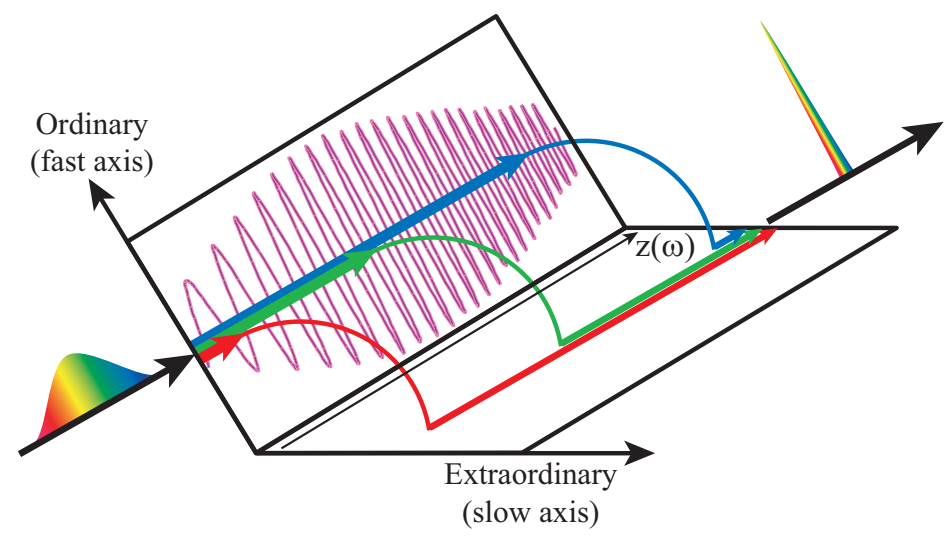

Figure 18. AOPDF principle: By diffraction on the acoustic grating through an acousto-optic interaction, the different spectral components can be switched to the extraordinary axis at different positions in the crystal. 
The different spectral components are diffracted on the extraordinary axis when the wave vectors and frequencies fulfill the phase matching condition and energy conservation:

$$
\begin{aligned}
& \vec{k}_{d i f f}\left(\omega_{d i f f}\right)=\vec{k}_{a c}\left(\omega_{a c}\right)+\vec{k}_{i n}\left(\omega_{i n}\right) \\
& \omega_{d i f f}=\omega_{i n}+\omega_{a c} \simeq \omega_{i n}
\end{aligned}
$$

where diff denotes the diffracted optical beam, in the input optical beam and $a c$ the acoustic wave. This means that the spectral components can be switched on the extraordinary axis at different positions in the crystal, by changing the frequency $\omega_{a c}$ along the crystal. As the group velocities on the ordinary axis and the extraordinary axis are different, one gets a different arrival time at the output of the crystal for each wavelengths. This time depends on the phase matching between the acoustic and the optical frequencies. Moreover the acoustic intensity at the switch location controls the amplitude of the diffracted spectral component.

The shape of the diffracted wave thus depends on both the switch position and the local acoustic intensity. One can write in the spectral domain :

$$
\tilde{E}_{\text {diff }}(\omega) \propto \tilde{E}_{i n}(\omega) \cdot f(\gamma \omega)
$$

or equivalently in the time domain :

$$
E_{\text {diff }}(t) \propto E_{\text {in }}(t) \otimes f(t / \gamma)
$$

where the scaling factor $\gamma=\omega_{a c} / \omega \simeq\left(v_{a c} \delta n\right) / c\left(\sim 10^{-7}\right.$ in $\left.\mathrm{TeO}_{2}\right) . \delta n$ is the crystal optical anisotropy, $v_{a c}$ the acoustic wave velocity. This factor $\gamma$ allows transfering the acoustic waveform to the shape of the optical wave.

The shaping properties of the AOPDF have different origins than $4 \mathrm{f}$-line shapers and have to be properly defined:

- As for the other pulse shapers, it is possible to define a time window in which it is possible to shape the pulse. This window has a different physical origin than for a 4f-line and is mainly determined by the crystal thickness and its anisotropy. The shortest programmable delay is fixed by a complete propagation along the ordinary axis while the longest delay corresponds to a propagation along the extraordinary axis. The difference between both gives the maximum time window :

$$
T_{\text {max }}=\delta n_{g} \cos ^{2}\left(\theta_{i n}\right) \frac{L}{c}
$$

where $\mathrm{L}$ is the length of the crystal and $\delta n_{g}$ the group index difference. For a 25 $\mathrm{mm} \mathrm{TeO}_{2}$ crystal [100] this gives $T_{\max } \simeq 3 \mathrm{ps}$ at $800 \mathrm{~nm}$ and for a $72 \mathrm{~mm} \mathrm{KDP}$ crystal $T_{\text {max }} \simeq 7 \mathrm{ps}$ at $300 \mathrm{~nm}[101]$. Usually a part of this window is used to selfcompensate for the dispersion due to the propagation in the acousto-optic crystal itself. This leads, in general, to an available time window of few ps . 
- The resolution is given [102] by:

$$
\delta \lambda=\frac{0.8}{\delta n \cos ^{2}\left(\theta_{\text {in }}\right)} \frac{\lambda^{2}}{L}
$$

where $\theta_{\text {in }}$ is the angle between the incident wave vector and a reference crystallographic axis. The resolution is typically equal to $0.25 \mathrm{~nm}$ at $800 \mathrm{~nm}$ and down to $0.1 \mathrm{~nm}$ at $266 \mathrm{~nm}$ for commercially available devices.

- The number of control parameter, $N_{c p}$, is given by the number of resolution points in the programmed diffracted bandwidth. In the case of phase only shaping, this bandwidth has to be broad enough to diffract all the optical components with a nearly constant amplitude. Experimentally, it is set to three times the optical bandwidth, which gives:

$$
N_{c p}=\frac{\Delta \lambda}{\delta \lambda}=\frac{\delta n L}{0.8} \cos ^{2} \theta_{i n} \frac{3 \Delta \lambda}{\lambda^{2}}
$$

For a $72 \mathrm{~mm} \mathrm{KDP}$ crystal $\left(\theta_{i n}=48.5^{\circ}, \delta n=0.045\right.$ and $\Delta \lambda=3 \mathrm{~nm}$ at $\left.410 \mathrm{~nm}\right)$ [101], this gives $N_{c p} \simeq 100$ and around 400 in the IR [102].

- The efficiency depends on the phase matching, the length of the crystal, the merit factor of the crystal, the wavelength and finally the acoustic power. A detailed theoretical description can be found in [102].

\section{Design and alignment}

Taking into account the value of the scaling factor $\gamma$, the acoustic wave should have a frequency value around tens of $\mathrm{MHz}$ to allow the shaping of visible wavelength (hundreds of $\mathrm{THz}$ ). The acoustic wave is a progressive wave which is synchronized with the optical wave. It is generated by a transducer glued on the crystal and monitored by a high frequency synthesizer (cf figure 19).

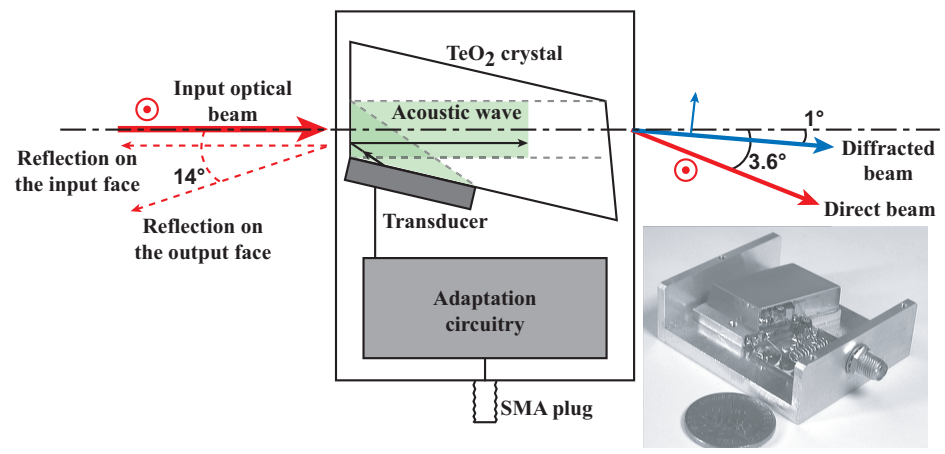

Figure 19. Experimental view of the AOPDF (courtesy of N. Forget) : the optical wave propagates collinearly with the acoustic waves induced by the transducer glued on the acousto-optic crystal (here $\mathrm{TeO}_{2}$ ). The optical wave is diffracted on the acoustic wave and the shaped pulse goes out of the AOPDF following the diffracted beam direction. The size of the apparatus is few $\mathrm{cm}$ in the IR-visible and around $10 \mathrm{~cm}$ in the UV.

The crystal angle is chosen to maximize the compromise between efficiency and resolution [102]. 
In practice, two experimental parameters are important :

- The alignment of the beam within the AOPDF has to be performed carefully. The beam has to enter the AOPDF horizontally, in the center and normally to the inner face (auto-collimation). The best resolution is achieved when the beam is carefully collimated. The fine angular alignment of the device is made by shaping the diffracted optical beam with a thin hole in amplitude and looking at its spectrum. The corresponding spectrogram will present a hole which can be spectrally shifted by rotating the AOPDF. Once the hole is at the same wavelength than the programmed one, the phase matching condition is fulfilled and the AOPDF aligned.

- Due to the difference of speed in the crystal, the acoustic wave is seen frozen by the optical one. However, it is a traveling wave and it has to be precisely synchronized with the optical wave. To avoid any spectral clipping, the acoustic wave should be centered in the crystal when the optical waves enters in it.

Results

The AOPDF has been often used at the input of an amplifier in order to compensate for high order dispersion leading to impressive results in the IR [103]. Intense shaped pulses have also been obtained directly at the output of a visible amplifier [104], leading to pulse duration around $30 \mathrm{fs}$ (see figure 20 (a) : output autocorrelation, (b) output spectrum). Many other applications have been demonstrated as high harmonic tunability [105], compression of fibre amplifiers output [106], coherent control experiments [107]. AOPDF are now available for wavelength ranging from $200 \mathrm{~nm}$ $[108,101]$ to $2 \mu \mathrm{m}$. The figure 20 (c) and (d) present a 13-pulse sequence obtained at $266 \mathrm{~nm}$. Improvements are under studies to fabricate an AOPDF in the Mid-IR [109].

\section{Limitations}

- Main limitations of the AOPDF are related to the fact that it is basically an acoustooptic modulator. For example, the traveling time of the acoustic wave in the crystal limits the repetition rate of the laser : the acoustic wave has to be refreshed for each incoming optical pulse, which limits the repetition rate to hundreds of $\mathrm{kHz}$. As in AOM 4f-line, non-linear effects as well as absorption will also occur on the acoustic waves.

- Other limitations are strongly linked to the nature of the interaction : Shaping is done by delaying the different spectral components. So on the one hand, in order to have a good efficiency in the interaction, the acoustic wave has to be spread along the whole crystal and on the other hand, the spreading of the acoustic wave in the crystal is mainly governed by the programmed shape. This means for example that programming a strongly chirped pulse will lead to a more efficient interaction than 

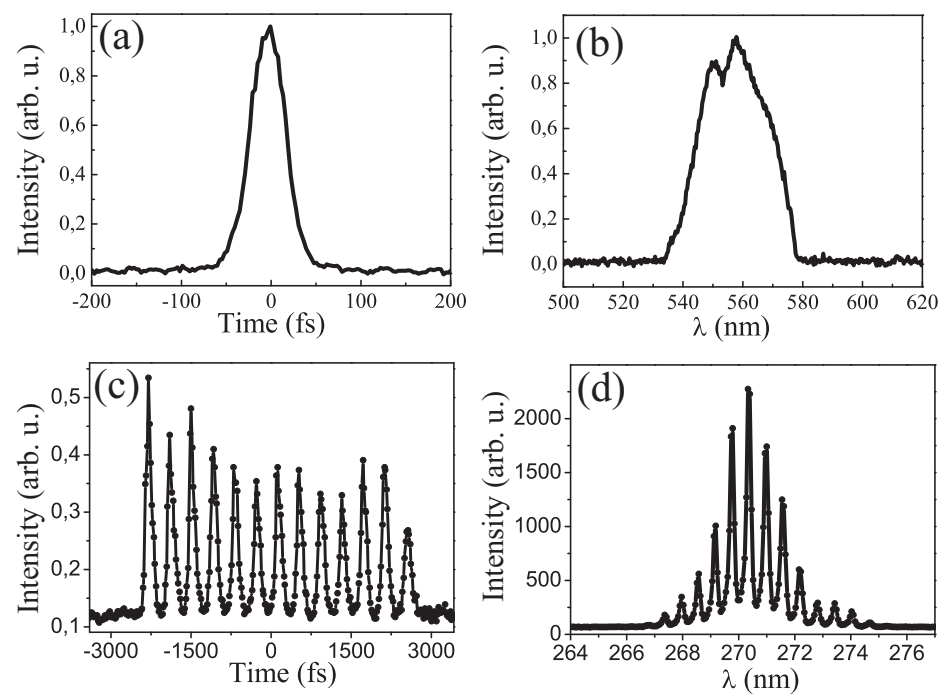

Figure 20. Results obtained with an AOPDF. In the visible range: (a) autocorrelation of the output of a NOPA compressed by the AOPDF and (b) corresponding spectrum. In the UV range $(270 \mathrm{~nm})$ : cross-correlation of a 13-pulse sequence with a reference ultrashort pulse at $800 \mathrm{~nm}$ (c) and corresponding spectrum (d).

programming a short pulse which implies that all the optical frequencies will be diffracted on a very short time [110].

- Non-linear effects on the optical waves will be more important due to the fact that the pulse is not spatially dispersed as it is in a $4 \mathrm{f}$-line, leading to higher fluence.

- Finally, as frequency components are diffracted at different position in the crystal, it will suffer from spatio-temporal coupling.

\subsubsection{Non-linear frequency mixing}

The prospect to generate arbitrarily shaped optical pulse has triggered significant developments in many fields of fundamental and applied research. This leads to a great effort to extend the spectral ranges where such shaped pulses are available. One way which has been detailed above consists on developing pulse shapers in different spectral range. Another option consists on using non-linear mixing to transfer the shape of the control pulse obtained easily in the NIR range to another wavelength range. Interesting results have been obtained in the mid-IR [111, 112] as well as in the UV range [113, 114]. However, this phase transfer occurs with constraints in terms of phase matching conditions, energies, bandwidth. For example, when sum frequency mixing a control field with an independent field, the technique is linear. However, this is no longer true when generating the second harmonic of a control field. In particular many works have demonstrated that the shape of the input pulse can completely alter the bandwidth of the SHG-generated pulse $[115,4]$. As demonstrated in $[116,117]$, the use of two chirped pulses allows a very efficient generation of second harmonic picosecond pulses. 


\subsection{Conclusion on pulse shaping}

This section, albeit many pages long, only shows the tip of the iceberg of pulse shaping. Before listing some achievements using different kind of pulse shapers, we would like to remind few facts to keep in mind when dealing with a pulse shaper:

- The shortest feature at the output of the pulse shaper will always be governed by the available optical bandwidth.

- The maximum temporal window $T$ will be given by the set-up as well as the optical beam properties (see section 3.2.2).

- Arbitrary pulse shapers provide a complexity $\eta$ of few hundreds. This gives the ratio between the bandwidth and the minimum spectral feature available in the spectral domain or $T / \delta t$ in the time domain. Sometimes a simpler device allows achieving higher complexity (see Table 1).

Finally more than hundreds of references in various fields are using pulse shapers. The table 3 presents some realizations of pulses shapers. They are sorted by shaper type and wavelength range. When available, the input pulse properties are given as well as the shaping type. Then main results are specified. This table doesn't mention all the specific references which detail analysis, limitations of those techniques. For this information, the reader should refer to the corresponding sections above.

\begin{tabular}{|c|c|c|c|c|c|}
\hline \multirow{2}{*}{$\begin{array}{l}\text { Shaper } \\
\text { type }\end{array}$} & \multirow[t]{2}{*}{ Ref } & \multicolumn{2}{|c|}{ Source parameter } & \multirow{2}{*}{$\begin{array}{l}\text { Shaping } \\
\text { type }\end{array}$} & \multirow[t]{2}{*}{ Main results } \\
\hline & & $\lambda_{0}(\mathrm{~nm})$ & $\Delta t(\mathrm{fs})$ & & \\
\hline \multirow[b]{5}{*}{ 4f-line } & \multirow[t]{2}{*}[97]{} & 400 & 35 & \multirow[t]{2}{*}{$\mathrm{P}$} & \multirow{2}{*}{$\begin{array}{l}\text { Compression of ultrashort pulse } \\
\text { in UV and IR using LC mask }\end{array}$} \\
\hline & & 800 & 40 & & \\
\hline & {$[69]$} & Vis & $<20$ & $\mathrm{P}-\mathrm{A}$ & $\begin{array}{l}\text { Closed-loop compression down to } 16 \text { fs } \\
\text { from a Non collinear Optic Amplifier } \\
\text { (NOPA) }\end{array}$ \\
\hline & [118] & Vis & NA & $\mathrm{P}-\mathrm{A}$ & $\begin{array}{l}\text { White-light shaping and amplification. } \\
\text { Production of different color pulses }\end{array}$ \\
\hline & {$[67]$} & 778 & 30 & Pol & $\begin{array}{l}\text { Control of two photon absorption using } \\
\text { polarization shaping }\end{array}$ \\
\hline \multirow{5}{*}{ (pixelated) } & {$[60]$} & 795 & 100 & $\mathrm{P}-\mathrm{A}$ & $\begin{array}{l}\text { High resolution }(0.06 \mathrm{~nm} / \text { pixels }) \text { phase } \\
\text { and amplitude pulse shaper }\end{array}$ \\
\hline & [119] & 800 & 70 & $\mathrm{P}-\mathrm{A}$ & $\begin{array}{l}\text { First use of two } 4 \text {-line shaper for } \\
\text { amplitude and phase control }\end{array}$ \\
\hline & {$[68]$} & 800 & 13 & $\mathrm{P}$ & $\begin{array}{l}\text { Complex phase shaping of ultra-broad- } \\
\text { bandwidth pulses }\end{array}$ \\
\hline & {$[56]$} & 800 & 80 & Pol & Polarization pulse shaping \\
\hline & {$[70]$} & 800 & 20 & $\mathrm{P}$ & $\begin{array}{l}\text { Phase shaping improvement using } 512 \\
\text { pixels }\end{array}$ \\
\hline
\end{tabular}




\begin{tabular}{|c|c|c|c|c|c|}
\hline \multirow{2}{*}{$\begin{array}{l}\text { Shaper } \\
\text { type }\end{array}$} & \multirow[t]{2}{*}{ Ref } & \multicolumn{2}{|c|}{ Source parameter } & \multirow{2}{*}{$\begin{array}{l}\text { Shaping } \\
\text { type }\end{array}$} & \multirow[t]{2}{*}{ Main results } \\
\hline & & $\lambda_{0}(\mathrm{~nm})$ & $\Delta t(\mathrm{fs})$ & & \\
\hline & {$[120]$} & 800 & 30 & $\mathrm{P}$ & $\begin{array}{l}\text { Robust and flexible set-up for pulse } \\
\text { shaping }\end{array}$ \\
\hline & {$[121]$} & 804 & 50 & $\mathrm{P}$ & $\begin{array}{l}\text { High resolution }(0.15 \mathrm{~nm} / \text { pixel }) \text { phase } \\
\text { shaping using } 640 \text { pixels }\end{array}$ \\
\hline & {$[122]$} & 800 & 4.3 & $\mathrm{P}$ & $\begin{array}{l}\text { Pulse shaping of octave spanning fs pulses } \\
\text { with prisms-pulse shaper. Source : } \\
\text { oscillator }\end{array}$ \\
\hline & {$[123]$} & 800 & 6 & $\mathrm{P}$ & $\begin{array}{l}\text { Pulse shaping of octave spanning fs pulses } \\
\text { with prisms-pulse shaper. Source : } \\
\text { Amplifier+argon filled glass capillary fibre }\end{array}$ \\
\hline \multirow{5}{*}{ 4f-line } & {$[89]$} & 260 & 55 & \multirow{9}{*}{$\mathrm{P}-\mathrm{A}$} & $\begin{array}{l}\text { Deep UV pulse shaping using fused silica } \\
\text { AOM }\end{array}$ \\
\hline & {$[78]$} & 400 & 100 & & $\begin{array}{l}\text { First implementation of direct UV shaping } \\
\text { using AOM }\end{array}$ \\
\hline & {$[80]$} & 400 & 100 & & $\begin{array}{l}\text { Closed-loop pulse compression in the UV } \\
\text { and optimization of CARS signal }\end{array}$ \\
\hline & [98] & Vis & NA & & $\begin{array}{l}\text { Amplification and shaping of white light } \\
\text { from a NOPA. } 70 \mathrm{~nm} @ 620 \mathrm{~nm} \text {. }\end{array}$ \\
\hline & {$[75]$} & 620 & 180 & & $\begin{array}{l}\text { First implementation of AOM based pulse } \\
\text { shaping }\end{array}$ \\
\hline \multirow[t]{4}{*}{$\mathrm{AOM}$} & {$[81]$} & 777 & 30 & & $\begin{array}{l}\text { Closed-loop optimization of the multipho- } \\
\text { ton transfer in the strong field regime }\end{array}$ \\
\hline & {$[76]$} & 780 & 90 & & $\begin{array}{l}\text { Theoretical and experimental study of the } \\
\text { limitations in AOM based pulse shaping }\end{array}$ \\
\hline & {$[79]$} & 795 & 150 & & $\begin{array}{l}\text { Amplified pulse shaping (pulse shaper } \\
\text { placed in front of the amplifier })(200 \mu J) \text {. }\end{array}$ \\
\hline & {$[61]$} & 4900 & 140 & & $\begin{array}{l}\text { Direct pulse shaping in the mid IR. } \\
\text { Efficiency up to } 30 \%\end{array}$ \\
\hline \multirow[b]{4}{*}{$\mathrm{AOPDF}$} & {$[108]$} & $\begin{array}{l}270 \\
330 \\
404\end{array}$ & 55 & & $\begin{array}{l}\text { Complex and tunable shaping in the UV } \\
\text { using AOPDF with KDP crystal }\end{array}$ \\
\hline & {$[101]$} & 402 & NA & & $\begin{array}{l}\text { Theory and experiments on UV AOPDF. } \\
\text { Efficiency up to } 50 \% \text {. }\end{array}$ \\
\hline & {$[104]$} & $\begin{array}{l}500- \\
650\end{array}$ & 30 & & $\begin{array}{l}\text { Shaping at the output of a NOPA. } \\
\text { Efficiency up to } 50 \% \text {. }\end{array}$ \\
\hline & {$[107]$} & $\sim 570$ & $\begin{array}{c}\Delta \lambda= \\
60 \mathrm{~nm}\end{array}$ & $\mathrm{P}-\mathrm{A}$ & $\begin{array}{l}\text { Optimal Control and enhancement of the } \\
\text { three-photon fluorescence from molecular } \\
\text { iodine }\end{array}$ \\
\hline
\end{tabular}




\begin{tabular}{|c|c|c|c|c|c|}
\hline \multirow{2}{*}{$\begin{array}{l}\text { Shaper } \\
\text { type }\end{array}$} & \multirow[t]{2}{*}{ Ref } & \multicolumn{2}{|c|}{ Source parameter } & \multirow{2}{*}{$\begin{array}{l}\text { Shaping } \\
\text { type }\end{array}$} & \multirow[t]{2}{*}{ Main results } \\
\hline & & $\lambda_{0}(\mathrm{~nm})$ & $\Delta t(\mathrm{fs})$ & & \\
\hline & {$[100]$} & 800 & 17 & & $\begin{array}{l}\text { First shaping using } \mathrm{TeO}_{2} \text { AOPDF. Com- } \\
\text { pression down to } 17 \text { fs was achieved by fine } \\
\text { phase and gain narrowing correction }\end{array}$ \\
\hline & {$[103]$} & 800 & 10 & & $\begin{array}{l}\text { Production of terawatt-scale sub-10-fs } \\
\text { laser pulse at } 1 \mathrm{kHz} \text { repetition rate }\end{array}$ \\
\hline & {$[105]$} & 810 & 30 & & $\begin{array}{l}\text { Enhancement of the efficiency and tuning } \\
\text { of the High Harmonic Generation using a } \\
\text { closed-loop }\end{array}$ \\
\hline \multirow{6}{*}{$\begin{array}{l}\text { Phase } \\
\text { transfer }\end{array}$} & {$[113]$} & 200 & 155 & $\mathrm{~A}$ & $\begin{array}{l}\text { Amplitude shaping at } 200 \mathrm{~nm} \text { using SFM } \\
\text { of a shaped fundamental pulse and THG } \\
\text { pulse }\end{array}$ \\
\hline & {$[114]$} & 344 & 19 & $\mathrm{P}-\mathrm{A}$ & $\begin{array}{l}\text { UV shaping using SFM between a shaped } \\
\text { NOPA pulse and a stretched IR pulse }\end{array}$ \\
\hline & {$[124]$} & 400 & NA & POL & $\begin{array}{l}\text { UV polarization shaping by SHG in } \\
\text { two non-linear crystals of a polarization- } \\
\text { shaped IR pulses. }\end{array}$ \\
\hline & {$[125]$} & $3000-10000$ & $\sim 200 \mathrm{fs}$ & $\mathrm{P}$ & $\begin{array}{l}\text { Mid IR Phase shaping by DFM of the } \\
\text { signal }(1.1-1.6 \mu \mathrm{m}) \text { of an OPA and the idler }\end{array}$ \\
\hline & {$[126]$} & 3300 & NA & $\mathrm{P}-\mathrm{A}$ & $\begin{array}{l}\text { Mid IR Phase shaping by OPA process } \\
\text { using shaped IR pump pulse by an AOM } \\
\text { placed in a } 4 \mathrm{f} \text { line }\end{array}$ \\
\hline & {$[111]$} & 12000 & NA & $\mathrm{P}$ & $\begin{array}{l}\text { Phase shaping in the IR by DFM between } \\
\text { two fundamental chirped pulses }\end{array}$ \\
\hline 4f-line+ & {$[93]$} & 266 & 100 & \multirow{3}{*}{$\mathrm{P}$} & $\begin{array}{l}\text { Compression of deep UV broadened pulses } \\
\text { using a closed-loop }\end{array}$ \\
\hline \multirow{2}{*}{$\begin{array}{l}\text { MEMM } \\
\text { (pixelated) }\end{array}$} & {$[94]$} & 320 & 30 & & $\begin{array}{l}\text { Phase control of tunable UV pulse and two } \\
\text { photon autocorrelation }\end{array}$ \\
\hline & {$[92]$} & 404 & 80 & & $\begin{array}{l}\text { First shaping using MEMM. Compression } \\
\text { and sinusoidal modulation }\end{array}$ \\
\hline \multirow{2}{*}{$\begin{array}{l}\text { 4f-line+ } \\
\text { MMDM }\end{array}$} & {$[90]$} & 800 & 15 & $\mathrm{P}$ & $\begin{array}{l}\text { High order compression down to } 15.2 \text { fs } \\
\text { using a closed-loop approach }\end{array}$ \\
\hline & {$[91]$} & 842 & $\sim 100$ & $\mathrm{P}$ & $\begin{array}{l}\text { Precise phase control and characterization } \\
\text { using negative-feedback }\end{array}$ \\
\hline \multirow{2}{*}{$\begin{array}{c}\text { 2D LC } \\
\text { (pixelated) }\end{array}$} & {$[95]$} & 805 & 40 & $\mathrm{P}-\mathrm{A}$ & $\begin{array}{l}\text { Amplitude and phase shaping using a } \\
\text { phase only } 2 \mathrm{D} \text { spatial light modulator }\end{array}$ \\
\hline & {$[96]$} & 800 & $\sim 50$ & $\mathrm{P}-\mathrm{A}$ & $\begin{array}{l}\text { Comparison between two pulse shaping } \\
\text { scheme in term of efficiency and modula- } \\
\text { tion }\end{array}$ \\
\hline
\end{tabular}




\begin{tabular}{|c|c|c|c|c|c|}
\hline $\begin{array}{c}\text { Shaper } \\
\text { type }\end{array}$ & Ref & \multicolumn{2}{|c|}{ Source parameter } & Shaping & Main results \\
\cline { 3 - 3 } & & $\lambda_{0}(\mathrm{~nm})$ & $\Delta t(\mathrm{fs})$ & type & \\
\hline \hline
\end{tabular}

Table 3: Table of arbitrary pulse shaping experimental demonstrations; the first column mentions the shaper type, the second column, the citation number, the third and fourth column are the main source parameters (central wavelength, duration). The shaping type can be P : phase only, P-A : Phase and Amplitude pulse shaping or Pol : polarization pulse shaping. Finally, in the last column, the main results of the reference are presented. NA : Characteristic not available. SFM : Sum Frequency mixing; DFM : Difference Frequency Mixing. SHG : Second Harmonic Generation. THG : Third Harmonic Generation.

\section{Characterization of ultrashort shaped pulses}

\subsection{Prerequisite and generality}

When looking for a characterization technique, beginners can be puzzled by the number of available methods. Indeed, there exists a vast variety of methods, ranging from simplistic and limited to the most complex and refined one. We won't described all of them here and interested readers are invited to read a recent review in this domain [13] and the various articles of a special issue of J. Opt. Soc. Am. B on pulse characterization [127] for a more exhaustive overview. We will restrict ourselves to the most widely used methods, lying in three categories with increasing degree of complexity and refinement. Methods lying in the first category are said to provide incomplete characterization of the pulse. They usually give an estimate of the pulse duration or of the pulse intensity envelope without allowing a full reconstruction of electric field $\mathcal{E}(t)$. The most widely used methods for incomplete characterization are presented in 4.2. The two other categories contain referenced complete characterization methods, and self-referenced complete characterization methods. All these methods allow a full reconstruction of the electric field $\mathcal{E}(t)$. The main difference between the first and the second category is that methods lying in the first one necessitate the use of a fully characterized reference pulse (see 4.3) whereas methods in the latter category do not need any (see 4.4).

In the following, we will indifferently refer to a pulse in the time domain by either its real electric field $\mathcal{E}(t)$ or its complex counterpart $E(t)$ as the latter usually leads to simpler equations. 


\subsection{Incomplete characterization methods}

These methods are among the easiest to implement and are extremely widely used. However, they provide limited insight in the pulse structure but are nonetheless useful for a quick estimate of the pulse duration. The two methods we will present here are based on non-linear correlation in a $\chi^{(2)}$ crystal, namely pulse autocorrelation and crosscorrelation between two different pulses. The former is more adapted to pulses close to the Fourier transform limit whereas the latter can be used to get some insight on the time structure of a shaped pulse using an ancillary short pulse.

In the following, we will assume that the $\chi^{(2)}$ crystal used is thin enough to avoid any spectral windowing due to phase matching.

\subsubsection{Autocorrelation}

Autocorrelation is one of the oldest and most widely used method to estimate the duration of a short pulse. The principle is the following: the test pulse is split in two delayed replicas in a Michelson interferometer, both replicas are focused and spatially overlapped inside a $\chi^{(2)}$ crystal. The intensity of the generated second harmonic is recorded as a function of the delay. When both replicas are overlapping in time inside the crystal, the second harmonic signal is increasing: the better the overlap, the higher the second harmonic signal. There exists two different kind of autocorrelators: background free intensimetric ones [128] and interferometric ones[129].

Intensimetric autocorrelator is described on figure 21: the two delayed replicas are focused in the crystal with an angle. The second harmonic generated inside the crystal contains three contributions: the upconversion of the first replica $E(t)$ with itself, the upconversion of the second replica $E(t-\tau)$ with itself and the cross-term between both replicas. Due to momentum conservation rules, each contribution exits the crystal in a different direction, the cross-term being on axis. Using a spatial filter, this term is selected and sent to a slow photodiode. The electric field impinging on the photodiode

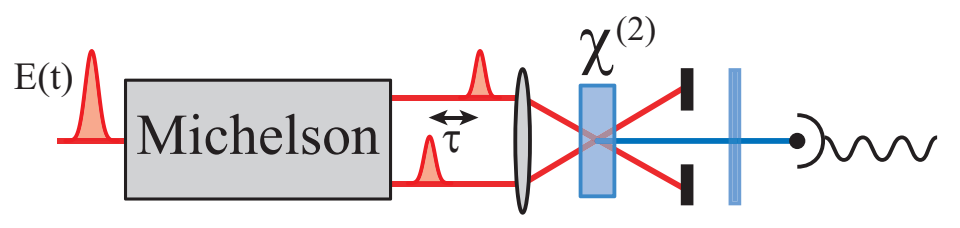

Figure 21. Intensimetric autocorrelator: the two delayed replicas are focused in the crystal with an angle and only the cross-term of the second harmonic is recorded.

can thus be written as $E_{2}(t, \tau) \propto E(t) E(t-\tau)$. The photodiode is assumed to be slow compared to the pulse duration: in other words, the electrical signal it provides is proportional to the integrated intensity of $E_{2}(t, \tau)$ :

$$
S(\tau) \propto \int\left|E_{2}(t, \tau)\right|^{2} d t \propto \int I(t) I(t-\tau) d t
$$


The intensimetric autocorrelator thus gives access to the autocorrelation function of the intensity envelope of the test pulse. It is said to be "background free" as the signal goes back to zero when the two replicas are not overlapping in time. The main advantage of such a device is its simplicity. However, it provides limited information on the pulse itself. Indeed, to estimate a pulse duration from the autocorrelator signal, one has to make an assumption on the pulse profile [128]. Moreover, the autocorrelation function is symmetric in time, which means that a pulse preceded by a pre-pulse will give the same signal than a pulse followed by a post-pulse. This time ambiguity can be particularly limiting when trying to compensate for cubic phase terms. Last, the intensimetric autocorrelator does not provide any information on what's below the envelope. In particular, a chirped pulse can give exactly the same autocorrelation signal than a longer Fourier limited pulse.

The interferometric autocorrelator differs from the intensimetric one in that both replicas are collinearly focused in the crystal. In this case, the electric field impinging on the photodiode contains all the terms of the second harmonic and the signal is now:

$$
S(\tau) \propto \int|E(t)+E(t-\tau)|^{4} d t
$$

There are two main differences with the signal given by the intensimetric autocorrelator. First, even when both replicas are not temporally overlapped, the signal is not zero as it contains the upconversion signal from each replica: the interferometric autocorrelator is not background free. Second, the interferometric signal exhibits fast oscillations corresponding to the beating between the different terms in the second harmonic generation. By Fourier analysis of these oscillations, the pulse spectrum can be retrieved. Interferometric autocorrelator thus provides both an estimate of the duration and the pulse spectrum, the combination of both allowing an estimate of the time bandwidth product of the test pulse.

\subsubsection{Intensimetric Cross-correlation}

The two autocorrelation methods presented above works well for simple pulses close to Fourier limited. However, they provide limited insight for complex pulses like pulse sequences or strongly shaped pulses exiting from a pulse shaper. For this kind of pulses, intensimetric cross-correlation with a short pulse is better suited. As shown on figure 22, the only difference between cross-correlation and autocorrelation setups is that one of the two replicas of the test pulse is replaced by an ancillary short pulse.

Such a cross-correlation set-up provides with a reconstruction of the intensity profile $I(t)$ of the test pulse, convoluted by the intensity profile of the short ancillary pulse. As an example, figure 10 shows the cross-correlation signal obtained when using a $100 \mathrm{fs}$ ancillary pulse to characterize different complex shaped pulse sequences. One feature of intensimetric cross-correlation is that the delay does not need to be optically stable (i.e. with a jitter lower than the optical period). Moreover, ancillary and test pulses can be 


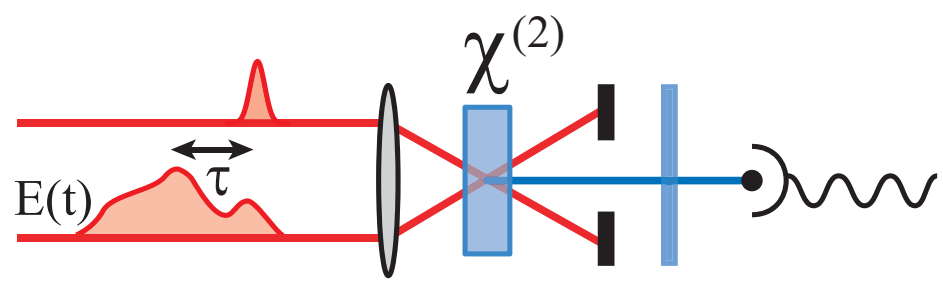

Figure 22. Cross-correlation: The test pulse $E(t)$ is non-collinearly mixed with a short one in a $\chi^{(2)}$ crystal and the sum-frequency signal is recorded as a function of the delay $\tau$ between each pulse.

in different spectral domains. The only constraint is that the ancillary pulse has to be coherent with the test pulse, and short enough to resolve its intensity structure. The main limitation is that intensimetric cross-correlation does not give any information on the phase. As an example, when characterizing a pulse sequence, the cross-correlation signal will be independent on the relative phase between the pulses in the sequence [130].

\subsection{Referenced complete characterization methods}

Methods presented here allow for the full reconstruction of the electric field $\mathcal{E}(t)$ of the test pulse provided a fully characterized reference pulse is available. As we have seen in section 2.1, all the information concerning an ultrashort pulse is contained in its electric field $\mathcal{E}(t)$, or equivalently in both its spectral phase $\varphi(\omega)$ and spectral amplitude $A(\omega)$. A complete characterization of a short pulse should thus give either $\mathcal{E}(t)$ or both $\varphi(\omega)$ and $A(\omega)$. Sadly, for a femtosecond pulse, a direct measurement of $\mathcal{E}(t)$ is not achievable. Indeed, it would require a detector with a time response shorter than the optical cycle of the pulse to characterize (typically few femtosecond in the visible). Such a detector doesn't exist and the fastest detectors exhibit time response around hundreds of femtosecond $\dagger$ Any complete characterization method must thus provide an indirect way to access both $\varphi(\omega)$ and $A(\omega)$.

For that purpose, referenced complete characterization methods make use of a reference pulse which spectral phase $\varphi_{\text {ref }}(\omega)$ and amplitude $A_{r e f}(\omega)$ are perfectly known. The two main referenced techniques are spectral interferometry and Fourier transform spectroscopy. They are conceptually extremely close and completely equivalent, the only difference being that the former lies in the frequency domain whereas the latter is in the temporal domain.

\subsubsection{Spectral interferometry}

Pioneered by Froehly and coworkers [132], spectral interferometry is based on a simple set-up that contains barely more than a spectrometer, as depicted on figure 23 . The test pulse $E(t)$ and the reference pulse $E_{r e f}(t)$ are recombined with a delay $\tau$ and sent

$\dagger$ The only exception to date is the direct measurement of a few-cycle femtosecond pulse by sampling with a XUV attosecond pulse [131]. 
(a)

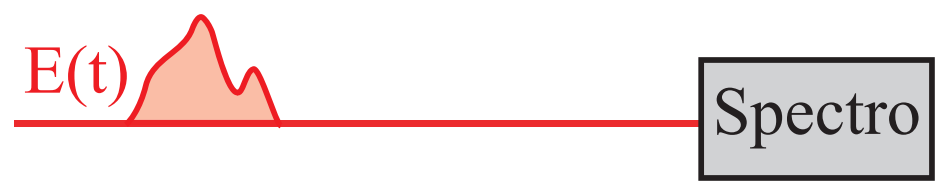

(b)

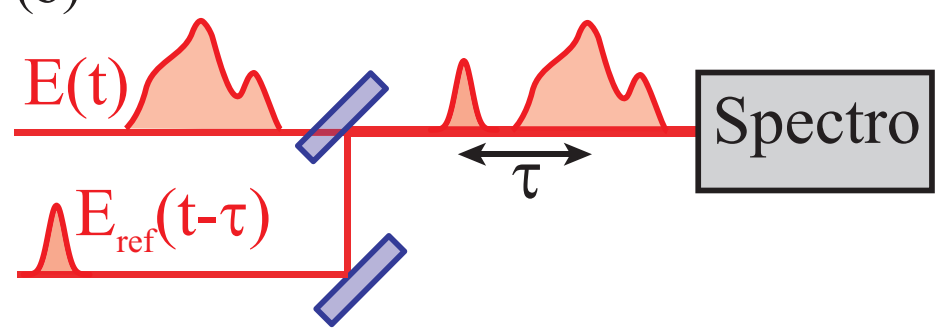

Figure 23. Spectral interferometry: The test pulse $E(t)$ is sent into a spectrometer either (a) alone to measure spectral amplitude or (b) together with a delayed reference pulse $E_{r e f}(t-\tau)$ to retrieve the spectral phase.

into a spectrometer. The complete characterization is a a two step process. First, the reference is blocked and only the test pulse is sent to the spectrometer. As one would expect, the spectrometer gives

$$
I(\omega)=|\tilde{E}(\omega)|^{2}=A^{2}(\omega)
$$

from which the spectral amplitude of the test pulse $A(\omega)$ is directly retrieved. In the second step, both pulses are sent into the spectrometer with a fixed delay $\tau$. The signal from the spectrometer now exhibits fringes that are due to the interference between both pulses:

$$
\begin{aligned}
I(\omega) & =\left|\tilde{E}(\omega)+\tilde{E}_{r e f}(\omega) e^{i \omega \tau}\right|^{2} \\
& =A^{2}(\omega)+A_{r e f}^{2}(\omega)+2 A(\omega) A_{r e f}(\omega) \cos \left[\varphi(\omega)-\varphi_{r e f}(\omega)-\omega \tau\right]
\end{aligned}
$$

The cosine term, responsible for the fringes, contains two contributions. The main one, $-\omega \tau$, is due to the delay and sets the overall fringe spacing. The second one, $\varphi(\omega)-\varphi_{\text {ref }}(\omega)$, codes for the spectral phase difference between the test and reference pulses and locally modify the fringe spacing. In other words, the interferogram can be interpreted in terms of frequency modulation: the delay acts as a carrier frequency modulated by the phase difference. The most common way to extract this phase difference from the interferogram is to use Fourier filtering $[133,134]$ to isolate the oscillating term and extract its argument. We first take the Fourier transform of $I(\omega)$. As depicted on figure 24, it consists of three lobes respectively centered around time $-\tau, 0$, and $\tau$. The latter is the one containing the phase information we care about. We select this side-lobe by applying a window filter around it. Last, we Fourier transform 
(a)

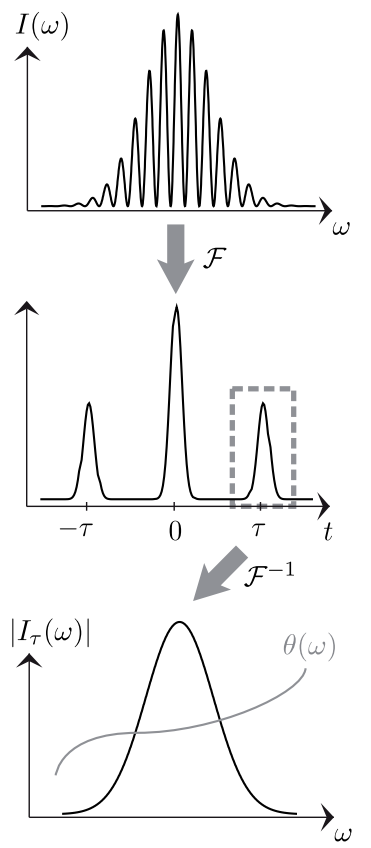

(b)
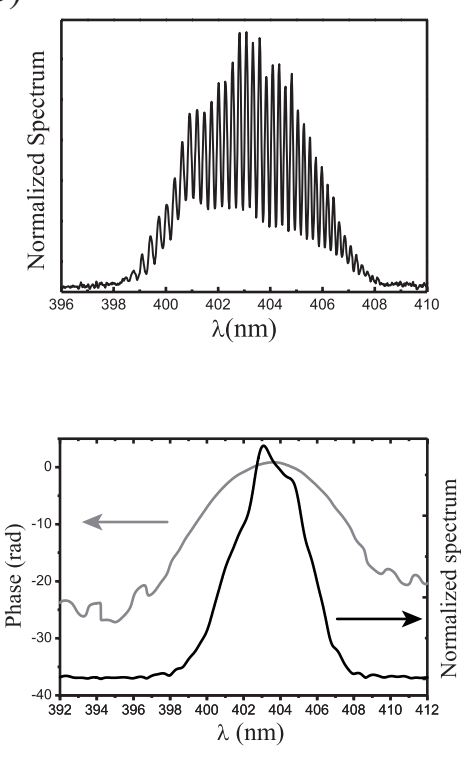

Figure 24. Fourier filtering for spectral interferometry. (a) Principle: the interferogram is Fourier transformed and only one side lobe is kept and back Fourier transformed, giving access to the relative spectral phase $\theta(\omega)$. (b) Top : Experimental interferogram using two UV pulses, one Fourier-limited and one chirped; Bottom : The retrieved spectrum in black, the retrieved relative phase (gray) is quadratic.

back this side-lobe. This gives the following complex function

$$
I_{\tau}(\omega)=A(\omega) A_{r e f}(\omega) \exp \left[i\left(\varphi(\omega)-\varphi_{r e f}(\omega)-\omega \tau\right)\right]
$$

from which we can extract the argument:

$$
\theta(\omega)=\varphi(\omega)-\varphi_{\text {ref }}(\omega)-\omega \tau
$$

As $\varphi_{\text {ref }}(\omega)$ is a known quantity, we can retrieve $\varphi(\omega)$ modulo a linear phase term $-\omega \tau$. This linear term simply corresponds to a temporal delay and does not modify the shape of the test pulse. We usually remove the linear component of $\varphi(\omega)$ to center the retrieved $\mathcal{E}(t)$ at $t=0$.

Spectral interferometry thus provides a measurement of both $A(\omega)$ and $\varphi(\omega)$ from which the electric field $\mathcal{E}(t)$ can be reconstructed. However, several conditions must be fulfilled. First, in order to get interferences, the test and reference pulses need to be mutually coherent (out of the same laser source). Moreover, as can be seen in equation 44, the phase can only be reconstructed where both the spectral amplitude of the test and reference pulses are non-zero. It means that the spectral amplitude of the reference pulse needs to encompass that of the test pulse. The last two conditions concern the delay $\tau$. First, $\tau$ needs to be small enough for the fringes to be correctly sampled on the spectrometer. If $\delta \omega$ is the spectral resolution of the spectrometer, in order to get at least six points per period $\ddagger$, we need to fulfill $\tau<\tau_{\max }=2 \pi /(6 \delta \omega)$, $\ddagger$ In theory, the Shannon criterion says 3 points is enough. In practice, we recommend 6 points 
which sets an upperbound for $\tau$. Second, there is also a lower bound for $\tau$ that originates from the Fourier filtering. Indeed, for it to work correctly, the three lobes at $-\tau, 0, \tau$ should be well separated. This implies that $\tau>3 T$, where $T$ is the duration of longest of both the reference and test pulse.

\subsubsection{Fourier transform spectroscopy}

Fourier transform spectrometry [134] contrary to its spectral counterpart does not necessitate a spectrometer. Instead, it relies on a simple photodiode which signal is recorded as a function of a variable delay $\tau$ between two pulses. It is particularly interesting for spectral regions where spectrometers can be hard to find like in the midIR for example.

As for spectral interferometry, the retrieval of the test pulse is a two step process: the temporal interferences between two replicas of the test pulse give access to the spectral amplitude whereas the temporal interferences between reference and test pulses allow retrieving the spectral phase. As the photodiode is supposed to be slow, it acts as an integrating detector and provides a signal

$$
S_{A}(\tau)=\int|E(t)+E(t-\tau)|^{2} d t
$$

for amplitude measurement and

$$
S_{\varphi}(\tau)=\int\left|E(t)+E_{r e f}(t-\tau)\right|^{2} d t
$$

for phase measurement.

In both cases, the Fourier transform of the interferogram reveals three components at $-\omega_{0}, 0$ and $\omega_{0}$, where $\omega_{0}$ is the centre frequency of the test pulse. The term at $\omega_{0}$ contains all the information we need. For amplitude measurement this term gives $\tilde{E}(\omega) \tilde{E}^{*}(\omega)=A^{2}(\omega)$, which is the spectral intensity of the test pulse, whereas for phase measurement this term now reads $\tilde{E}(\omega) \tilde{E}_{r e f}^{*}(\omega)$, which argument is the relative phase $\theta(\omega)$ between test and reference pulses. From these two quantities and provided the reference is fully characterized, one can reconstruct the test pulse.

However, there are some constraints on the acquisition of both interferograms for this technique to work correctly: in order to avoid aliasing in the Fourier transform, the time step $\delta t$ should be smaller than the optical period $t_{0}=2 \pi / \omega_{0}$. In practice $\delta t \leq t_{0} / 6$ is enough. Once the time step is fixed, the number of acquired points $N$ set the spectral resolution $\delta \omega$ with which both $A(\omega)$ and $\varphi(\omega)$ will be reconstructed. The needed resolution depends on the structure of the spectral phase and amplitude. For a smooth bellshaped spectrum with a slow varying phase, a couple of tens of points across the spectrum is usually enough. Moreover, as for the spectral interferometry, the reference pulse needs to be coherent with the test one and its spectrum has to encompass that of the test pulse. 


\subsection{Self-referenced complete characterization methods}

The methods for referenced complete characterization presented above contains a huge loophole. Indeed, they rely on a fully characterized referenced pulse. Moreover, the reference pulse and the test pulse need to have similar spectral properties (central frequency, bandwidth, ...). In other words, referenced characterization methods can solve the problem of ultrashort pulse characterization provided it has already been solved once... But how do we characterize the first reference pulse?

The solution is to use a self-referenced complete characterization technique that does not require a reference or in other words, that takes the test pulse as a reference for its own measurement. Methods lying in this category are the most powerful for short pulse characterization in the sense that they do not use any prior knowledge to achieve the reconstruction of the test pulse electric field $\mathcal{E}(t)$. However, this comes with a price and these methods put high requirements on the experimental set-up. In particular they require at least non-linear or non-stationary optics. The reason for these requirements will be explained in section 4.4.1. We will then present both of the two widely used selfreferenced complete characterization techniques: FROG in section 4.4.2 and SPIDER in section 4.4.3.

\subsubsection{Prerequisite for self-referenced measurement of $\varphi(\omega)$}

What do we need to completely characterize a test pulse $\mathcal{E}(t)$ without resorting to a reference pulse? Can we use "usual optics", that is time-stationary linear optics? We can always try. As we have seen in section 3.1, any time-stationary linear optical set-up can be described by a complex transfer function $H(\omega)$. If we input a test pulse $\tilde{E}(\omega)$ we get at the output:

$$
\tilde{E}_{\text {out }}(\omega)=H(\omega) \tilde{E}(\omega)
$$

This set-up can be arbitrarily complex, containing many beam-splitters, interferometers or even several pulse shapers, its output can always be written as in equation 48 , the complex transfer function gathering all the subtleties of the set-up. Now the best we can do is to measure the output pulse $\tilde{E}_{\text {out }}(\omega)$. For that purpose, we don't have a lot of possible detectors: either slow photodiodes or spectrometers can be used. We will continue our discussion using a spectrometer but the same conclusion can be drawn using a photodiode. The spectrometer gives access to the spectral intensity:

$$
I_{\text {out }}(\omega)=\left|\tilde{E}_{\text {out }}(\omega)\right|^{2}=|H(\omega)|^{2}|\tilde{E}(\omega)|^{2}=|H(\omega)|^{2} A^{2}(\omega)
$$

As we can see, all the information on the spectral phase $\varphi(\omega)$ has disappeared from the expression of the signal we measure. The only information on the pulse we can gain from this type of measurement concerns the spectral amplitude $A(\omega)$. This explains what we have seen in the section 4.3 on referenced measurements: the reference was not needed to measure the spectral amplitude but was necessary to retrieve the spectral phase using linear optics. In conclusion of this quick try, the spectral phase of a short pulse can not be measured using stationary linear optics, unless we use a fully characterized reference 

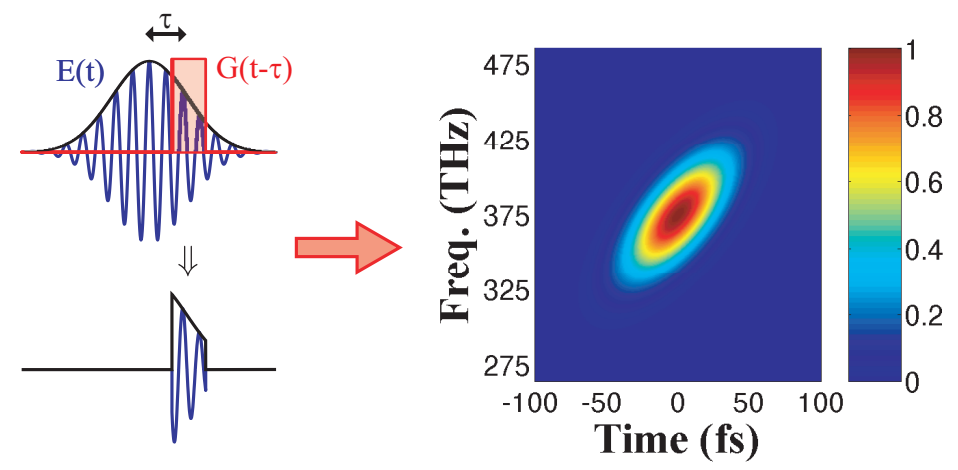

Figure 25. Principle of Frequency Optical Gating : The test pulse $E(t)$ is temporally sliced (or gated). The spectrum of this gated pulse is measured as a function of the delay $\tau$ between the gate $G(t)$ and the test pulse.

[135]. We thus need to use non-linear or non-stationary optics to achieve self-referenced complete characterization of a short pulse. This is a necessary but not a sufficient condition to get a complete measurement $\oint$.

\subsubsection{FROG and other variants}

The most ancient technique for self-referenced complete characterization is called Frequency Resolved Optical Gating, or FROG [136, 25]. The main idea in FROG is to temporally slice (or gate) the test pulse $E(t)$ and measure the spectrum of this gated pulse as a function of the delay $\tau$ between the gate $G(t)$ and the test pulse, as depicted on figure 25.

This gives a $2 \mathrm{D}$ signal

$$
S(\tau, \omega) \propto\left|\int E(t) G(t-\tau) e^{i \omega t} d t\right|^{2}
$$

that corresponds to a spectrogram of the test pulse (see equation 8 in section 2.2). From this 2D map, one can extract the spectral phase and amplitude of the test pulse using an iterative algorithm $[136,137,138]$. It is worth noting that extracting the pulse information from $S(\tau, \omega)$ corresponds to the well-known 2-D phase retrieval problem that can be found in many different fields. Moreover, the complete characterization of the test pulse remains possible even if the gate $G(t)$ is not fully characterized or even completely uncharacterized. There exist various implementations of FROG and they mostly differ by the way the gate $G(t)$ is generated. What all these implementations have in common is that they always require a non-linear interaction to generate the gate.

\section{$P G F R O G$}

One of the very first implementation was the Polarization Gating FROG [137] where

$\S$ In particular, both autocorrelation and cross-correlation contain a non-linear crystal without allowing a complete measurement of the test pulse. 


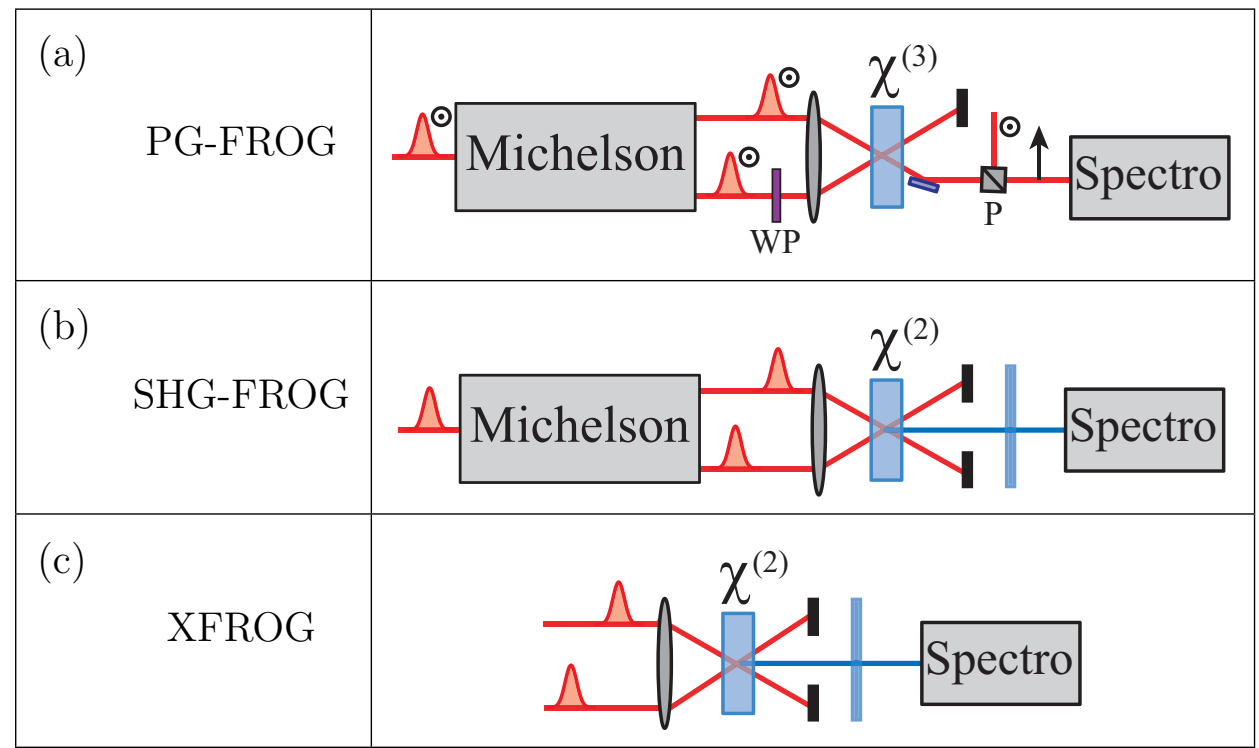

Figure 26. Schematic set-up of various versions of FROG techniques. (a) Polarization gating FROG, (b) Second harmonic generation FROG and (c) cross-correlation FROG. WP is a wave plate to tune the polarization, $\mathrm{P}$ is a polarizer, $\chi^{(2)}$ and $\chi^{(3)}$, non-linear susceptibilities.

the gating is implemented using the interaction between two copies of the test pulse $E(t)$ in a $\chi^{(3)}$ medium, as shown on figure 26 (a). In this particular implementation, a Kerr optical-gate is used and it results in a gate function following the intensity profile of the pulse $G(t)=|E(t)|^{2}$. With PG-FROG and for a reasonably simple test pulse, $S(\tau, \omega)$ is a fairly intuitive spectrogram. Some key features from the test pulse (like for example the presence of chirp and its sign) can thus be grasped directly by looking at $S(\tau, \omega)$ without recurring to the iterative algorithm.

\section{SHG FROG}

The most widely used FROG implementation is the Second Harmonic Generation (SHG) FROG[139] and its derivatives [137, 140]. One of the most widespread SHG FROG is the so called GRENOUILLE [140], a single-shot spectrally resolved autocorelation. As depicted on figure 26 (b), it is more easily implemented than PGFROG as it is just an intensimetric autocorrelator where the photodiode has been replaced by a spectrometer. Now, the gate is just a time delayed replica of the test pulse $G(t-\tau)=E(t-\tau)$ and the signal reads:

$$
S(\tau, \omega) \propto\left|\int E(t) E(t-\tau) e^{i \omega t} d t\right|^{2}
$$

Albeit easier to implement than PG-FROG, SHG-based FROG presents two disadvantages. First, the 2D signal is not as intuitive as the PG-FROG one. Second, as can be seen in equation 51, this signal is symmetric in time which means that there is a 
time ambiguity and the algorithm can retrieve either $E(t)$ or $E(-t)$. One should keep this in mind when trying to compensate for chirp or to get rid of pre- or post-pulses.

\section{XFROG}

The last FROG implementation we will present here, is the so-called XFROG [141] combined with the fast iterative algorithm called PCGPA [138]. This combination is particularly suited for the characterization of strongly shaped pulses or for the simultaneous characterization of pump and probe pulses in coherent control experiments. XFROG is a spectrally resolved cross-correlation between two pulses, as shown on figure 26 (c). Its signal takes the form

$$
S(\tau, \omega) \propto\left|\int E(t) E_{G}(t-\tau) e^{i \omega t} d t\right|^{2}
$$

where $E_{G}$ denotes the gate pulse. XFROG thus posses all the advantages of intensimetric cross-correlation: interferometric stability is not required for the delay between pulses, both pulses can lie in different spectral regions. It also have the ability to fully characterize simultaneously both test and gate pulses when used with PCGPA [138].

Moreover, if the gate pulse $E_{G}(t)$ is short compare to the test pulse $E(t)$, the signal $S(\tau, \omega)$ is an extremely intuitive spectrogram of the test pulse (see figure 3). XFROG is particularly suited to characterize the output of a pulse shaper, using a portion of the input pulse as the gate pulse. However, in many cases, XFROG is also used as a referenced measurement $[142,143]$ by using a fully characterized gate pulse, as it leads to better convergence of the retrieval algorithm. This can be useful to characterize the output of a microstructured fibre, as shown on figure 27.

Even when used as a referenced measurement, XFROG suffers from the same limitation than cross-correlation: it cannot retrieve the relative phase of non-overlapping pulses of a pulse sequence.

\section{Closing words on FROG}

FROG is a powerful technique for self-referenced complete characterization of ultrashort pulses. Many variants of FROG exist [136, 137, 145, 138, 25, 141, 140, 146], (see also table 1 in [12]). The table 4 lists some achievements using various variants of FROG. Some of these variants give an intuitive signal that trained user can partially interpret without recurring to an iterative algorithm. Moreover, FROG devices are relatively easy to implement and existing setups (like autocorrelator or a crosscorrelation) can be adapted to measure FROG traces.

The main drawback of FROG lies in the 2D nature of the signal and the iterative algorithm used to analyze it. First, the sampling and the covered range for both time and frequency axis have to be carefully chosen in order to reconstruct the test pulse with a good fidelity. In particular, as time and frequency are conjugate variables, the settings are not independent. Second, as all the information concerning both spectral phase and amplitude are encoded in the slow variation in intensity of $S(\tau, \omega)$, the camera used 


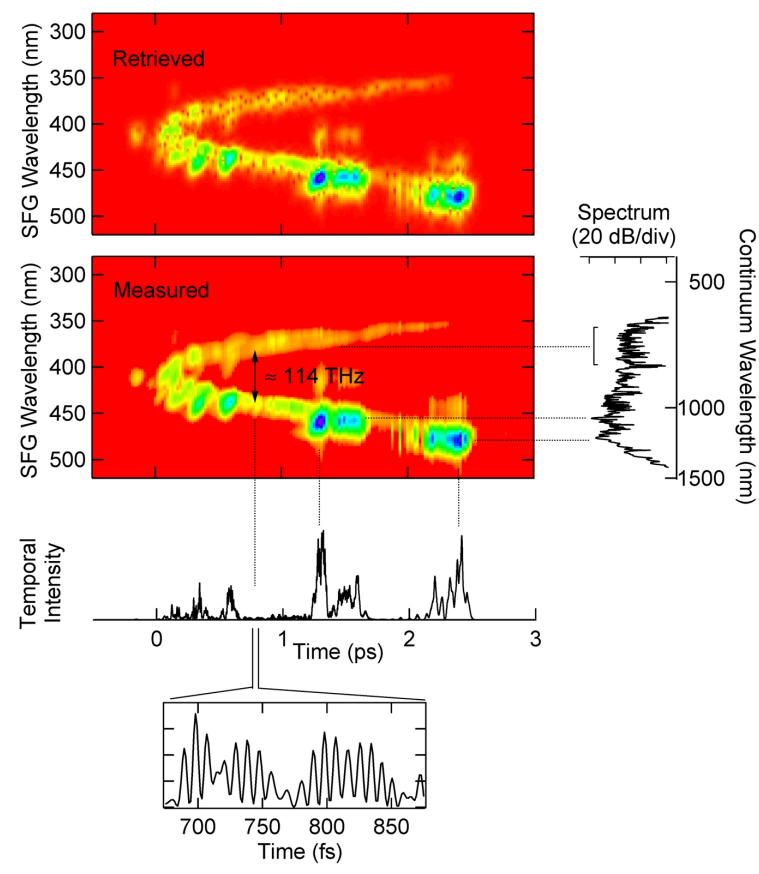

Figure 27. Taken from [144] : referenced XFROG Trace and retrieved temporal intensity of a supercontinuum out of microstructured fibre using the input of the fibre as a reference.

for recording the signal should exhibit both a high dynamic range and a high linearity. Last, the number of data points to record and analyze grows quadratically with the pulse complexity $\eta$. It means that for complex pulses, the time needed for the iterative algorithm to converge increases dramatically.

\begin{tabular}{|c|c|c|c|c|c|}
\hline \multirow{2}{*}{$\begin{array}{c}\text { Method } \\
\text { name }\end{array}$} & \multirow[b]{2}{*}{ Ref } & \multicolumn{3}{|c|}{ Source parameter } & \multirow[b]{2}{*}{ Main characteristics } \\
\hline & & $\begin{array}{c}\lambda_{0} \\
(\mathrm{~nm})\end{array}$ & $\begin{array}{l}\Delta \mathrm{t}(\mathrm{fs}) / \\
\Delta \lambda(\mathrm{nm})\end{array}$ & $\begin{array}{l}\mathrm{E}(\mathrm{nJ}) / \\
\mathrm{P}(\mu \mathrm{W})\end{array}$ & \\
\hline THG-FROG & {$[145]$} & 800 & $\simeq 100 / \mathrm{NA}$ & $3 / 3.10^{5}$ & $\begin{array}{l}\text { FROG using surface THG. No time } \\
\text { reversal ambiguity. }\end{array}$ \\
\hline PG-FROG & {$[137]$} & 620 & $\simeq 100 / \mathrm{NA}$ & $\simeq 10^{4} / \mathrm{NA}$ & $\begin{array}{l}\text { Original FROG using third order } \\
\text { non-linearity for polarization gating. } \\
\text { Intuitive trace, but requires energetic } \\
\text { pulses. }\end{array}$ \\
\hline \multirow[t]{2}{*}{ SD-FROG } & {$[136]$} & 620 & $\simeq 100 / \mathrm{NA}$ & $\simeq 10^{4} / \mathrm{NA}$ & \multirow[t]{2}{*}{$\begin{array}{l}\text { FROG using Self Diffraction as a non- } \\
\text { linear step. }\end{array}$} \\
\hline & [139] & 800 & $\simeq 70 / 24$ & $\mathrm{NA} / \mathrm{NA}$ & \\
\hline SHG-FROG & {$[147]$} & 800 & $\simeq 90 / \mathrm{NA}$ & $2 / \mathrm{NA}$ & $\begin{array}{l}\text { Spectrally resolved Second order non- } \\
\text { collinear autocorrelation. Easy to set- } \\
\text { up but time reversal and relative phase } \\
\text { ambiguities }\end{array}$ \\
\hline
\end{tabular}




\begin{tabular}{|c|c|c|c|c|c|}
\hline \multirow{2}{*}{$\begin{array}{l}\text { Method } \\
\text { name }\end{array}$} & \multirow[b]{2}{*}{ Ref } & \multicolumn{3}{|c|}{ Source parameter } & \multirow[b]{2}{*}{ Main characteristics } \\
\hline & & $\begin{array}{c}\lambda_{0} \\
(\mathrm{~nm})\end{array}$ & $\begin{array}{l}\Delta \mathrm{t}(\mathrm{fs}) / \\
\Delta \lambda(\mathrm{nm})\end{array}$ & $\begin{array}{l}\mathrm{E}(\mathrm{nJ}) / \\
\mathrm{P}(\mu \mathrm{W})\end{array}$ & \\
\hline & [148] & 1550 & $\simeq 40 / 150$ & $\mathrm{NA} / 5.10^{3}$ & \\
\hline & [149] & 5000 & $\simeq 700 / \mathrm{NA}$ & $100 / \mathrm{NA}$ & \\
\hline IFROG & {$[150]$} & 800 & $7 / 300$ & $\mathrm{NA} / \mathrm{NA}$ & $\begin{array}{l}\text { SHG-FROG but in collinear arrange- } \\
\text { ment. No blurring of the FROG trace } \\
\text { but fringes have to be resolved. }\end{array}$ \\
\hline GRENOUILLE & [140] & 800 & $\begin{array}{l}\simeq 100 \quad / \\
\simeq 20\end{array}$ & $\mathrm{NA} / \mathrm{NA}$ & $\begin{array}{l}\text { Simplified SHG-FROG using bi-prism } \\
\text { and thick SHG crystal. Time reversal } \\
\text { ambiguity. }\end{array}$ \\
\hline XFROG & {$[141]$} & 4000 & $\mathrm{NA} / \simeq 100$ & $\mathrm{NA} / 10^{3}$ & $\begin{array}{l}\text { Spectrally resolved cross-correlation } \\
\text { between two unknown pulses. Each } \\
\text { pulse can be in different spectral re- } \\
\text { gions. Both pulses are retrieved. }\end{array}$ \\
\hline Referenced & [143] & $\begin{array}{l}3000 \\
\text { to } \\
11000\end{array}$ & $\begin{array}{l}\simeq 100 / \\
\simeq 200\end{array}$ & $\simeq 3.10^{3} /$ & $\begin{array}{l}\text { XFROG with one unknown mid-IR } \\
\text { pulse and a fully referenced IR pulse. } \\
\text { A reference is needed. }\end{array}$ \\
\hline XFROG & {$[142]$} & 400 & $\simeq 170 / \mathrm{NA}$ & $\begin{array}{l}\simeq 6.10^{-2} / \\
\mathrm{NA}\end{array}$ & $\begin{array}{l}\text { XFROG using Down Conversion be- } \\
\text { tween one unknown UV pulse and a } \\
\text { fully referenced IR pulse. A reference } \\
\text { is needed. }\end{array}$ \\
\hline \multirow{3}{*}{ TG-FROG } & {$[151]$} & 426 & $\begin{array}{l}\simeq 250 \quad / \\
\simeq 1.5\end{array}$ & $200 / 200$ & $\begin{array}{l}\text { FROG using transient grating. } \\
\text { Adapted to UV pulses }\end{array}$ \\
\hline & {$[152]$} & 400 & $130 / 2.5$ & $\begin{array}{l}85.10^{3} \\
85.10^{3}\end{array}$ & \multirow[t]{2}{*}{$\begin{array}{l}\text { FROG using transient grating and a } \\
\text { simplified set-up like GRENOUILLE. }\end{array}$} \\
\hline & {$[152]$} & 800 & $150 / 8$ & $5.10^{5} / 5.10^{5}$ & \\
\hline
\end{tabular}

Table 4: List of some of the experimental achievements using variants of FROG: for the test pulse $\lambda_{0}$ is the central wavelength, $\Delta t$ the duration, $\Delta \lambda$ the spectral bandwidth, $E$ the smallest energy per pulse, $P$ the smallest average power.

\subsubsection{SPIDER and other variants}

There exists another method for self-referenced complete characterization that was developed a decade ago: Spectral Phase Interferometry for Direct Electric-field Reconstruction, or SPIDER [153] for short. We will present here the basic idea behind this technique along with some of its incarnations. This is by no mean an exhaustive review and for more details on all the different versions of SPIDER, interested readers 
should look at [154] and references therein. As its name suggests, SPIDER is based on spectral interferometry, with a special trick to allow for a self-referenced measurement. As we have described in section 4.3.1, when we apply spectral interferometry to a test pulse $E(t)$, using a reference $E_{r e f}(t-\tau)$, we retrieve both the spectral amplitude $A(\omega)$ of the test pulse and the quantity $\theta(\omega)=\varphi(\omega)-\varphi_{\text {ref }}(\omega)-\omega \tau$ (see equation 45) which is the phase difference between the test and reference pulses, modulo a linear term $-\omega \tau$ corresponding to the delay between the pulses.

The trick in SPIDER, that turns spectral interferometry (by essence a referenced measurement) into a self-referenced measurement, is to use the test pulse itself as a reference. More precisely, we use as a reference a copy of the test pulse that is shifted both temporally and spectrally so that spectral interferometry now yields both $A(\omega)$ and

$$
\theta(\omega)=\varphi(\omega)-\varphi(\omega-\Omega)-\omega \tau
$$

where $\Omega$ is the spectral shift (or spectral shear) and $\tau$ the temporal shift. If $\tau$ and $\Omega$ are known, we can derive from equation 53 the following quantity

$$
\frac{\varphi(\omega)-\varphi(\omega-\Omega)}{\Omega} \approx \frac{\partial \varphi}{\partial \omega}
$$

which is the phase gradient of the test pulse. From this gradient, we can reconstruct the phase either by concatenation or integration. The retrieved phase is the spectral phase of the test pulse, modulo a linear term: as already pointed out in section 2.1, this linear phase just corresponds to a delay and does not affect the shape of the pulse (that only depends on non-linear phase terms). SPIDER thus allows retrieving the spectral phase $\varphi(\omega)$ of the test pulse together with its spectral amplitude $A(\omega)$ using only simple, non-iterative algorithm: Fourier filtering to extract $\theta(\omega)$ and additions, multiplications and integration to derive $\varphi(\omega)$.

The conditions to fulfill for a meaningful measurement concern both the temporal shift $\tau$ and spectral shear $\Omega$. The conditions for $\tau$ are exactly the same than for spectral interferometry, as described in section 4.3.1: $\tau$ should be small enough for the spectral fringes to be correctly sampled by the spectrometer and $\tau$ should be longer than the duration of the pulse for the Fourier filtering to work. The spectral shear $\Omega$ should be small enough to fulfill the Whittaker Shannon theorem or in other words, to ensure that the sampling of $\varphi(\omega)$ is small enough so that no information was lost in the process. In theory any shear that respects $\Omega \leq 2 \pi / T$, where $T$ is the time interval where the pulse is non-zero, will produce an exact reconstruction of the spectral phase and amplitude. However, $\Omega$ should not be too small to avoid introducing unwanted noise in the concatenation process. In practice, a shear $\Omega$ in the order of a tenth of the spectral bandwidth of the pulse works well enough.

So far we haven't explained how the spectral shear $\Omega$ is generated. This is where a non-linear or non-stationary stage is involved. Indeed, as shown on figure 28 (a), the sum frequency mixing between our test pulse $\tilde{E}(\omega)$ and a quasi-monochromatic beam 


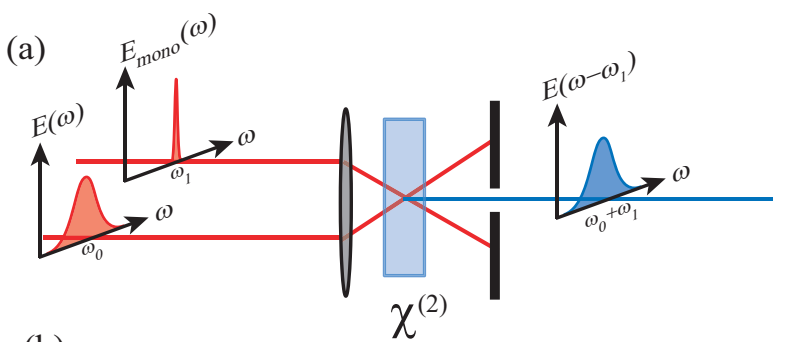

(b)

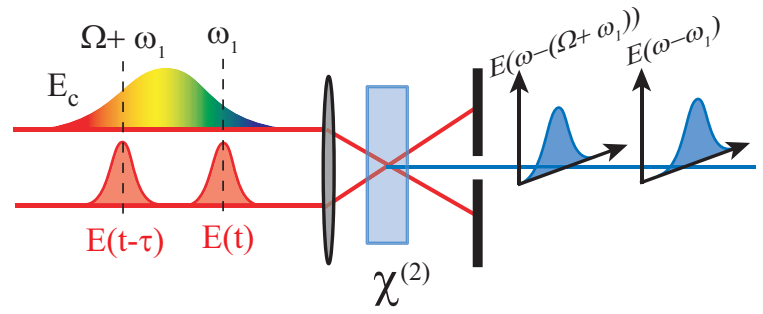

Figure 28. Spectral shearing by upconversion: (a) the upconversion between $E(\omega)$ a quasi-monochromatic pulse $E_{\text {mono }}(\omega)$ centered around $\omega_{1}$ generates a spectrally shifted replica $E\left(\omega-\omega_{1}\right)$; (b) the upconversion between two delayed replicas $E(t)$ and $E(t-\tau)$ and a chirped pulse $E_{c}(t)$ leads to two delayed and spectrally shifted replicas.

of center frequency $\omega_{1}$ gives $\tilde{E}\left(\omega-\omega_{1}\right)$, which is a spectrally shifted copy of the test pulse. In practice however, this introduces a spectral shift that is two to three orders of magnitude too high for SPIDER and that is not easily tunable. To circumvent this problem, the set-up presented on figure $28(\mathrm{~b})$ is used: each time delayed replica of the test pulse $E(t)$ and $E(t-\tau)$ are sum frequency mixed in a non-linear crystal with a highly chirped pulse $E_{C}(t)$. This chirped pulse acts as a quasi-monochromatic pulse which frequency changes slightly over time $\tau: \omega_{C}(t)=\omega_{1}+t / \varphi_{0}^{(2)}$. Each delayed replica is thus shifted in frequency by a different amount and the difference in frequency shift is $\Omega=\tau / \varphi_{0}^{(2)}$. By adjusting independently the chirp $\varphi_{0}^{(2)}$ and the delay $\tau$ between the replicas, one can set both $\Omega$ and $\tau$.

\section{The original flavour of SPIDER}

The original set-up for SPIDER is depicted in figure 29 (a). The test pulse is split into two time delayed replicas in a Michelson interferometer, which are upconverted with a highly chirped pulse in a non-linear crystal. The upconversion signal is then sent into the spectrometer and the spectral amplitude and phase are extracted from the interferogram using Fourier filtering. Usually, the highly chirped pulse is generated from the test pulse itself by taking a fraction of it and sending it through a double pass grating compressor. However, any chirped pulse that is coherent with the test one could do. As an example, for weak pulses, a Modified version of SPIDER (M-SPIDER) was developed [155] where the chirped pulse is taken inside the chirped pulse amplifier before recompression. This highly intense chirped pulse increases greatly the signal to noise ratio.

The original version of SPIDER is relatively simple and works well for pulses longer 


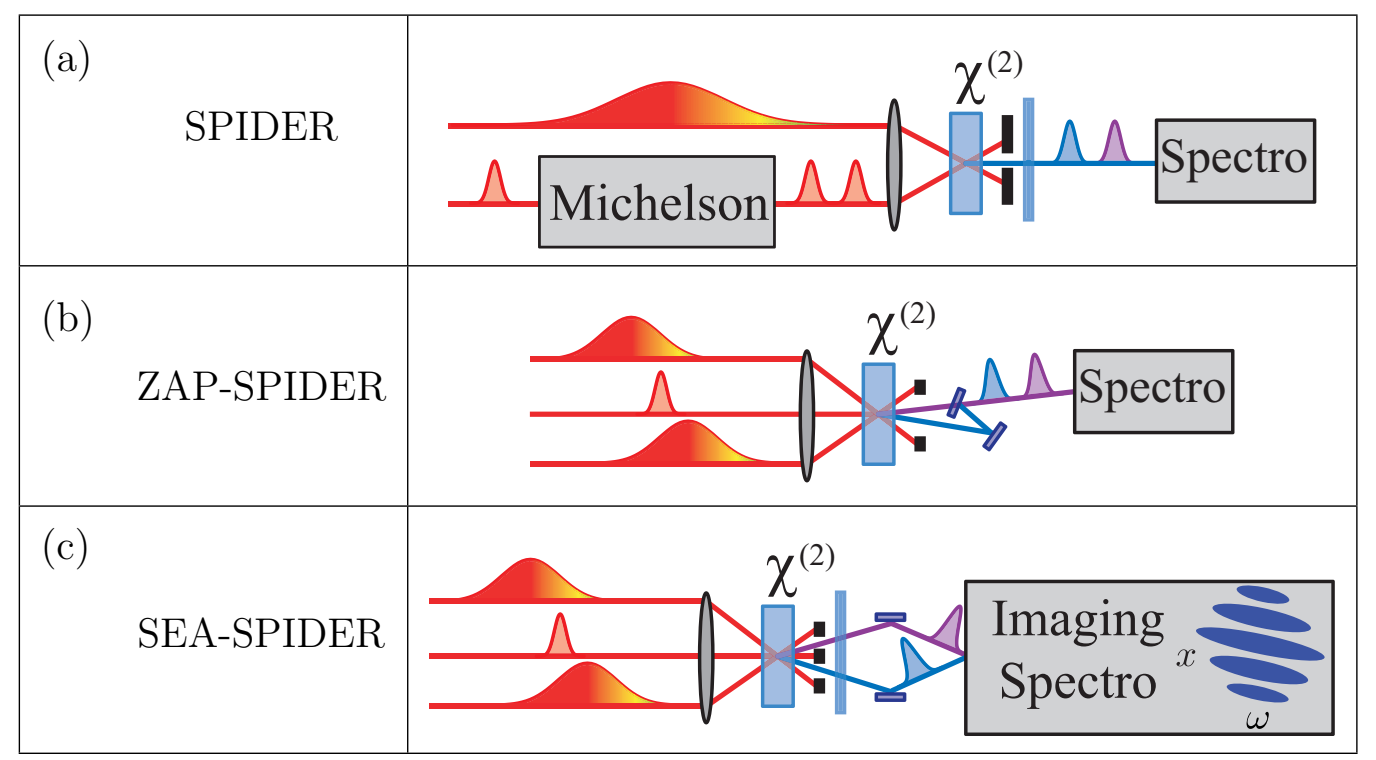

Figure 29. Different SPIDER techniques and their schematic setups. (a) Original SPIDER, (b) Zero Additional Phase SPIDER and (c) Spatially Encoded Arrangement SPIDER. $\chi^{(2)}$ denotes the non-linear susceptibility.

than few tens of femtosecond. The problem for shorter pulses arises from splitting the test pulse into two delayed replicas. Indeed, this implies going through some dispersive optics, like a beam splitter, which introduces unwanted dispersion. If the two replicas do not experience the exact same dispersion, the SPIDER measurement is degraded. However, using an optimized set-up for SPIDER[156], broadband pulses can be characterized by SPIDER, as shown on figure 30 .

Two different evolutions of SPIDER have been developed more specifically to characterize broadband and complex pulses. The first one, that we will not describe here, is Homodyne Optical Technique for SPIDER (HOT SPIDER) [157] that relies on a two-step measurement and is particularly suitable for short and weak pulses. The second approach is the Zero Additional Phase SPIDER (ZAP SPIDER) [158] that is described below.

\section{Zero Additional Phase SPIDER}

As its name suggests, in Zero Additional Phase SPIDER [158], the test pulse does not experience any distortion before entering the non-linear crystal. The untouched test pulse is upconverted with two time delayed replicas of a strongly chirped pulse, as shown on figure 29 (b). Because of the delay $\tau_{c}$ between the two chirped replicas, they exhibit different instantaneous frequency when being upconverted with the test pulse. As a result, two spectrally sheared replicas of the test pulse are generated. However, contrary to conventional SPIDER, these replicas are perfectly synchronized in time as they come from the very same test pulse. Moreover, as the two chirped pulses enter the crystal with different angles, the two sheared replicas propagate in different directions. The 


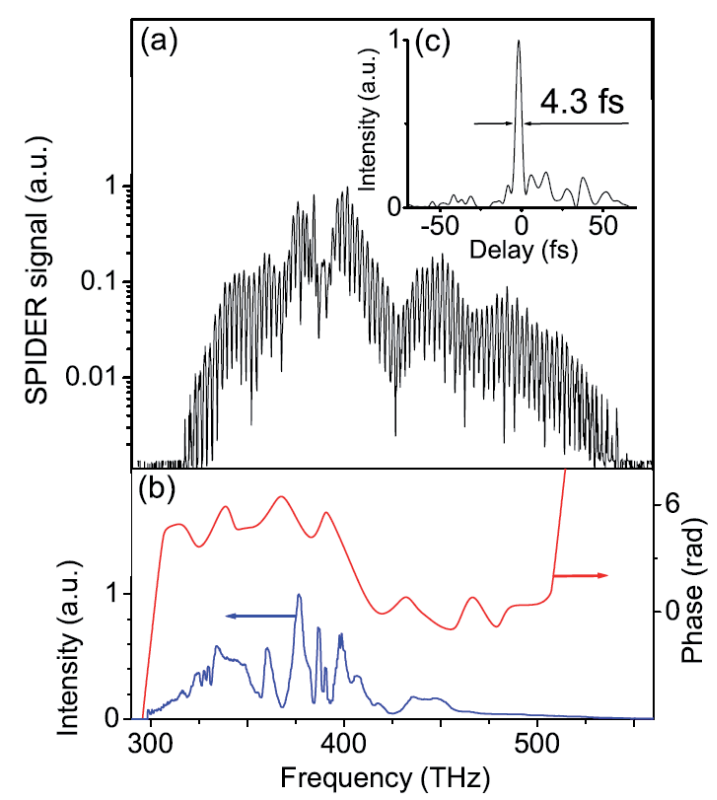

Figure 30. Taken from [156]: High Dynamic Range SPIDER measurement of a $4.3 \mathrm{fs}$ pulse out of a fibre compressor. (a) SPIDER interferogram, (b) reconstructed spectral phase and amplitude, (c) temporal intensity profile.

delay $\tau$ is independently set when recombining the sheared replicas before sending them into the spectrometer. The interferogram is similar to that of conventional SPIDER and the same analytical processing is performed. Besides the clear advantage of introduction virtually no distortion on the test pulse, another advantage of this arrangement is that the temporal delay $\tau$ and the shear $\Omega$ are completely independent: the former is set by delaying one of the upconverted replica before recombination whereas the latter can be set by changing the delay $\tau_{c}$ between the two chirped pulses. ZAP SPIDER has proved to be particularly efficient for shaped and weak pulses [159].

\section{Spatial Encoded Arrangement for SPIDER}

All the flavors of SPIDER we have presented so far, share in common the use of spectral fringes to encode the phase gradient. However, this is not the only option and the phase gradient can also be encoded in time. This is the case in time domain interferometry for SPIDER (no funny acronym here!) $[160,161]$ that was developed for spectral regions where spectrometers are not easily available.

Another possibility, is to encode the phase gradient in spatial fringes. This is the essence of Spatial Encoded Arrangement for SPIDER or SEA-SPIDER [162], particularly suited for broadband and shaped pulses. As one can see on figure 29 (c), the SEA-SPIDER set-up is close to the ZAP SPIDER we just presented. The main difference is that the two spectrally sheared replicas are kept synchronized in time and are recombined, with an angle, into an imaging spectrometer. This angle produces an ensemble of fringes along the vertical axis of the CCD (the wavelength being dispersed along the horizontal axis). More precisely, for a test pulse of the form $\tilde{E}(x, \omega)$ (where 
$x$ denotes the vertical coordinate), a spectral shear of $\Omega$, and for a difference between the transverse component of the propagation vectors $K$, one gets the following $2 \mathrm{D}$ interferogram:

$$
\begin{aligned}
I(x, \omega)= & |\tilde{E}(x, \omega)|^{2}+|\tilde{E}(x, \omega-\Omega)|^{2}+2|\tilde{E}(x, \omega)||\tilde{E}(x, \omega-\Omega)| \\
& \times \cos [\varphi(x, \omega)-\varphi(x, \omega-\Omega)+K x]
\end{aligned}
$$

The retrieval of the phase gradient is a slight modification of the conventional SPIDER algorithm: the 2D Fourier transform of $I(x, \omega)$ is composed of three lobes, all aligned along time 0 and centered around $+K, 0$ and $-K$. By filtering of the $+K$ sidelobe and inverse Fourier transforming, one gets

$$
\theta(x, \omega)=\varphi(x, \omega)-\varphi(x, \omega-\Omega)+K x
$$

from which one can retrieve $\varphi(x, \omega)$ by concatenation, provided $K$ is fully known. For a correct reconstruction, the conditions on the spectral shear $\Omega$ are the same then for conventional SPIDER, and the incidence difference $K$ is set so that there are few fringes across the beam (three fringes being a minimum). An imaging spectrometer with 128 pixel across the vertical axis is more then enough to properly sample the spatial fringes.

So SEA-SPIDER works in a similar way than normal SPIDER, but what are the advantages of this approach? First, SEA-SPIDER posses the same properties than ZAP-SPIDER. Moreover, as the fringes are not encoded along the frequency axis, the requirement on the spectral resolution of the spectrometer is less stringent. Indeed, in conventional SPIDER the spectral resolution has to be at least thrice better than the minimum required to fulfill the Whittaker Shannon theorem. This high requirement on resolution is difficult to fulfill when dealing with extremely broadband pulses which spectrum can span across several hundreds of nanometer. With SEA-SPIDER, the retrieval can be done at the sampling limit. SEA-SPIDER is thus the best SPIDER for shaped and broadband pulses.

Second advantage of SEA-SPIDER, the spectral phase and the spectral amplitude are reconstructed for every position $x$ along the vertical axis. This means that one can precisely measure spatio-temporal couplings like spatial chirp and pulse-front tilt or even more complex distortions. This is particularly useful for broadband or shaped pulses that often exhibit this kind of distortions.

Last advantage of SEA-SPIDER over other variants of SPIDER, the raw interferogram is intuitive: any deviation from perfectly straight horizontal fringes can be easily interpreted, as one can see on figure 31: a tilt corresponds to a $\varphi_{0}^{(2)}$ (figure $31(\mathrm{a})$, and (c)), a curvature to a $\varphi_{0}^{(3)}$, and any high order phase translates into deviation from straight lines (figure $31(\mathrm{~b})$ ).

\section{Closing word on SPIDER}

SPIDER is a reliable method to measure short and complex pulses. It comes in many 


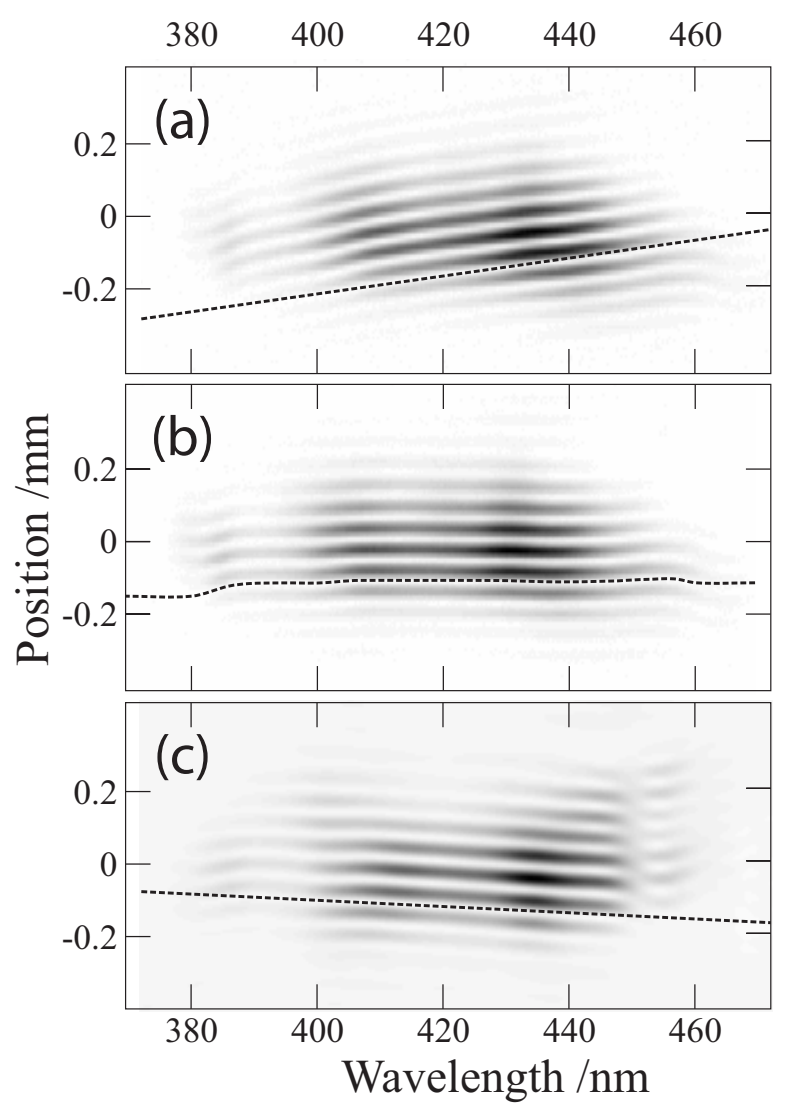

Figure 31. SEA SPIDER intuitive traces (courtesy of A. Wyatt): SEA SPIDER traces for a Femtolaser Rainbow oscillator, (a) for a positive $\varphi_{0}^{(2)}$, (b) for a recompressed pulse and (c) for a negative chirp. The small wiggles in (b) are a signature of small remaining high order phase.

flavors and the most up-to-date and exhaustive list can be found in references of [154]. Some experimental achievements using SPIDER and its variants are listed in table 5.

The two main advantages of SPIDER compared to competing techniques are, first, that it relies on a $1 \mathrm{D}$ signal and second that the phase retrieval algorithm is analytical and non-iterative. A 1D signal means that the amount of data to collect and treat is extremely small. Moreover, when using a 2D detector, like in an imaging spectrometer, one has an extra degree of freedom to play with. In SEA-SPIDER [162] for example, this allows for a simultaneous measurement of the spatio-temporal coupling. The noniterative Fourier transform algorithm allows for true real time retrieval of the spectral phase.

One strength of SPIDER is the encoding of the phase in fringes which allows for a good reconstruction of the phase even with poor dynamic of the CCD (down to 1 bit) and in the presence of strong noise [155].

The main drawback in SPIDER is that the calibration is particularly crucial and should not be overlooked. However, a new version of SPIDER [163] (Chirped ARrangement for SPIDER, CAR-SPIDER), uses the extra dimension to encode several 
configurations and provides a truly calibration-free measurement. Another limitation in SPIDER is the Fourier filtering technique (also known as the Takeda algorithm [133]) that is commonly used to extract the phase gradient from the interferogram. This filtering can be tricky to set and is the limiting factor in term of acceptable noise level for phase retrieval. A new algorithm based on fast wavelet analysis has recently been developed which address these two issues $[164,165]$.

\begin{tabular}{|c|c|c|c|c|c|}
\hline \multirow{2}{*}{$\begin{array}{l}\text { Method } \\
\text { name }\end{array}$} & \multirow[b]{2}{*}{ Ref } & \multicolumn{3}{|c|}{ Source parameter } & \multirow[b]{2}{*}{ Main characteristics } \\
\hline & & $\begin{array}{c}\lambda_{0} \\
(\mathrm{~nm})\end{array}$ & $\begin{array}{l}\Delta \mathrm{t}(\mathrm{fs}) / \\
\Delta \lambda(\mathrm{nm})\end{array}$ & $\begin{array}{l}\mathrm{E}(\mathrm{nJ}) / \\
\mathrm{P}(\mu \mathrm{W})\end{array}$ & \\
\hline \multirow{4}{*}{ SPIDER } & {$[153]$} & 800 & $\simeq 100 / \mathrm{NA}$ & $\begin{array}{ll}\text { few } & / \\
\text { few } & 10^{5}\end{array}$ & \multirow[t]{2}{*}{$\begin{array}{l}\text { Original SPIDER. Algebraic, non- } \\
\text { iterative reconstruction algorithm to } \\
\text { retrieve the phase gradient. }\end{array}$} \\
\hline & {$[166]$} & 800 & $\simeq 6 / 300$ & $3 / 3.10^{5}$ & \\
\hline & {$[167]$} & 800 & $\simeq 100 / \mathrm{NA}$ & $\mathrm{NA} / \mathrm{NA}$ & $\begin{array}{l}\text { Single shot SPIDER, with simultane- } \\
\text { ous and independent measurements of } \\
\text { spectral amplitude and spectral ampli- } \\
\text { tude. }\end{array}$ \\
\hline & {$[168]$} & 650 & $\simeq 4 / 500$ & $\mathrm{NA} / \mathrm{NA}$ & $\begin{array}{l}\text { SPIDER optimized for broadband } \\
\text { pulses. }\end{array}$ \\
\hline $\begin{array}{l}\text { High Dy- } \\
\text { namic Range } \\
\text { SPIDER }\end{array}$ & {$[156]$} & 850 & $\simeq 4.3 / 480$ & $2.10^{4} / 2.10^{4}$ & $\begin{array}{l}\text { SPIDER using heterodyne detection } \\
\text { based on chopper and fast 1-line CCD } \\
\text { camera. Designed for octave spanning. }\end{array}$ \\
\hline HOT SPIDER & {$[157]$} & 800 & $\simeq 100 / \mathrm{NA}$ & $250 / 250$ & $\begin{array}{l}\text { Two step measurement using intense } \\
\text { auxiliary pulse. Designed for low } \\
\text { average power/low intensity pulses. }\end{array}$ \\
\hline M SPIDER & {$[155]$} & 800 & $\simeq 100 / \mathrm{NA}$ & $3.6 / 3.6$ & $\begin{array}{l}\text { Original SPIDER but using an intense } \\
\text { auxiliary pulse from a Chirped Pulse } \\
\text { Amplifier. Designed for low average } \\
\text { power like fibre output. }\end{array}$ \\
\hline SPIDER (DC) & {$[169]$} & 410 & $\simeq 140 / 4$ & $70 / 70$ & $\begin{array}{l}\text { SPIDER using down-conversion as a } \\
\text { non-linear step. Designed for UV } \\
\text { pulses. }\end{array}$ \\
\hline \multirow[t]{2}{*}{$\begin{array}{l}\text { Time-domain } \\
\text { HOT SPIDER }\end{array}$} & {$[160]$} & 800 & $\simeq 100 / \mathrm{NA}$ & $\mathrm{NA} / \mathrm{NA}$ & $\begin{array}{l}\text { HOT SPIDER in time domain using } \\
\text { a shaper and a two-photon detector. } \\
\text { Compact, simple set-up without spec- } \\
\text { trometer. }\end{array}$ \\
\hline & {$[161]$} & 9200 & $\simeq 150 / \mathrm{NA}$ & $2.10^{3} / 2.10^{3}$ & $\begin{array}{l}\text { HOT SPIDER in time domain for Mid- } \\
\text { IR pulses. No spectrometer required, } \\
\text { designed for Mid-IR pulses. }\end{array}$ \\
\hline
\end{tabular}




\begin{tabular}{|c|c|c|c|c|c|}
\hline \multirow{2}{*}{$\begin{array}{l}\text { Method } \\
\text { name }\end{array}$} & \multirow[b]{2}{*}{ Ref } & \multicolumn{3}{|c|}{ Source parameter } & \multirow[b]{2}{*}{ Main characteristics } \\
\hline & & $\begin{array}{c}\lambda_{0} \\
(\mathrm{~nm}) \\
\end{array}$ & $\begin{array}{l}\Delta \mathrm{t}(\mathrm{fs}) / \\
\Delta \lambda(\mathrm{nm})\end{array}$ & $\begin{array}{l}\mathrm{E}(\mathrm{nJ}) / \\
\mathrm{P}(\mu \mathrm{W})\end{array}$ & \\
\hline \multirow[t]{2}{*}{$\begin{array}{l}\text { Electro-Optic } \\
\text { SPIDER }\end{array}$} & {$[170]$} & 1540 & $\begin{array}{l}750 \text { to } 30000 \\
/ \text { NA }\end{array}$ & $<10^{3} / \mathrm{NA}$ & \multirow[t]{2}{*}{$\begin{array}{l}\text { SPIDER using non-stationary step } \\
\text { (fast electro-optic phase modulation) } \\
\text { instead of non-linear step. Ultra- } \\
\text { sensitive, for telecom wavelengths. }\end{array}$} \\
\hline & {$[171]$} & 1570 & $200 / \mathrm{NA}$ & $5.10^{3} / \mathrm{NA}$ & \\
\hline \multirow[t]{4}{*}{ ZAP SPIDER } & [158] & 600 & $10 / 120$ & $\mathrm{NA} / \mathrm{NA}$ & $\begin{array}{l}\text { SPIDER without splitting of the test } \\
\text { pulse, using two auxiliary chirped } \\
\text { pulses. Designed for short pulses }(<20 \\
\text { fs). }\end{array}$ \\
\hline & {$[159]$} & 290 & $19 / \simeq 10$ & $10 / 10$ & \multirow[t]{2}{*}{$\begin{array}{l}\text { ZAP SPIDER using Down Conversion. } \\
\text { Designed for short UV pulses. }\end{array}$} \\
\hline & [159] & 290 & $7 / 40$ & $10 / 10$ & \\
\hline & {$[172]$} & 5100 & $100 / 400$ & $10^{3} / 10^{3}$ & ZAP SPIDER for Mid-IR pulses. \\
\hline HH SPIDER & {$[173]$} & 73 & $13 / 8$ & $\mathrm{NA} / \mathrm{NA}$ & $\begin{array}{l}\text { High Harmonic SPIDER using a double } \\
\text { seed scheme in High Harmonic Genera- } \\
\text { tion. Designed for XUV high harmon- } \\
\text { ics }\end{array}$ \\
\hline \multirow[t]{2}{*}{ SEA SPIDER } & {$[162]$} & 800 & $100 / \mathrm{NA}$ & $10 /$ few $10^{5}$ & \multirow[t]{2}{*}{$\begin{array}{l}\text { ZAP SPIDER but using spatial fringes. } \\
\text { Requires an imaging spectrometer. De- } \\
\text { signed for ultra broadband, modulated } \\
\text { pulses with spatiotemporal coupling. }\end{array}$} \\
\hline & {$[174]$} & 850 & $7 / 200$ & $\begin{array}{l}\simeq 4 \quad / \\
\simeq 3.10^{5}\end{array}$ & \\
\hline ARAIGNEE & {$[175]$} & $\begin{array}{l}740 \\
\text { to } \\
900\end{array}$ & $70 / \mathrm{NA}$ & $\mathrm{NA} / \simeq 10^{3}$ & $\begin{array}{l}\text { Simplified set-up using a thick SHG } \\
\text { crystal for spectral shearing. }\end{array}$ \\
\hline $\begin{array}{l}\text { TEA SPIDER } \\
(2 \mathrm{DSI})\end{array}$ & {$[176]$} & 800 & $5 / 400$ & $2 / 2.10^{5}$ & $\begin{array}{l}\text { SPIDER but using temporal fringes to } \\
\text { encode phase gradient. }\end{array}$ \\
\hline
\end{tabular}

Table 5: List of some of the experimental achievements using variants of SPIDER: for the test pulse $\lambda_{0}$ is the central wavelength, $\Delta t$ the duration, $\Delta \lambda$ the spectral bandwidth, $E$ the smallest energy per pulse, $P$ the smallest average power.

\subsubsection{Conclusion on characterization}

Many different characterization techniques are available, adapted to different pulse 
duration and wavelength. They range from the most simple and limited to more refined and complex ones. Implementing at least one complete characterization method is almost compulsory when using short and sometimes shaped pulses. However, incomplete and simple methods like auto- and cross-correlations should not be disregarded as they are easy to set up, fast and simple to use for daily check and also provide an independent measurement. Moreover, these methods are still actively developed and extended (see for example ref [94]). Concerning self-referenced techniques, we only presented here a small selection and tables 4 and 5 give some more details about other variants of both FROG and SPIDER.

As a conclusion on characterization of ultrashort pulses, we would like to give a personal view on the specific problem of characterizing strongly shaped pulses out of a pulse shaper. Theses pulses, because of their complexity (or high Time Bandwidth Product) can easily go beyond the limits of any given method. In particular, characterizing them with a self-referencing method (FROG, SPIDER or any variant of both) can be particularly difficult, requires special care and an optimized version of the method chosen for the task. Moreover, in order to properly and accurately generated this shaped output, the input pulse of the pulse shaper has to be decently characterized. For all these reasons, we believe that the most efficient approach is to combine a selfreferenced measurement of the input pulse and a referenced measurement of the output, using the input as a reference. As the input pulse is usually not really complex, any basic variant of FROG or SPIDER can be use for its measurement. For the referenced measurement, spectral interferometry or referenced XFROG are particularly well suited and easy to set up.

\section{The combination of pulse shaping and characterization techniques}

The last point we would like to mention in this tutorial is the recent development of characterization techniques that directly rely on a pulse shaper, an approach we decided to call "shaper assisted characterization".

One early precursor in this domain is STRUT (Spectrally and Temporally Resolved Up-conversion Techniques)[177] where a 4f-pulse shaper with a fixed slit is used to produce a spectrally narrowed replica of the test pulse (see $[178,177]$ for more details on the technique itself). However, in STRUT, the pulse shaper is not programmable and play less of a central role than in more recent implementations.

The key idea behind this approach is that most pulse shapers can be turned into versatile characterization devices by simply adding few optical elements that are usually already present in the lab. Indeed, a complete characterization technique can be implemented by combining three key ingredients: a linear stationary filter, a nonlinear optical process, and a slow detector as sketched on figure 32. In shaper assisted characterization techniques, a pulse shaper is used in order to implement the linear stationary filter, the rest of the set-up using already available elements (non-linear crystal, photodiode or spectrometer). In order to characterize the pulse entering the 
(a)

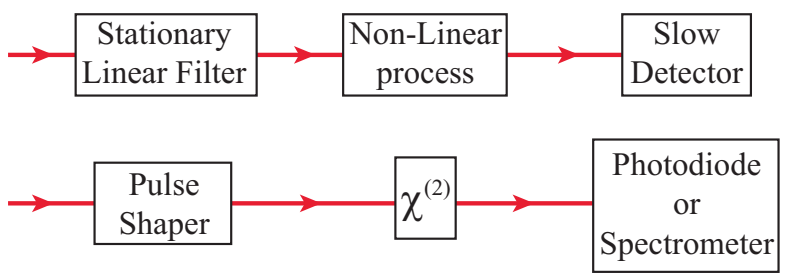

Figure 32. Generic set-up for complete characterization: (a) a pulse shaper serves as a generic versatile linear filter, followed by (b) a non-linear process which output is monitored by (c) a slow detector.

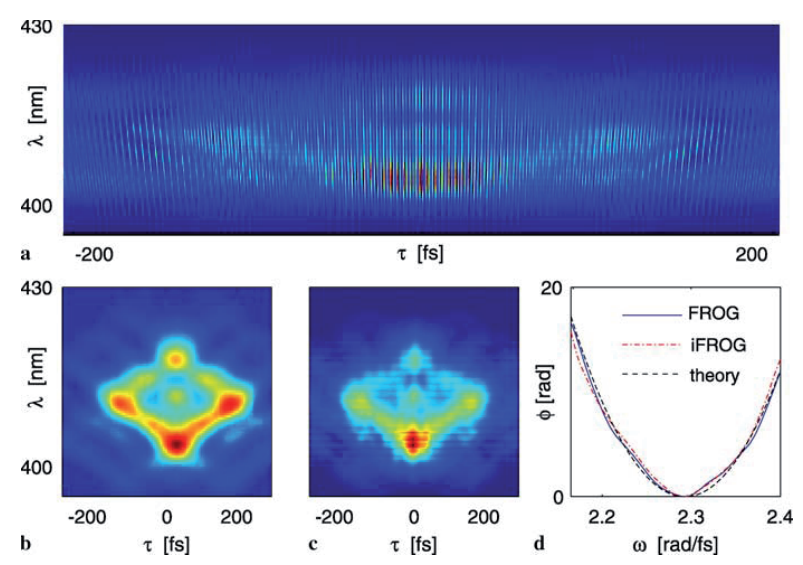

Figure 33. Taken from [178]. (a) iFROG measurements and (b) Reference FROG. (c) FROG trace retrieved from (a). (d) Extracted phases from the FROG and iFROG traces of a laser pulse passed through $5 \mathrm{~cm}$ of fused silica.

pulse shaper, the output of the non-linear process is recorded for a sequence of different waveforms. Depending on the sequence of waveforms used, many different techniques can be implemented. Indeed, variants of both FROG [179, 180, 178] and SPIDER $[160,181,180]$ have been implemented using this approach.

For instance FROG can be implemented by programming, using the shaper, delayed replica sent to a non-linear crystal $\left(\chi^{(2)}\right)$. Recording the spectra as a function of the delay gives an interferometric FROG (iFROG) trace. The classical FROG trace can be extracted by Fourier processing [178] (see Fig.33(c)) or obtained directly using calcite plate and type II crystal to avoid interferences [180]. Figure 33(a) taken in ref.[178] shows iFROG trace of a laser pulse passed through $5 \mathrm{~cm}$ of fused silica acquired using a 4f-line shaper. The oscillations are typical of interferometric traces. A reference FROG trace has been measured in Fig.33(b) for comparison whereas Fig.33(c) is the FROG trace extracted from (a). Finally the panel (d) shows the retrieved spectral phase for both iFROG and FROG techniques as well as theory.

What's more, using a pulse shaper opens the way to new possibilities that are not easily implemented using standard optics. This is the case of MIIPS (Multiphoton Intrapulse Interference Phase Scan) $[182,183,184]$ where the pulse shaper is used to add various known phase profiles to the test pulse. The output of the second-harmonic 
generation (SHG) is then spectrally resolved and recorded as a function of the added phase profiles.

It has been shown [184] that local maximum in the SHG spectra are observed when the total spectral phase is locally minimized. This occurs when its second derivative is equal to zero [184]:

$$
\varphi_{\text {tot }}^{(2)}\left(\omega_{m}\right)=\varphi^{(2)}\left(\omega_{m}\right)+f^{(2)}\left(\omega_{m}\right)=0
$$

where $f(\omega)$ is the added phase function and $\omega_{m}$ is the frequency corresponding to the maximum of the SHG spectrum. As $f^{(2)}(\omega)$ is a known function then one can determine $\varphi^{(2)}\left(\omega_{m}\right)$. By varying the applied function $f(\omega)$, one can retrieve $\varphi^{(2)}(\omega)$ across the whole spectrum and then reconstruct $\varphi(\omega)$ modulo a linear term. Figure 34 illustrates this principle using sets of quadratic phases as functions $f(\omega)$.

The solid line in the panel (a) represents the unknown $\varphi^{(2)}(\omega)$ which is mapped using an horizontal grid of function $f^{(2)}$ corresponding here to different amount of linear chirp. The intersections of the solid line with the horizontal lines fulfill equation 57 . In (b) the SHG spectrum is recorded and plotted as a function of $\omega$ for each function $f(\omega)$ : each maximum correspond to an intersection in (a) where the unknown phase is properly compensated for and thus measured. Plotting SHG intensity as a function of both chirp and frequency directly reveals the unknown phase $\varphi^{(2)}$ as shown in (c). Finally the number of functions $f(\omega)$ generable by a pulse shaper is extremely vast and leave the choice for appropriate set of functions, like sinusoidal phases[182].

One major advantage of all the shaper assisted characterization techniques is that the exact same set-up can be used to achieve several quasi-independent measurements of the same pulse, by simply changing the sequence of the waveforms used in order to implement different characterization techniques[179, 180, 178]. This constitute a unique advantage of shaper assisted characterization techniques over traditional ones where each technique relies on a dedicated experimental set-up.

One last advantage is that the resulting set-up is simple and straightforward as most of the complexity as been shifted to the pulse shaper and its programmed waveform. In particular, such a set-up does not involve any beam splitting or recombining and can thus be easily aligned and is compatible with in situ measurements.

Shaper assisted characterization techniques are thus particularly interesting whenever a pulse shaper is available. Indeed, any pulse shaper could do (both AOPDF and 4f-pulse shapers have been successfully used) and the overhead for turning it into a versatile characterization set-up is close to zero. However, theses techniques should be used wisely. Indeed, most of the implementations require a perfectly calibrated pulse shaper, used within its range of validity. Moreover various measurements made with the same pulse shaper are not truly independent and cannot fully replace an independent cross-check. 

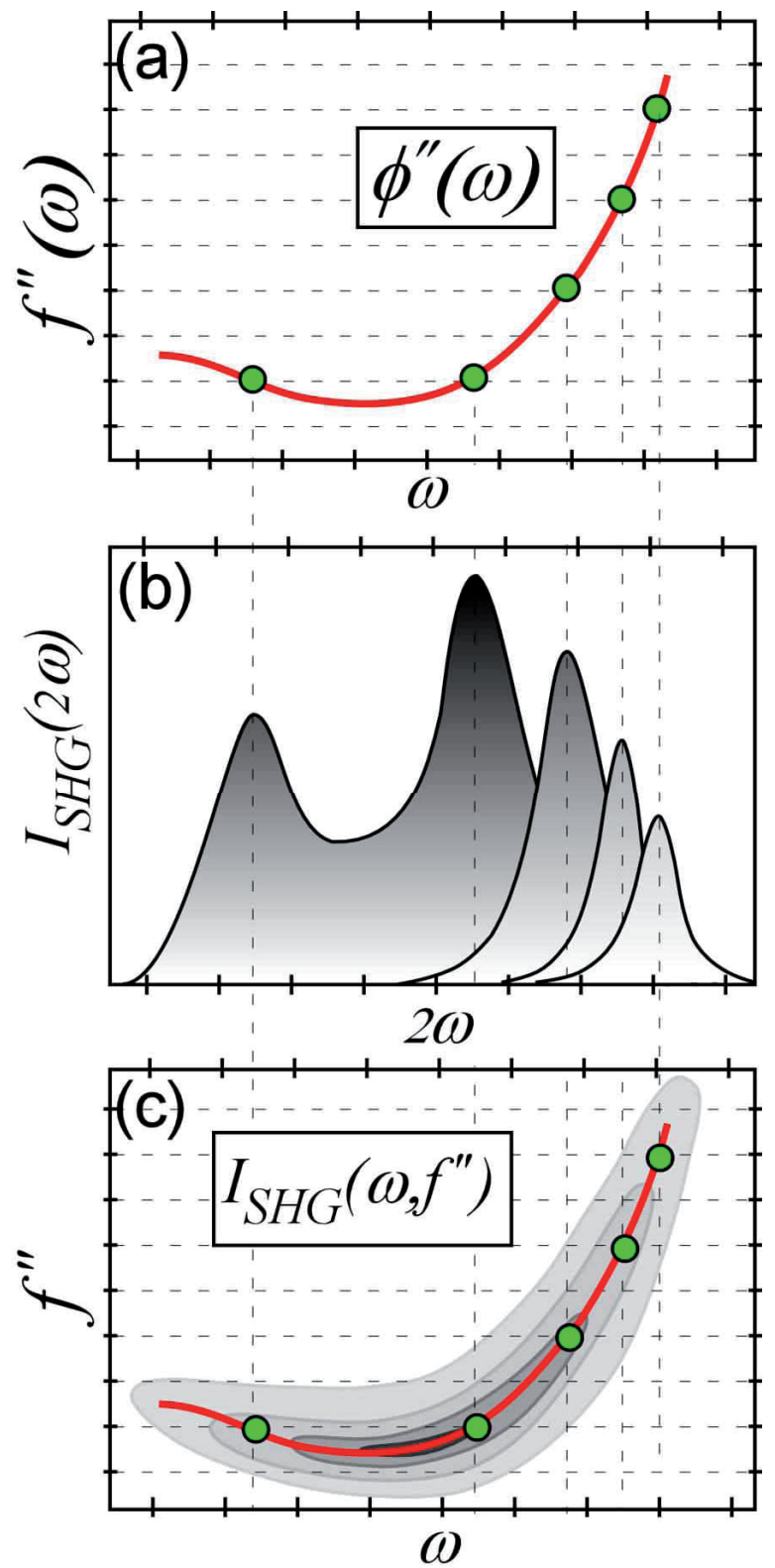

Figure 34. Taken from[185] Principle of the MIIPS method. (a) The unknown $\phi^{\prime \prime}(\omega)$ function is probed using a set of reference linear chirps represented by the horizontal grid. (b) The maximum SHG intensity for every frequency indicates that the corresponding reference chirp value compensates the unknown function at the position of the maximum. (c) A two-dimensional contour plot mapping the intensity of the SHG as a function of chirp and frequency directly reveals the unknown $\phi^{\prime \prime}(\omega)$. 


\section{General conclusion}

In this tutorial, we have tried to give an overview of some of the most widespread techniques in ultrashort pulse shaping and characterization. This tutorial is be no means a complete review but an introduction to two fast evolving fields of research. For any technique presented here, interested readers can find more details and in depth discussion in the provided references. As a general conclusion, we would like to give one last piece of advice for both shaping and characterization. In both cases, it is crucial to keep in mind the range of validity, or the range of usage, out of which the result is not well determined. This piece of advice sounds elementary, but as shapers and characterization techniques tend to grow more complex and more subtle, it is not always straightforward to fully apprehend their limits. This is particularly true for shaper assisted characterization techniques as one should stay within both the range of validity of the technique implemented and within the range of abilities of the shaper used.

\section{Bibliography}

[1] A. J. DeMaria, C. M. Ferrar, and Jr. G. E. Danielson. Mode locking of a nd[sup $3+$ ]-doped glass laser. Applied Physics Letters, 8(1):22-24, 1966.

[2] D. Goswami. Optical pulse shaping approaches to coherent control. Phys. Rep., 374(6):385, 2003.

[3] Kenji Ohmori. Wave-packet and coherent control dynamics. Annual Review of Physical Chemistry, 60(1):487-511, 2009.

[4] Marcos Dantus and Vadim V. Lozovoy. Experimental coherent laser control of physicochemical processes. Chemical Reviews, 104(4):1813-1859, 2004.

[5] S-H. Shim and M. T. Zanni. How to turn your pump-probe instrument into a multidimensional spectrometer: 2d ir and vis spectroscopies via pulse shaping. Phys. Chem. Chem. Phys., 11(5):748-761, February 2009.

[6] Yaron Silberberg. Quantum coherent control for nonlinear spectroscopy and microscopy. Annual Review of Physical Chemistry, 60(1):277-292, 2009.

[7] A. Efimov, M. D. Moores, B. Mei, J. L. Krause, C. W. Siders, and D. H. Reitze. Minimization of dispersion in an ultrafast chirped pulse amplifier using adaptive learning. Appl. Phys. B, 70(June):S133, 2000.

[8] S. Weber, B. Chatel, and B. Girard. Factoring numbers with interfering random waves. EuroPhysics Letters, 83:34008, 2008.

[9] Damien Bigourd, Beátrice Chatel, Bertrand Girard, and Wolfgang P. Schleich. Factorization of numbers with the temporal talbot effect: Optical implementation by a sequence of shaped ultrashort pulses. Phys. Rev. Lett., 100:030202, 2008.

[10] H. P. Sardesai, C. C. Chang, and A. M. Weiner. A femtosecond code-division multiple-access communication system test bed. J. Lightw. Technol., 16(11):1953, 1998.

[11] ***A. M. Weiner. Femtosecond pulse shaping using spatial light modulators. Rev. Sci. Instr., 71(5):1929-60, 2000.

[12] ${ }^{* * *}$ Rick Trebino, Kenneth W. DeLong, David N. Fittinghoff, John N. Sweetser, Marco A. Krumbügel, and Bruce A. Richman. Measuring ultrashort laser pulses in the time-frequency domain using frequency-resolved optical gating. Review of Scientific Instruments, 68(9):3277$3295,1997$. 
[13] ***Ian A. Walmsley and Christophe Dorrer. Characterization of ultrashort electromagnetic pulses. Adv. Opt. Photon., 1(2):308-437, 2009.

[14] **J.C. Diels and W. Rudolph. Ultrashort Laser Pulse Phenomena, 2nd edition. sept. 2006.

[15] **M. Wollenhaupt, A. Assion, and T. Baumert. Springer Handbook of Lasers and Optics, chapter 12, pages 937-983. Springer, 2007.

[16] P Hannaford. Femtosecond Laser Spectroscopy. 2005.

[17] Ferenc Krausz and Misha Ivanov. Attosecond physics. Reviews of Modern Physics, 81(1):163, 2009.

[18] B. Chatel, J. Degert, S. Stock, and B. Girard. Competition between sequential and direct paths in a two-photon transition. Phys. Rev. A, 68(4):041402, 2003.

[19] B. Broers, H. B. van Linden van den Heuvell, and L. D. Noordam. Efficient population transfer in a three-level ladder system by frequency-swept ultrashort laser pulses. Phys. Rev. Lett., 69:2062, 1992.

[20] B. Broers, J.F. Christian, J.H. Hoogenaard, W.J. van der Zande, H.B. van Linden van den Heuvell, and L.D. Noordam. Time-resolved dynamics of electronic wave packets above the classical field-ionisation threshold. Phys. Rev. Lett., 71(3):344, 1993.

[21] Cathie Ventalon, James M. Fraser, Marten H. Vos, Antigoni Alexandrou, Jean-Louis Martin, and Manuel Joffre. Coherent vibrational climbing in carboxyhemoglobin. Proc. Nat. Acad. Sc., 101(36):13216, 2004.

[22] B. Chatel, J. Degert, and B. Girard. Role of quadratic and cubic spectral phases in ladder climbing with ultrashort pulses. Phys. Rev. A, 70(5):053414, 2004.

[23] Leon Cohen. Time-frequency distributions-a review. Proceedings of the IEEE, 77(7):941, 1989.

[24] J Paye. The chronocyclic representation of ultrashort light pulses. I.E.E.E. Journal of Quantum Electronics, 28(10):2262-2273, 1992.

[25] ***R. Trebino. Frequency-Resolved Optical Gating: The Measurement of Ultrashort Laser Pulses. Kluwer Academic, 2000.

[26] Richard S. Judson and Herschel Rabitz. Teaching lasers to control molecules. Phys. Rev. Lett., 68(10):1500-3, 1992.

[27] Andrei C. Florean, David Cardoza, James L. White, J. K. Lanyi, Roseanne J. Sension, and Philip H. Bucksbaum. Control of retinal isomerization in bacteriorhodopsin in the highintensity regime. Proc. Nat. Acad. Sc., 106(27):10896-10900, 2009.

[28] Janne Savolainen, Riccardo Fanciulli, Niels Dijkhuizen, Ana L. Moore, Jürgen Hauer, Tiago Buckup, Marcus Motzkus, and Jennifer L. Herek. Controlling the efficiency of an artificial light-harvesting complex. Proc. Nat. Acad. Sc., 105(22):7641-7646, 2008.

[29] T Brixner, NH Damrauer, P Niklaus, and G Gerber. Photoselective adaptive femtosecond quantum control in the liquid phase. Nature, 414(6859):57-60, NOV 012001.

[30] RJ Levis, GM Menkir, and H Rabitz. Selective bond dissociation and rearrangement with optimally tailored, strong-field laser pulses. Science, 292(5517):709-713, APR 272001.

[31] Carsten Winterfeldt, Christian Spielmann, and Gustav Gerber. Colloquium: Optimal control of high-harmonic generation. Reviews of Modern Physics, 80(1):117, 2008.

[32] JM Dela Cruz, I Pastirk, M Comstock, VV Lozovoy, and M Dantus. Use of coherent control methods through scattering biological tissue to achieve functional imaging. Proc. Nat. Acad. Sc., 101(49):16996-17001, 2004.

[33] A. Assion, T. Baumert, M. Bergt, T. Brixner, B. Kiefer, V. Seyfried, M. Strehle, and G. Gerber. Control of chemical reactions by feedback-optimized phase-shaped femtosecond laser pulses. Science, 282:919, 1998.

[34] Patrick Nuernberger, Gerhard Vogt, Tobias Brixner, and Gustav Gerber. Femtosecond quantum control of molecular dynamics in the condensed phase. Phys. Chem. Chem. Phys., 9(20):2470$2497,2007$.

[35] A. Monmayrant, B. Chatel, and B. Girard. Atomic spirograph: measurement of the excited state wave function using coherent transients. Phys. Rev. Lett., 96:103002, 2006. 
[36] J. Degert, W. Wohlleben, B. Chatel, M. Motzkus, and B. Girard. Realization of a time-domain fresnel lens with coherent control. Phys. Rev. Lett., 89(20):203003, 2002.

[37] N. Dudovich, B. Dayan, S. M. Gallagher Faeder, and Y. Silberberg. Transform-limited pulses are not optimal for resonant multiphoton transitions. Phys. Rev. Lett., 86(1):47, 2001.

[38] N. Dudovich, D. Oron, and Y. Silberberg. Coherent transient enhancement of optically induced resonant transitions. Phys. Rev. Lett., 88(12):123004, 2002.

[39] Zohar Amitay, Andrey Gandman, Lev Chuntonov, and Leonid Rybak. Multichannel selective femtosecond coherent control based on symmetry properties. Phys. Rev. Lett., 100(19):193002, 2008.

[40] A. Pe'er, B. Dayan, A. A. Friesem, and Y. Silberberg. Temporal shaping of entangled photons. Phys. Rev. Lett., 94(7):073601-4, 2005.

[41] K. A. Walowicz, I. Pastirk, V. V. Lozovoy, and M. Dantus. Multiphoton intrapulse interference. i. control of multiphoton processes in condensed phases. J. Phys. Chem. A, 106(41):9369, 2002.

[42] T Martchenko, C Siedschlag, S Zamith, HG Muller, and MJJ Vrakking. Optimal control of femtosecond laser-cluster interactions. Phys. Rev. A, 72(5):053202, 2005.

[43] AF Bartelt, T Feurer, and L Woste. Understanding optimal control results by reducing the complexity. Chemical Physics, 318(3):207-216, 2005.

[44] N. Dudovich, T. Polack, A. Pe'er, and Y. Silberberg. Simple route to strong-field coherent control. Phys. Rev. Lett., 94(8):083002, 2005. 083002.

[45] Stephen D. Clow, Carlos Trallero-Herrero, Thomas Bergeman, and Thomas Weinacht. Strong field multiphoton inversion of a three-level system using shaped ultrafast laser pulses. Phys. Rev. Lett., 100(23):233603, 2008.

[46] T. Laarmann, I. Shchatsinin, P. Singh, N. Zhavoronkov, M. Gerhards, C. P. Schulz, and I. V. Hertel. Coherent control of bond breaking in amino acid complexes with tailored femtosecond pulses. The Journal of Chemical Physics, 127(20):201101, 2007.

[47] R. de Nalda, C. Horn, M. Wollenhaupt, M. Krug, L. Banares, and T. Baumert. Pulse shaping control of alignment dynamics in n-2. Journal of Raman Spectroscopy, 38(5):543-550, 2007.

[48] K. Ohmori, Y. Sato, E. E. Nikitin, and S. A. Rice. High-precision molecular wave-packet interferometry with hgar dimers. Phys. Rev. Lett., 91(24):243003, 2003.

[49] ***I. Walmsley, L. Waxer, and C. Dorrer. The role of dispersion in ultrafast optics. Rev. Sci. Instr., 72(1):1-29, 2001.

[50] E.B. Treacy. Optical pulse compression with diffraction gratings. I.E.E.E. Journal of Quantum Electronics, QE-5:454-458, 1969.

[51] O. E. Martinez. 3000 times grating compressor with positive group velocity dispersion: application to fiber compensation in 1.3-1.6 mu $\mathrm{m}$ region. IEEE Journal of Quantum Electronics, QE-23(1):59-64, 1987.

[52] R. Szipocs and A. Kohazi-Kis. Theory and design of chirped dielectric laser mirrors. Applied Physics B Lasers and Optics, 65:115, 1997.

[53] **Günter Steinmeyer. A review of ultrafast optics and optoelectronics. Journal of Optics A: Pure and Applied Optics, 5(1):R1, 2003.

[54] C. Froehly, B. Colombeau, and M. Vampouille. Shaping and analysis of picosecond light pulses. Progress in optics, 20:65-153, 1983.

[55] A. M. Weiner, J. P. Heritage, and E. M. Kirschner. High-resolution femtosecond pulse shaping. Journal of the Optical Society of America B (Optical Physics), 5(8):1563-72, 1988.

[56] *T. Brixner and G. Gerber. Femtosecond polarization pulse shaping. Optics Letters, 26(8):557-9, 2001.

[57] T. Feurer, Joshua C. Vaughan, Richard M. Koehl, and Keith A. Nelson. Multidimensional control of femtosecond pulses by use of a programmable liquid-crystal matrix. Optics Letters, 27(8):652-654, 2002.

[58] **M.B Danailov and I.P. Christov. Time-space shaping of light pulses by fourier optical processing. Journal of modern optics, 36(6):725-731, 1989. 
[59] O. Boyko, C. Valentin, G. Rey, L. Antonucci, Ph. Balcou, and S. Coudreau. Temporal superresolution of ultrashort laser pulses. Opt. Express, 13(20):8222-8230, 2005.

[60] *A. Monmayrant and B. Chatel. A new phase and amplitude high resolution pulse shaper. Rev. Sci. Instr., 75(8):2668-71, 2004.

[61] *S.H. Shim, D. B. Strasfeld, E.C. Fulmer, and M.T. Zanni. Femtosecond pulse shaping directly in the mid-ir using acousto-optic modulation. Opt. Lett., 31:838, 2006.

[62] Takasumi Tanabe, Hiroshi Tanabe, Yuichi Teramura, and Fumihiko Kannari. Spatiotemporal measurements based on spatial spectral interferometry for ultrashort optical pulses shaped by a fourier pulse shaper. J. Opt. Soc. Am. B, 19(11):2795-2802, 2002.

[63] **M. M. Wefers and K. A. Nelson. Analysis of programmable ultrashort waveform generation using liquid-crystal spatial light-modulators. Journal of the Optical Society of America B (Optical Physics), 12(7):1343-62, 1995.

$[64]{ }^{* *}$ C. Dorrer and F. Salin. Phase amplitude coupling in spectral phase modulation. IEEE J. Select. Top. Quantum Electron., 4(2):342, 1998.

[65] **B.J. Sussman, R. Lausten, and A. Stolow. Focusing of light following a 4-f pulse shaper: Considerations for quantum control. phys Rev A, 77:043416, 2008.

[66] F. Frei, A. Galler, and T. Feurer. Space-time coupling in femtosecond pulse shaping and its effects on coherent control. The Journal of Chemical Physics, 130(3):034302, 2009.

[67] Nirit Dudovich, Dan Oron, and Yaron Silberberg. Quantum control of the angular momentum distribution in multiphoton absorption processes. Phys. Rev. Lett., 92(10):103003, 2004.

[68] A. Efimov, C. Schaffer, and D. H. Reitze. Programmable shaping of ultrabroad-bandwidth pulses from a ti:sapphire laser. Journal of the Optical Society of America B (Optical Physics), 12(10):1968-80, 1995.

[69] D. Zeidler, T. Hornung, D. Proch, and M. Motzkus. Adaptive compression of tunable pulses from a non-collinear-type opa to below 16 fs by feedback-controlled pulse shaping. Applied Physics $B, 70$ (June):S125, 2000.

[70] H. Wang, Z. Zheng, D. E. Leaird, A. M. Weiner, T. A. Dorschner, J. J. Fijol, L. J. Friedman, H. Q. Nguyen, and L. A. Palmaccio. 20-fs pulse shaping with a 512-element phase-only liquid crystal modulator. IEEE J. Sel. Top. Quantum Electron., 7(4):718-27, 2001.

[71] D. Yelin, D. Meshulach, and Y. Silberberg. Adaptive femtosecond pulse compression. Opt. Lett., 22(23):1793-1795, 1997.

[72] D Sofikitis, S Weber, A Fioretti, R Horchani, M Allegrini, B Chatel, D Comparat, and P Pillet. Molecular vibrational cooling by optical pumping with shaped femtosecond pulses. New Journal of Physics, 11(5):055037, 2009.

[73] A. Mermillod-Blondin, C. Mauclair, A. Rosenfeld, J. Bonse, I. V. Hertel, E. Audouard, and R. Stoian. Size correction in ultrafast laser processing of fused silica by temporal pulse shaping. Applied Physics Letters, 93(2):021921, 2008.

[74] Joshua Vaughan, T. Feurer, Katherine Stone, and Keith Nelson. Analysis of replica pulses in femtosecond pulse shaping with pixelated devices. Optics Express, 14(3):1314, 2006.

[75] C. W. Hillegas, J. X. Tull, D. Goswami, D. Strickland, and W. S. Warren. Femtosecond laser pulse shaping by use of microsecond radio-frequency pulses. Opt. Lett., 19(10):737, 1994.

[76] **M. A. Dugan, J. X. Tull, and W. S. Warren. High-resolution acousto-optic shaping of unamplified and amplified femtosecond laser pulses. Journal of the Optical Society of America B (Optical Physics), 14(9):2348-58, 1997.

[77] I. C. Chang. I. acoustooptic devices and applications. Sonics and Ultrasonics, IEEE Transactions on, 23(1):2-21, 1976.

[78] M. Roth, M. Mehendale, A. Bartelt, and H. Rabitz. Acousto-optical shaping of ultraviolet femtosecond pulses. Applied Physics B: Lasers and Optics, 80(4 - 5):441, 2005.

[79] Matthew Fetterman, Debabrata Goswami, Dorine Keusters, Weiguo Yang, June-Koo Rhee, and Warren Warren. Ultrafast pulse shaping: amplification and characterization. Opt. Express, 3(10):366-375, 1998. 
[80] Yu Huang and Arthur Dogariu. Application of adaptive feedback loop for ultra-violet femtosecond pulse shaper control. Opt. Express, 14(21):10089-10094, 2006.

[81] C. Trallero-Herrero, J. L. Cohen, and T. C. Weinacht. Strong field atomic phase matching. Phys. Rev. Lett., 96:063603, 2006.

[82] Matthias Roth, Laurent Guyon, Jonathan Roslund, Veronique Boutou, Francois Courvoisier, Jean-Pierre Wolf, and Herschel Rabitz. Quantum control of tightly competitive product channels. Phys. Rev. Lett., 102(25):253001-4, 2009.

[83] M. Greenfield, S. D. McGrane, and D. S. Moore. Control of cis-stilbene photochemistry using shaped ultraviolet pulses. The Journal of Physical Chemistry A, 113(11):2333-2339, 2009.

[84] Marija Kotur, Thomas Weinacht, Brett J. Pearson, and Spiridoula Matsika. Closed-loop learning control of isomerization using shaped ultrafast laser pulses in the deep ultraviolet. The Journal of Chemical Physics, 130(13):134311, 2009.

[85] Sang-Hee Shim, David B. Strasfeld, and Martin T. Zanni. Generation and characterization of phase and amplitude shaped femtosecond mid-ir pulses. Opt. Express, 14(26):13120-13130, 2006.

[86] T. C. Weinacht, J. Ahn, and P. H. Bucksbaum. Measurement of the amplitude and phase of a sculpted rydberg wave packet. Phys. Rev. Lett., 80(25):5508, 1998.

[87] T. C. Weinacht, J. Ahn, and P. H. Bucksbaum. Controlling the shape of a quantum wavefunction. Nature, 397:233, 1999.

[88] Peifang Tian, Dorine Keusters, Yoshifumi Suzaki, and Warren S. Warren. Femtosecond phasecoherent two-dimensional spectroscopy. Science, 300(5625):1553-1555, 2003.

[89] B. Pearson and T.C. Weinacht. Shaped ultrafast laser pulses in the deep ultraviolet. optics express, 17(7):4385, 2007.

[90] E. Zeek, K. Maginnis, S. Backus, U. Russek, M. Murnane, G. Mourou, H. Kapteyn, and G. Vdovin. Pulse compression by use of deformable mirrors. Optics Letters, 24(7):493-495, 1999.

[91] Jesus Gardu no Mejía, Alan H. Greenaway, and Derryck T. Reid. Programmable spectral phase control of femtosecond pulses by use of adaptive optics and real-time pulse measurement. $J$. Opt. Soc. Am. B, 21(4):833-843, 2004.

[92] M. Hacker, G. Stobrawa, R. Sauerbrey, T. Buckup, M. Motzkus, M. Wildenhain, and A. Gehner. Micromirror slm for femtosecond pulse shaping in the ultraviolet. Applied Physics B-Lasers And Optics, 76(6):711-714, 2003.

[93] A. Rondi, J. Extermann, L. Bonacina, S. Weber, and J.-P. Wolf. Characterization of a memsbased pulse-shaping device in the deep ultraviolet. Applied Physics B: Lasers and Optics, 96(4):757-761, 2009.

[94] Jens Möhring, Tiago Buckup, C. Stefan Lehmann, and Marcus Motzkus. Generation of phasecontrolled ultraviolet pulses and characterization by a simple autocorrelator setup. J. Opt. Soc. Am. B, 26(8):1538-1544, 2009.

[95] Joshua C. Vaughan, Thomas Hornung, T. Feurer, and Keith A. Nelson. Diffraction-based femtosecond pulse shaping with a two-dimensional spatial light modulator. Optics Letters, 30(3):323-325, 2005.

$[96]$ * E. Frumker and Y. Silberberg. Phase and amplitude pulse shaping with two-dimensional phaseonly spatial light modulators. J. Opt. Soc. Am. B, 24(12):2940, 2007.

[97] Takashi Tanigawa, Yu Sakakibara, Shaobo Fang, Taro Sekikawa, and Mikio Yamashita. Spatial light modulator of 648 pixels with liquid crystal transparent from ultraviolet to near-infrared and its chirp compensation application. Opt. Lett., 34(11):1696-1698, 2009.

[98] Howe Siang Tan, W. S. Warren, and E. Schreiber. Generation and amplification of ultrashort shaped pulses in the visible by a two-stage noncollinear optical parametric process. Opt. Lett., 26(22):1812, 2001.

[99] M. E. Fermann, V. da Silva, D. A. Smith, Y. Silberberg, and A. M. Weiner. Shaping of ultrashort optical pulses by using an integrated acousto-optic tunable filter. Opt. Lett., 18(18):1505-1507, 
1993.

[100] **F. Verluise, V. Laude, Z. Cheng, C. Spielmann, and P. Tournois. Amplitude and phase control of ultrashort pulses by use of an acousto-optic programmable dispersive filter: pulse compression and shaping. Opt. Lett., 25(8):575, 2000.

[101] *Sebastien Coudreau, Daniel Kaplan, and Pierre Tournois. Ultraviolet acousto-optic programmable dispersive filter laser pulse shaping in kdp. Opt. Lett., 31(12):1899, 2006.

$[102]{ }^{* *}$ D. Kaplan and P. Tournois. Theory and performance of the acousto optic programmable dispersive filter used for femtosecond laser pulse shaping. Journal de Physique IV, 12:Pr5-69, 2002 .

[103] Jzsef Seres, Alexander Müller, Enikö Seres, Kevin O'Keeffe, Miklós Lenner, Richard F. Herzog, Daniel Kaplan, Christian Spielmann, and Ferenc Krausz. Sub-10-fs, terawatt-scale ti:sapphire laser system. Optics Letters, 28(19):1832-1834, 2003.

[104] Antoine Monmayrant, Arnaud Arbouet, Bertrand Girard, Béatrice Chatel, Benjamin J. Whitaker, A. Barman, and Daniel Kaplan. Optimisation of nopa output pulse shaping using an aopdf with dispersion self-correction. Appl. Phys. B, 81(2-3):177, 2005.

[105] D. H. Reitze, S. Kazamias, F. Weihe, G. Mullot, D. Douillet, F. Auge, O. Albert, V. Rarnanathan, L. P. Chambaret, D. Hulin, and P. Balcou. Enhancement of high-order harmonic generation at tuned wavelengths through adaptive control. Optics Letters, 29(1):86-8, 2004.

[106] D. N. Papadopoulos, M. Hanna, F. Druon, and P. Georges. Active spectral phase control by use of an acousto-optic programmable filter in high-repetition rate sub- 80 fs nonlinear fiber amplifiers. Optics letters, 33:1431, 2008.

[107] N.T. Form, J.B.C. Whitaker, and C. Meier. Enhancing the probablility of three-photon absorption in iodine through pulse shaping. J. Phys. B, 41(7), 2008.

[108] S. Weber, M. Barthelemy, and B. Chatel. Direct shaping of tunable uv ultrashort pulses. Applied Physics B: Lasers and Optics, 98:323-326, 2009.

[109] P. Tournois. Design of acousto-optic programmable filters in mercury halides for mid-infrared laser pulse shaping. Optics Communication, 281:4054, 2008.

[110] Nicholas T. Form, Robert Burbidge, Jan Ramon, and Benjamin J. Whitaker. Parameterization of an acousto-optic programmable dispersive filter for closed-loop learning experiments. Journal of Modern Optics, 55(1):197 - 209.

[111] N. Belabas, J. P. Likforman, L. Canioni, B. Bousquet, and M. Joffre. Coherent broadband pulse shaping in the mid infrared. Optics Letters, 26(10):743-5, 2001.

[112] Robert A. Kaindl, Matthias Wurm, Klaus Reimann, Peter Hamm, Andrew M. Weiner, and Michael Woerner. Generation, shaping, and characterization of intense femtosecond pulses tunable from 3 to $20 \mu \mathrm{m}$. J. Opt. Soc. Am. B, 17(12):2086-2094, 2000.

[113] M. Hacker, T. Feurer, R. Sauerbrey, T. Lucza, and G. Szabo. Programmable femtosecond laser pulses in the ultraviolet. Journal of the Optical Society of America B (Optical Physics), 18(6):866-71, 2001.

$[114]{ }^{* *}$ C. Schriever, S. Lochbrunner, M. Optiz, and E. Riedle. 19 fs shaped ultraviolet pulses. Opt. Lett., 31(4):543, 2006.

[115] B. Broers, H. B. V. Vandenheuvell, and L. D. Noordam. Large interference effects of small chirp observed in 2-photon absorption. Opt. Commun., 91(1-2):57, 1992.

[116] F. O. Koller, K. Haiser, M. Huber, T.E. Schrader, N. Regner, W.J. Schreier, and W. Zinth. Generation of narrowband subpicosecond midinfrared pulses via difference frequency mixing of chirped near-infrared pulses. Opt. Lett., 32(22):3339, 2007.

[117] F. Raoult, A. C. L. Boscheron, D. Husson, C. Sauteret, A. Modena, V. Malka, F. Dorchies, and A. Migus. Efficient generation of narrow-bandwidth picosecond pulses by frequency doubling of femtosecond chirped pulses. Opt. Lett., 23(14):1117, 1998.

[118] D. Zeidler, T. Witte, D. Proch, and M. Motzkus. Optical parametric amplification of a shaped white-light continuum. Optics Letters, 26(23):1921-3, 2001.

[119] M. M. Wefers and K. A. Nelson. Programmable phase and amplitude femtosecond pulse shaping. 
Opt. Lett., 18(23):2032, 1993.

[120] A. Präkelt, M. Wollenhaupt, A. Assion, C. Horn, C. Sarpe-Tudoran, M. Winter, and T. Baumert. Compact, robust and flexible setup for femtosecond pulse shaping. Rev. Sci. Instr., 74(11):4950, 2003.

[121] G. Stobrawa, M. Hacker, T. Feurer, D. Zeidler, M. Motzkus, and F. Reichel. A new high-resolution femtosecond pulse shaper. Applied Physics B, 72(5):627-30, 2001.

[122] T. Binhammer, E. Rittweger, R. Ell, F.X. Kartner, and U. Morgner. Prism-based pulse shaper for octave spanning spectra. Quantum Electronics, IEEE Journal of, 41(12):1552-1557, 2005.

[123] L. Xu, N. Nakagawa, R. Morita, H. Shigekawa, and M. Yamashita. Programmable chirp compensation for 6-fs pulse generation with a prism-pair-formed pulse shaper. Quantum Electronics, IEEE Journal of, 36(8):893-899, 2000.

[124] Reimer Selle, Patrick Nuernberger, Florian Langhojer, Frank Dimler, Susanne Fechner, Gustav Gerber, and Tobias Brixner. Generation of polarization-shaped ultraviolet femtosecond pulses. Opt. Lett., 33(8):803-805, 2008.

[125] T. Witte, D. Zeidler, D. Proch, K. L. Kompa, and M. Motzkus. Programmable amplitude- and phase-modulated femtosecond laser pulses in the mid-infrared. Opt. Lett., 27(2):131-133, 2002.

[126] Howe-Siang Tan and Warren Warren. Mid infrared pulse shaping by optical parametric amplification and its application to optical free induction decay measurement. Opt. Express, 11(9):1021-1028, 2003.

[127] **John M. Dudley, Ian A. Walmsley, and Rick Trebino. Measurement of ultrashort electromagnetic pulses. J. Opt. Soc. Am. B, 25(6):MU1-MU2, 2008.

[128] K. Sala, G. Kenney-Wallace, and G. Hall. Cw autocorrelation measurements of picosecond laser pulses. Quantum Electronics, IEEE Journal of, 16(9):990-996, 1980.

[129] Jean-Claude M. Diels, Joel J. Fontaine, Ian C. McMichael, and Francesco Simoni. Control and measurement of ultrashort pulse shapes (in amplitude and phase) with femtosecond accuracy. Appl. Opt., 24(9):1270-1282, 1985.

[130] Antoine Monmayrant, Béatrice Chatel, and Bertrand Girard. Real time quantum state holography using coherent transients. Opt. Commun., 264(2):256, 2006.

[131] E. Goulielmakis, M. Uiberacker, R. Kienberger, A. Baltuska, V. Yakovlev, A. Scrinzi, Th. Westerwalbesloh, U. Kleineberg, U. Heinzmann, M. Drescher, and F. Krausz. Direct measurement of light waves. Science, 305(5688):1267-1269, 2004.

[132] C. Froehly, A. Lacourt, and J. C. Vienot. Notions de réponse impulsionnelle et de fonction de transfert temporelles des pupilles optiques. Journal d'optique, 4:183, 1973.

[133] Mitsuo Takeda, Hideki Ina, and Seiji Kobayashi. Fourier-transform method of fringe-pattern analysis for computer-based topography and interferometry. J. Opt. Soc. Am., 72(1):156-160, 1982.

[134] **L. Lepetit, G. Cheriaux, and M. Joffre. Linear techniques of phase measurement by femtosecond spectral interferometry for applications in spectroscopy. J. Opt. Soc. Am B, 12:2467, 1995.

[135] Christophe Dorrer and Manuel Joffre. Characterization of the spectral phase of ultrashort light pulses. Académie des sciences, 2(4):1415, 2001.

[136] ***D. J. Kane and R. Trebino. Characterization of arbitrary femtosecond pulses using frequencyresolved optical gating. I.E.E.E. Journal of Quantum Electronics, 29(2):571-579, 1993.

[137] Daniel J. Kane and Rick Trebino. Single-shot measurement of the intensity and phase of an arbitrary ultrashort pulse by using frequency-resolved optical gating. Opt. Lett., 18(10):823825, 1993.

[138] *D. J. Kane. Recent progress toward real-time measurement of ultrashort laser pulses. IEEE Journal of Quantum Electronics, 35(4):421, 1999.

[139] Jerome Paye, Malini Ramaswamy, James G. Fujimoto, and Erich P. Ippen. Measurement of the amplitude and phase of ultrashort light pulses from spectrally resolved autocorrelation. Opt. Lett., 18(22):1946, 1993.

[140] P. O'Shea, M. Kimmel, X. Gu, and R. Trebino. Highly simplified device for ultrashort-pulse 
measurement. Optics Letters, 26(12):932-934, 2001.

[141] D. T. Reid, P. Loza-Alvarez, C. T. A. Brown, T. Beddard, and W. Sibbett. Amplitude and phase measurement of mid-infrared femtosecond pulses by using cross-correlation frequency-resolved optical gating. Opt. Lett., 25(19):1478-1480, 2000.

[142] S. Linden, J. Kuhl, and H. Giessen. Amplitude and phase characterization of weak blue ultrashort pulses by downconversion. Optics Letters, 24:569, 1999.

[143] Masaaki Tsubouchi and Takamasa Momose. Cross-correlation frequency-resolved optical gating for mid-infrared femtosecond laser pulses by an aggages4 crystal. Opt. Lett., 34(16):2447-2449, 2009.

[144] John Dudley, Xun Gu, Lin Xu, Mark Kimmel, Erik Zeek, Patrick O'Shea, Rick Trebino, Stephane Coen, and Robert Windeler. Cross-correlation frequency resolved optical gating analysis of broadband continuum generation in photonic crystal fiber: simulations and experiments. Opt. Express, 10(21):1215-1221, 2002.

[145] Thomas Y. F. Tsang, Marco A. Krumbugel, Kenneth W. DeLong, David N. Fittinghoff, and R. Trebino. Frequency-resolved optical-gating measurements of ultrashort pulses using surface third-harmonic generation. Optics Letters, 21(17):1381-1383, 1996.

[146] Y. Mairesse and F. Quere. Frequency-resolved optical gating for complete reconstruction of attosecond bursts. Phys. Rev. A, 71(1):011401, 2005.

[147] K. W. DeLong, Rick Trebino, J. Hunter, and W. E. White. Frequency-resolved optical gating with the use of second-harmonic generation. J. Opt. Soc. Am. B, 11(11):2206-2215, 1994.

[148] Selcuk Akturk, Mark Kimmel, Rick Trebino, Sergey Naumov, Evgeni Sorokin, and Irina Sorokina. Measuring several-cycle 1.5-m pulses using frequency-resolved optical gating. Opt. Express, 11(25):3461-3466, 2003.

[149] B. A. Richman, M. A. Krumbügel, and R. Trebino. Temporal characterization of mid-ir freeelectron-laser pulses by frequency-resolved optical gating. Opt. Lett., 22(10):721-723, 1997.

[150] Gero Stibenz and Günter Steinmeyer. Interferometric frequency-resolved optical gating. Opt. Express, 13(7):2617-2626, 2005.

[151] John N. Sweetser, David N. Fittinghoff, and Rick Trebino. Transient-grating frequency-resolved optical gating. Opt. Lett., 22(8):519-521, 1997.

[152] Dongjoo Lee, Selcuk Akturk, Pablo Gabolde, and Rick Trebino. Experimentally simple, extremely broadband transient-grating frequency-resolved-opticalgating arrangement. Opt. Express, 15(2):760-766, 2007.

[153] C. Iaconis and I. A. Walmsley. Spectral phase interferometry for direct electric-field reconstruction of ultrashort optical pulses. Optics Letters, 23(10):792-794, 1998.

[154] ***M.E. Anderson, A. Monmayrant, S.-P. Gorza, P. Wasylczyk, and I.A. Walmsley. Spider: A decade of measuring ultrashort pulses. Laser Physics Letters, 5(4):259-266, 2008.

[155] M. Hirasawa, N. Nakagawa, K. Yamamoto, R. Morita, H. Shigekawa, and M. Yamashita. Sensitivity improvement of spectral phase interferometry for direct electric-field reconstruction for the characterization of low-intensity femtosecond pulses. Applied Physics B: Lasers and Optics, 74(0):s225, 2002.

[156] G. Stibenz and G. Steinmeyer. High dynamic range characterization of ultrabroadband whitelight continuum pulses. Optics Express, 12(25):6319-6325, 2004.

[157] Christophe Dorrer, P. Londero, and I. A. Walmsley. Homodyne detection in spectral phase interferometry for direct electric field reconstruction. Optics Letters, 26(19):1510, 2001.

[158] Peter Baum, Stefan Lochbrunner, and Eberhard Riedle. Zero-additional-phase spider: full characterization of visible and sub-20-fs ultraviolet pulses. Optics Letters, 29(2):210-212, 2004.

[159] **Peter Baum and Eberhard Riedle. Design and calibration of zero-additional-phase spider. $J$. Opt. Soc. Am. B, 22(9):1875-1883, 2005.

[160] A. Monmayrant, M. Joffre, T. Oksenhendler, R. Herzog, D. Kaplan, and P. Tournois. Timedomain interferometry for direct electric-field reconstruction by use of an acousto-optic programmable filter and a two-photon detector. Optics Letters, 28(4):278, 2003. 
[161] C. Ventalon, J. M. Fraser, and M. Joffre. Time-domain interferometry for direct electric field reconstruction of mid-infrared femtosecond pulses. Optics Letters, 28(19):1826-8, 2003.

[162] E. M. Kosik, A. S. Radunsky, I. A. Walmsley, and C. Dorrer. Interferometric technique for measuring broadband ultrashort pulses at the sampling limit. Optics Letters, 30(3):326-328, 2005.

[163] S. P. Gorza, P. Wasylczyk, and I. A. Walmsley. Spectral shearing interferometry with spatially chirped replicas for measuring ultrashort pulses. Opt. Express, 15(23):15168-15174, 2007.

[164] J. Bethge, C. Grebing, and G. Steinmeyer. A fast gabor wavelet transform for high-precision phase retrieval in spectralinterferometry. Opt. Express, 15(22):14313-14321, 2007.

[165] Jens Bethge and Günter Steinmeyer. Numerical fringe pattern demodulation strategies in interferometry. Review of Scientific Instruments, 79(7):073102, 2008.

[166] L. Gallmann, D. H. Sutter, N. Matuschek, G. Steinmeyer, U. Keller, C. Iaconis, and I. A. Walmsley. Characterization of sub-6-fs optical pulses with spectral phase interferometryfor direct electric-field reconstruction. Optics Letters, 24(18):1314-1316, September 1999.

[167] C. Dorrer. Implementation of spectral phase interferometry for direct electric-field reconstruction with a simultaneously recorded reference interferogram. Optics Letters, 24(21):1532-4, 1999.

[168] Gero Stibenz and Günter Steinmeyer. Optimizing spectral phase interferometry for direct electricfield reconstruction. Review of Scientific Instruments, 77(7):073105, 2006.

[169] P. Londero, M. E. Anderson, C. Radzewicz, C. Iaconis, and I. A. Walmsley. Measuring ultrafast pulses in the near-ultraviolet using spectral phase interferometry for direct electric field reconstruction. Journal of Modern Optics, 50(2):179-184, February 2003.

[170] C. Dorrer and I. Kang. Highly sensitive direct characterization of femtosecond pulses by electrooptic spectral shearing interferometry. Optics Letters, 28(6):477-479, March 2003.

[171] Inuk Kang, Christophe Dorrer, and Francesco Quochi. Implementation of electro-optic spectral shearing interferometry for ultrashort pulse characterization. Optics Letters, 28(22):2264-2266, 2003.

[172] Kevin J. Kubarych, Manuel Joffre, Amy Moore, Nadia Belabas, and David M. Jonas. Mid-infrared electric field characterization using a visible charge-coupled-device-based spectrometer. Opt. Lett., 30(10):1228-1230, 2005.

[173] Y. Mairesse, O. Gobert, P. Breger, H. Merdji, P. Meynadier, P. Monchicourt, M. Perdrix, P. Salieres, and B. Carre. High harmonic xuv spectral phase interferometry for direct electricfield reconstruction. Phys. Rev. Lett., 94(17):173903, 2005.

[174] *Adam S Wyatt, Ian A Walmsley, Gero Stibenz, and Günter Steinmeyer. Sub-10 fs pulse characterization using spatially encoded arrangement for spectral phase interferometry for direct electric field reconstruction. Opt Lett, 31(12):1914-1916, 2006.

[175] A.S. Radunsky, I.A. Walmsley, S.P. Gorza, and P. Wasylczyk. Compact spectral shearing interferometer for ultrashort pulse characterization. Optics Letters, 32(2):181-183, 2007.

[176] J. R. Birge, R. Ell, and F. X. Kärtner. Two-dimensional spectral shearing interferometry for few-cycle pulse characterization. Optics Letters, 31(13):2063-2065, 2006.

[177] J.-P. Foing, J.-P. Likforman, M. Joffre, and A. Migus. Femtosecond pulse phase measurement by spectrally resolved up-conversion: application to continuum compression. Quantum Electronics, IEEE Journal of, 28(10):2285-2290, 1992.

[178] A. Galler and T. Feurer. Pulse shaper assisted short laser pulse characterization. Applied Physics B: Lasers and Optics, 90(3):427-430, 2008.

[179] N. Forget, S. Coudreau, F. Lepetit, O. Albert, and T. Oksenhendler. Achromatic and singlebeam pulse characterization technique for visible-uv pulses based on direct uv pulse shaping and cross-polarized wave generation. In CLEO/Europe and IQEC 2007 Conference Digest, page CF4_5. Optical Society of America, 2007.

[180] N. Forget, M. Joffre, S. Coudreau, and T. Oksenhendler. Toward programmable ultrashort pulse characterization. In CLEO/Europe and IQEC 2007 Conference Digest, page CF_16. Optical Society of America, 2007. 
[181] Bernhard von Vacano, Tiago Buckup, and Marcus Motzkus. Shaper-assisted collinear spider: fast and simple broadband pulse compression in nonlinear microscopy. Journal of the Optical Society of America B (Optical Physics), 24(5):1091-1100, 2007.

[182] Yves Coello, Vadim V. Lozovoy, Tissa C. Gunaratne, Bingwei Xu, Ian Borukhovich, Chien hung Tseng, Thomas Weinacht, and Marcos Dantus. Interference without an interferometer: a different approach to measuring, compressing, and shaping ultrashort laser pulses. J. Opt. Soc. Am. B, 25(6):A140-A150, 2008.

[183] Bingwei Xu, Yves Coello, Vadim V. Lozovoy, D. Ahmasi Harris, and Marcos Dantus. Pulse shaping of octave spanning femtosecond laser pulses. Opt. Express, 14(22):10939-10944, 2006.

$[184]{ }^{* *}$ V. V. Lozovoy, I. Pastirk, and M. Dantus. Multiphoton intrapulse interference. iv. ultrashort laser pulse spectral phase characterization and compensation,. Opt. Lett., 29(7):775, 2004.

[185] Vadim V. Lozovoy, Bingwei Xu, Yves Coello, and Marcos Dantus. Direct measurement of spectral phase for ultrashort laser pulses. Opt. Express, 16(2):592-597, 2008. 


\title{
A newcomer's guide to ultrashort pulse shaping and characterization
}

\author{
Antoine Monmayrant ${ }^{1,2}$, Sébastien Weber $^{3}$, and Béatrice \\ Chatel $^{3}$ \\ ${ }^{1}$ CNRS-LAAS ; 7 avenue du colonel Roche, F-31077 Toulouse, France \\ ${ }^{2}$ Université de Toulouse ; UPS, INSA, INP, ISAE ; LAAS, F-31077 Toulouse, France \\ ${ }^{3}$ CNRS-Université de Toulouse,UPS, Laboratoire Collisions, Agrégats Réactivité, \\ IRSAMC,F-31062 Toulouse, France. \\ E-mail: beatrice.chatel@irsamc.ups-tlse.fr
}

\begin{abstract}
This tutorial will overview the most widespread pulse shaping and characterization techniques of ultrashort pulses. It will also point their range of usage and some of their limitations.
\end{abstract}

PACS numbers: 42.65.Re; 42.65.Ky; 42.30.Kq

Submitted to: J. Phys. B: At. Mol. Phys.

\section{Introduction}

2. Definition of the electric field and of the Wigner function

2.1. Definition of the electric field
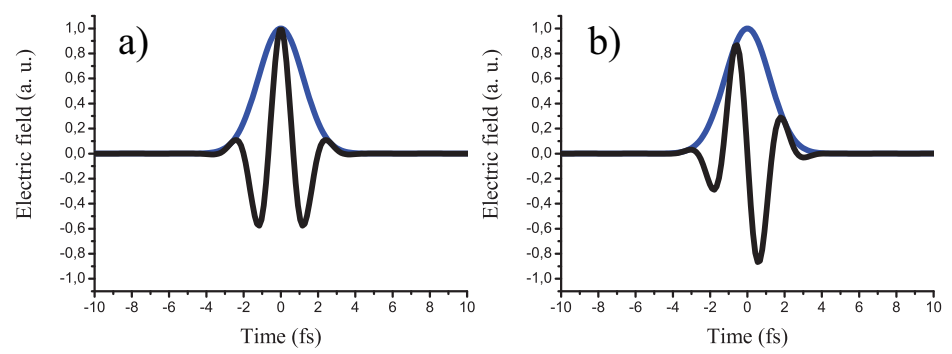

Figure 1. $\varepsilon(t)$ (in black) and envelope (in blue) of the electric field of a short laser pulse in two case of carrier envelope phase. The pulses has a central wavelength of $800 \mathrm{~nm}$ and a FWHM of $2 \mathrm{fs}$. In a) a 0 radian CEP shows a cosine pulse and in b) a $\pi / 2$ radian CEP shows a sine pulse.

\subsection{How to represent a short pulse?}




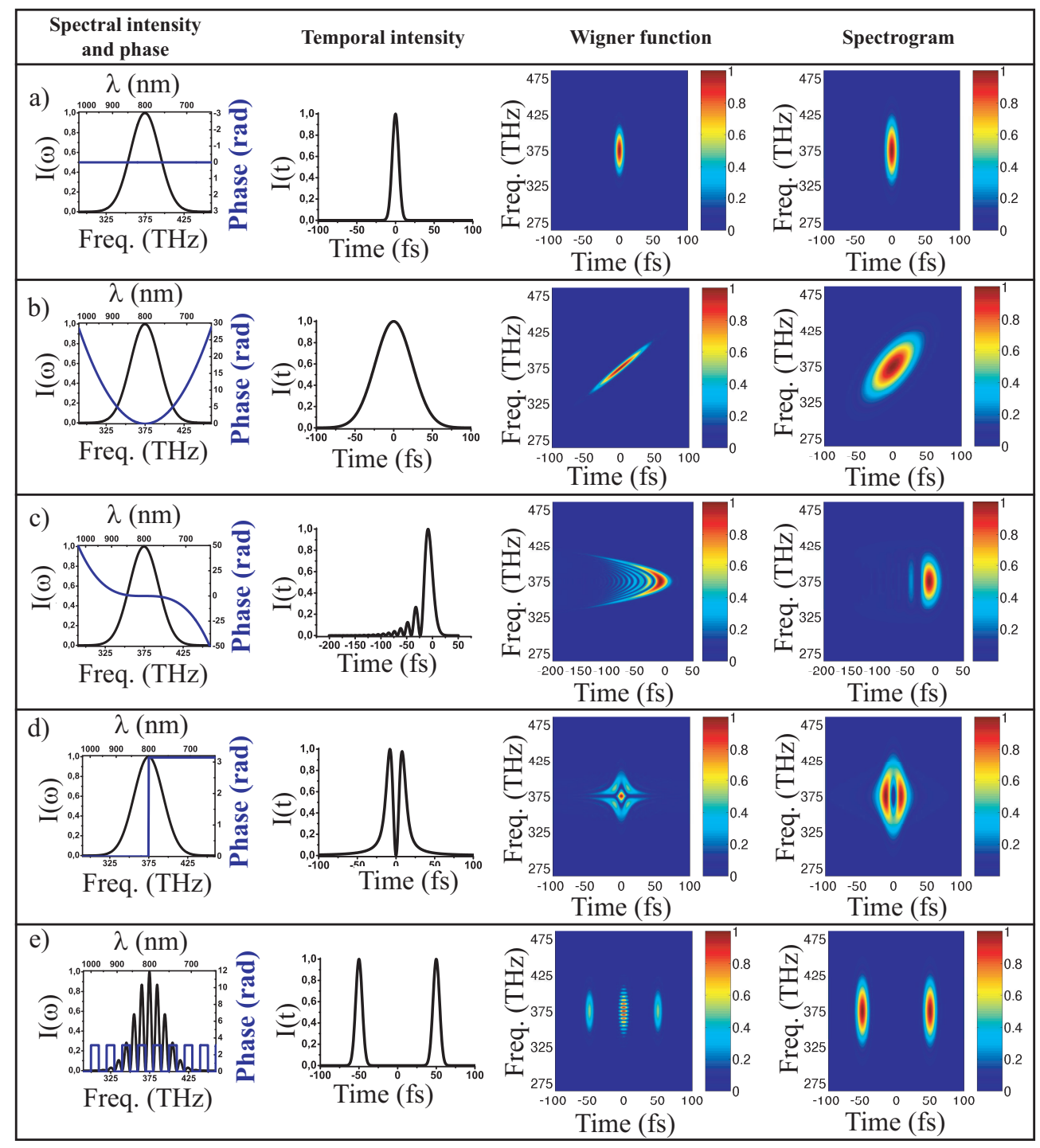

Figure 2. Pulse shaping gallery; (a) Fourier transform pulse, (b) chirp pulse, (c) cubic phase,(d) spectral $\pi$-jump,(e) two-pulse sequence.

\section{Arbitrary Pulse shaping of ultrashort pulses}

\subsection{Generalities}

3.2. A spatial mask in the Fourier plane of a zero dispersion line 3.2.1. Introduction

3.2.2. Shaping theory in few equations 
a) Frequency domain

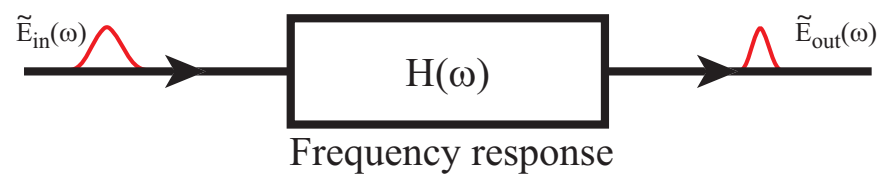

b) Time domain

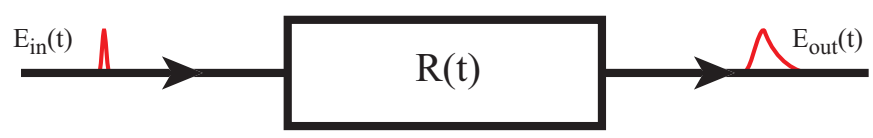

Impulse response

Figure 3. Pulse shaping by linear filtering. a) Time domain.b) Frequency domain.

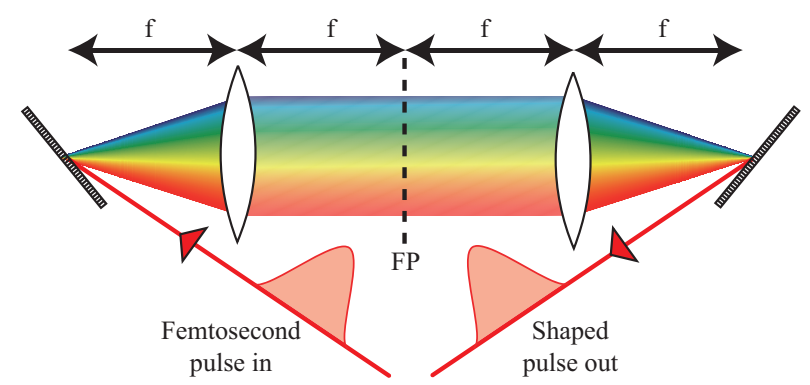

Figure 4. A zero dispersion line composed of two gratings and two lenses of focal length $\mathrm{f}$, arranged in a $4 \mathrm{f}$ setup. The output pulse is identical to the input pulse. In the Fourier plane FP, all the spectral components are spatially separated and focused.

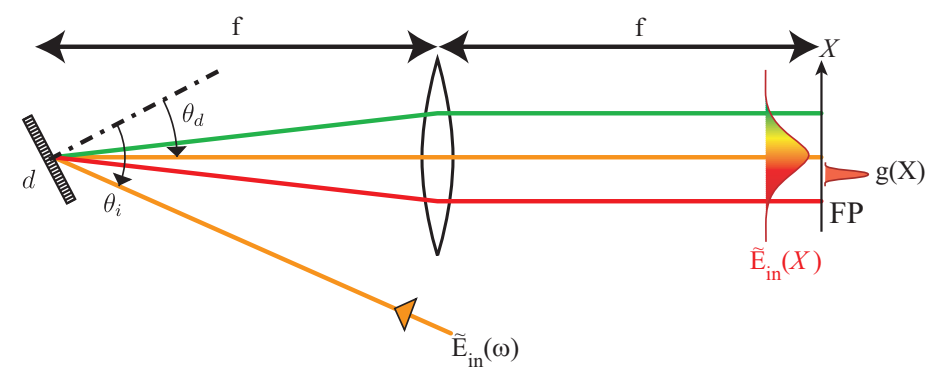

Figure 5. Half a $4 \mathrm{f}$ line: The Fourier plane is situated in the back focal plane of the lens. $f$ is the focal length. $X$ is the spatial coordinate in this plane. $g(X)$ is the spatial extension of a given frequency component. $\theta_{i}$ is the incident angle on the grating. $d$ is the grating period. $\theta_{d}$ the diffraction angle.

\subsubsection{Spatio-temporal coupling}

\subsection{The most common Masks}

\subsubsection{The liquid crystal mask}



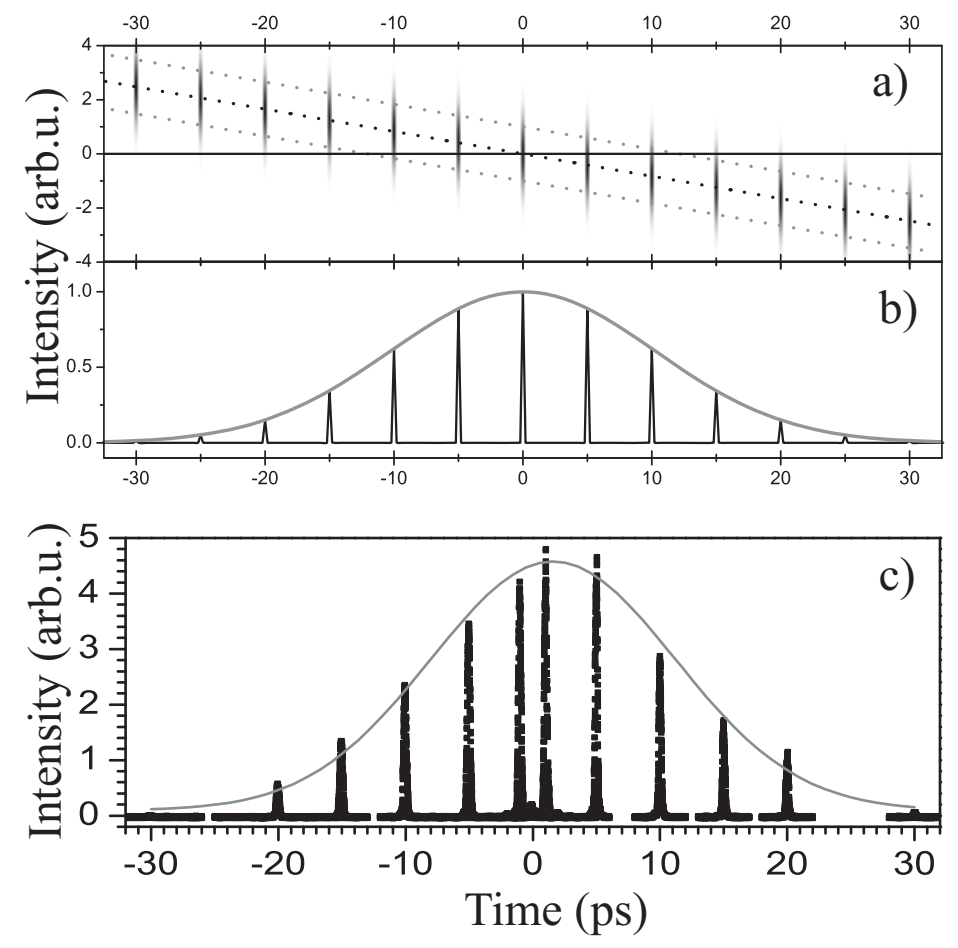

Figure 6. Space-time coupling in the case of a delay: a) Theoretical plot versus time and transverse position of a shaped pulse for various programmed delays. b) Cross-section of plot (a) along the axis of propagation (transverse position $=0$ ). c) Experimental cross-correlations of shaped pulses for various delays.

a)

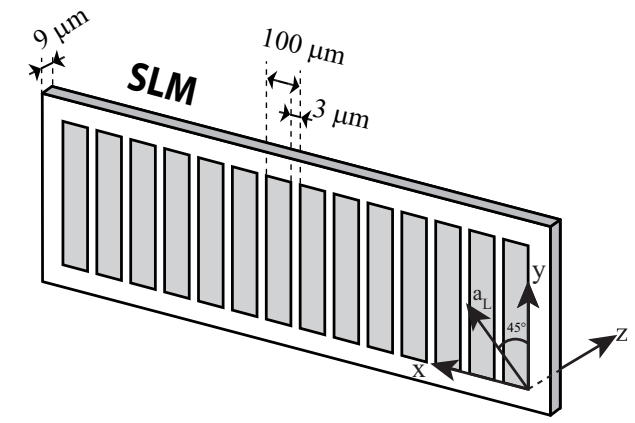

b)

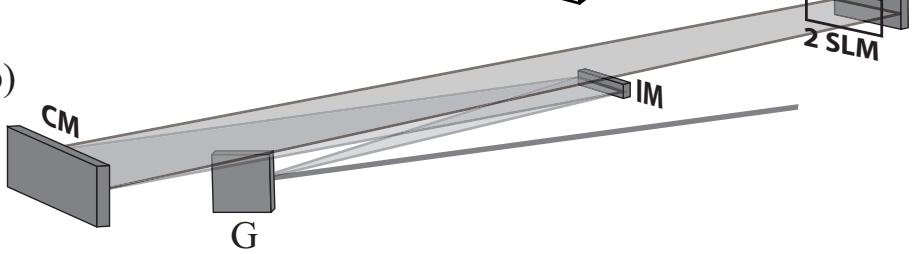

Figure 7. a) 640 pixels Liquid Crystals spatial light modulator. $a_{L}$ is the anchorage direction and represents the slow axis of the crystal. b) A folded zero dispersion line geometry with almost no optical aberration. G : grating; IM : intermediate mirror; CM: cylindrical mirror; FM: folding mirror; SLM : liquid crystal mask. 


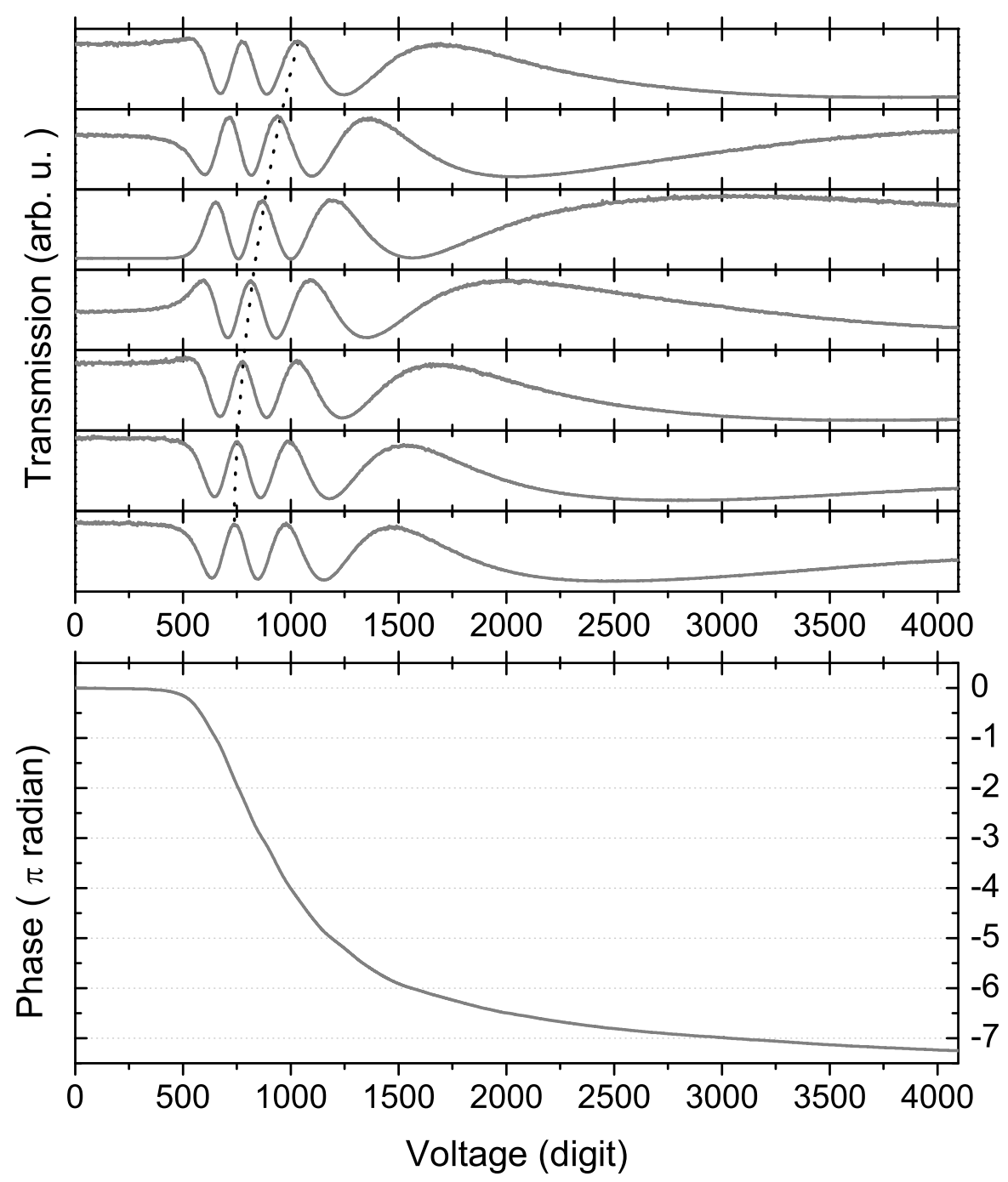

Figure 8. Phase-voltage calibration at $795 \mathrm{~nm}$ of a CRI128 LC-SLM: Top curves: transmitted intensity as a function of the applied voltage, for several orientation of the waveplate; the position of the extrema depends on the extra phase added by the waveplate (dashed line is a guide to the eye). Bottom curve: Reconstructed phase as a function of the voltage.

RESULTS

LIMITATIONS

3.3.2. Acousto-optical modulator masks

ALIGNMENT AND CALIBRATION 


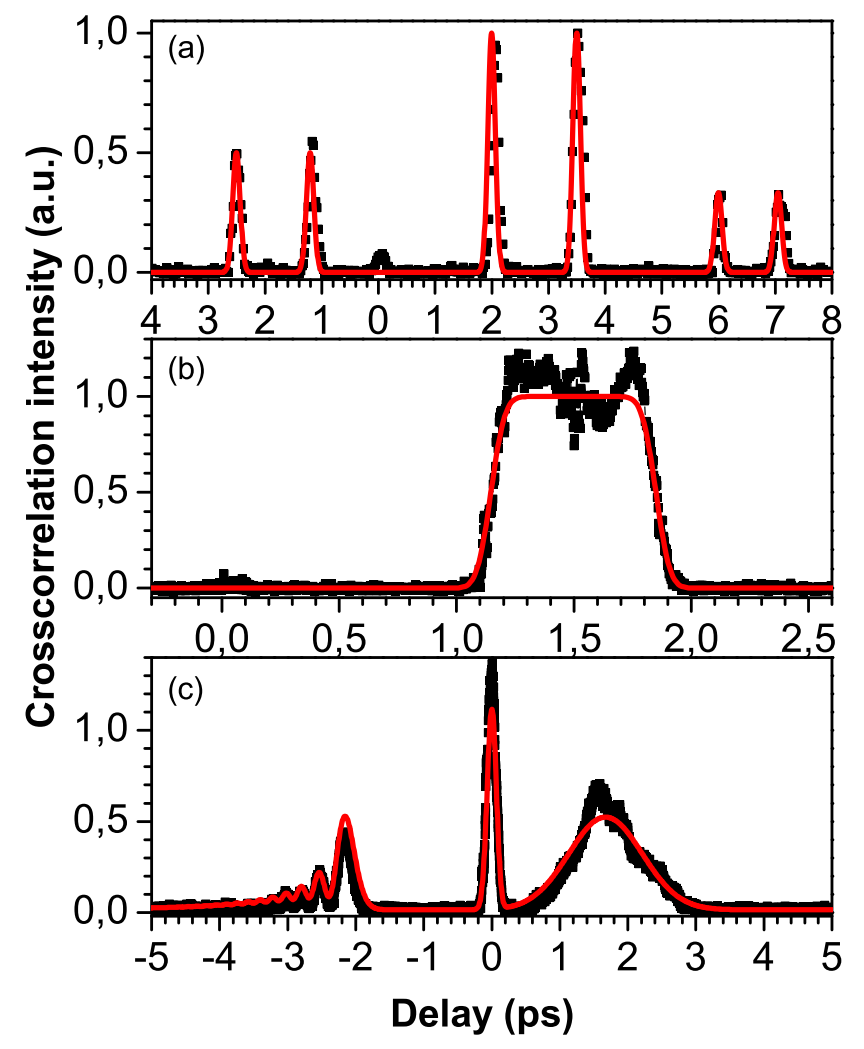

Figure 9. Three experimental waveforms are presented: (a) three equal amplitude pulses pairs with relative intensity 2:3:1, (b) a square pulse of $700 \mathrm{fs}$, and (c) three pulses with quadratic, no phase, and cubic phase.

\section{RESULTS}

\section{LIMITATIONS}

\subsubsection{Comparison between $L C$ and $A O M$ spatial light modulators}

\subsubsection{Other masks}

3.4. Shaping by phase transfer

3.4.1. The Acousto-Optic programmable Dispersive Filter (AOPDF)

ALIGNMENT AND CALIBRATION

RESULTS 

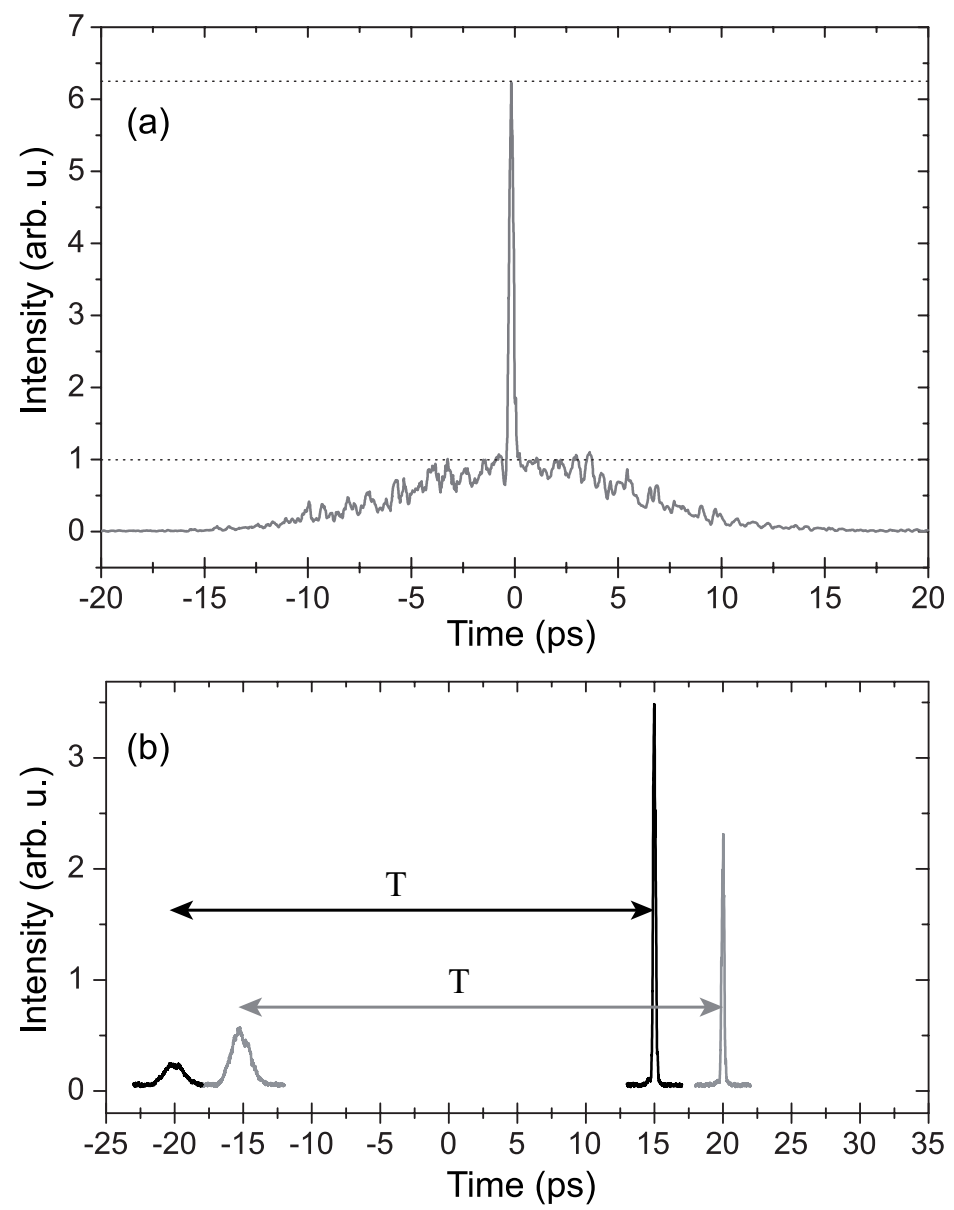

Figure 10. (a) Cross correlation of a chirped pulse with a reference ultrashort pulse. The gap-replica is clearly spotted. (b) Programmation of two delays : +15 ps ( black solid line) and +20 ps (gray solid line); Replica due to the pixellisation of the mask are clearly seen respectively around $-20 \mathrm{ps}$ and $-15 \mathrm{ps}$. Their temporal broadening is due to nonlinear dispersion in the Fourier plane.

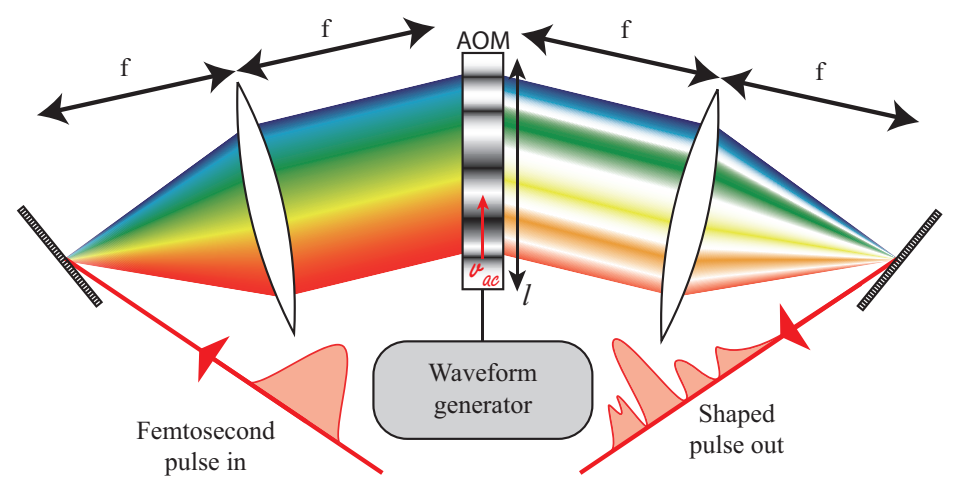

Figure 11. Programmable pulse shaping apparatus based on the use of a 4f-line and an Acousto-Optic Modulator. A radio frequency wave is generated and converted to an acoustic wave by a piezoelectric modulator. This traveling wave acts as a diffractive grating mask. 


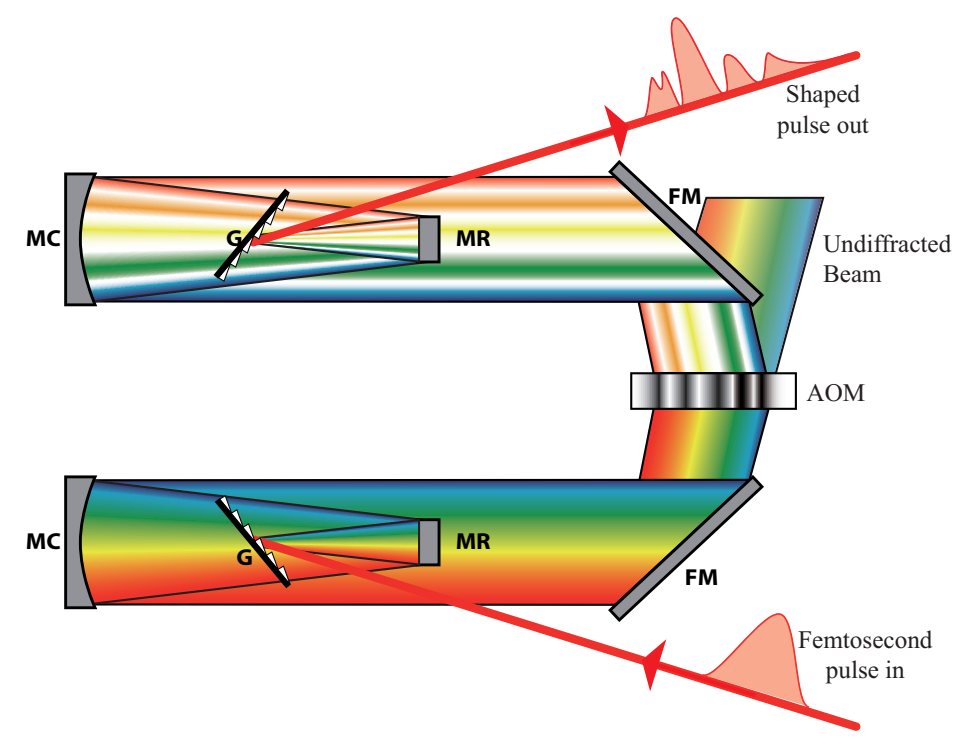

Figure 12. Experimental setup of a $4 \mathrm{f}$ line with an AOM spatial mask. The alignment of the line is made without the AOM. The alignment with the AOM is then made by the use of the two folding mirror placed at each side of the AOM. The first one is used to enter the AOM at Bragg angle whereas the second is used to compensate the diffraction angle. The length mismatch between the configuration with and without $\mathrm{AOM}$ is reduced by placing the two mirrors close to the AOM.

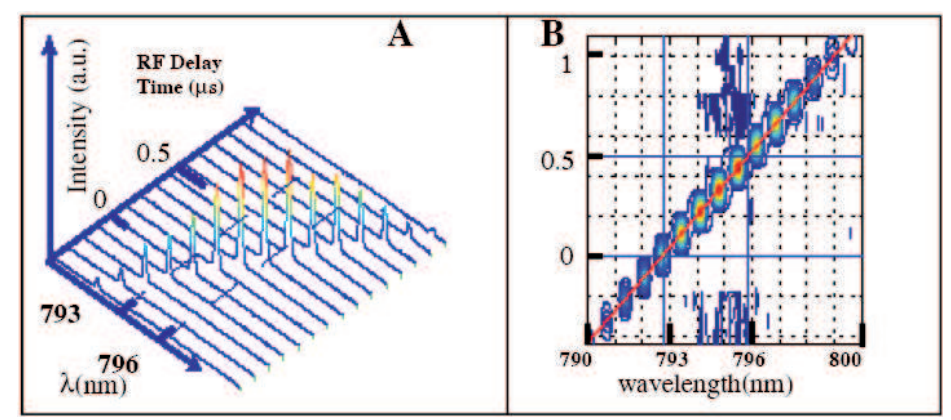

Figure 13. Figure from ref [1]. Calibration curves for AOM pulse shaper. A: each line represents a shaped spectrum created with a short RF pulse delayed in time in the AOM. Each delay corresponds to a different spatial position on the AOM leading to a different spectral position. This figure is used to calibrate the pulse shaper response function with respect to amplitude and time. B: data from A viewed as a contour plot. The solid red line fits the data, the coordinates are then used to calibrate the RF time with respect to optical wavelength.

\section{LIMITATIONS}

\subsubsection{Non linear frequency mixing}

\subsection{Conclusion}

\subsection{1. the complexity}




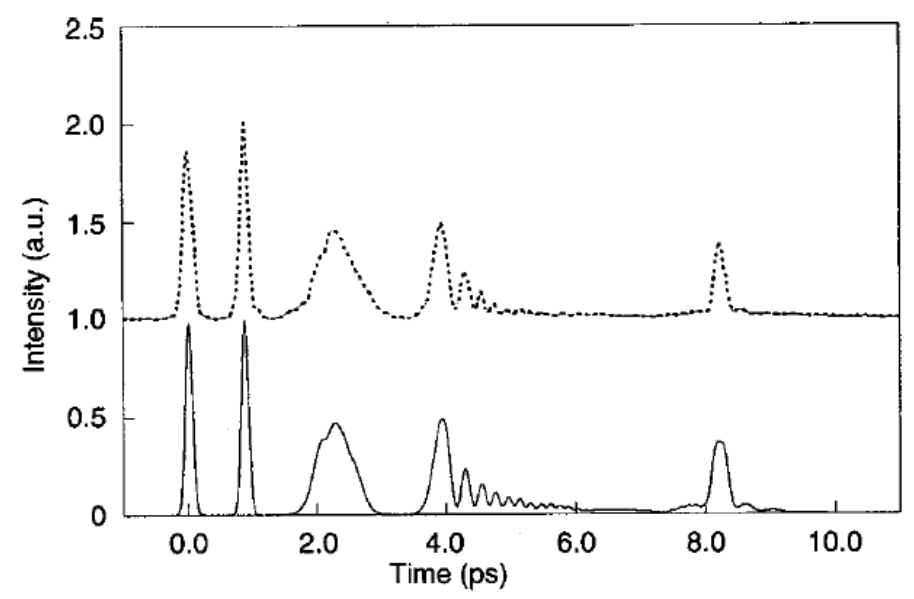

Figure 14. Figure from ref [2]. Dashed line : intensity cross-correlation trace of a shaped femtosecond pulse usinf AOM pulse shaper. The waveform used consists of an initial Fourier-Limited pulse followed in time by pulses shaped with linear, quadratic, cubic and quartic spectral phase. Solid line : theoretical trace.

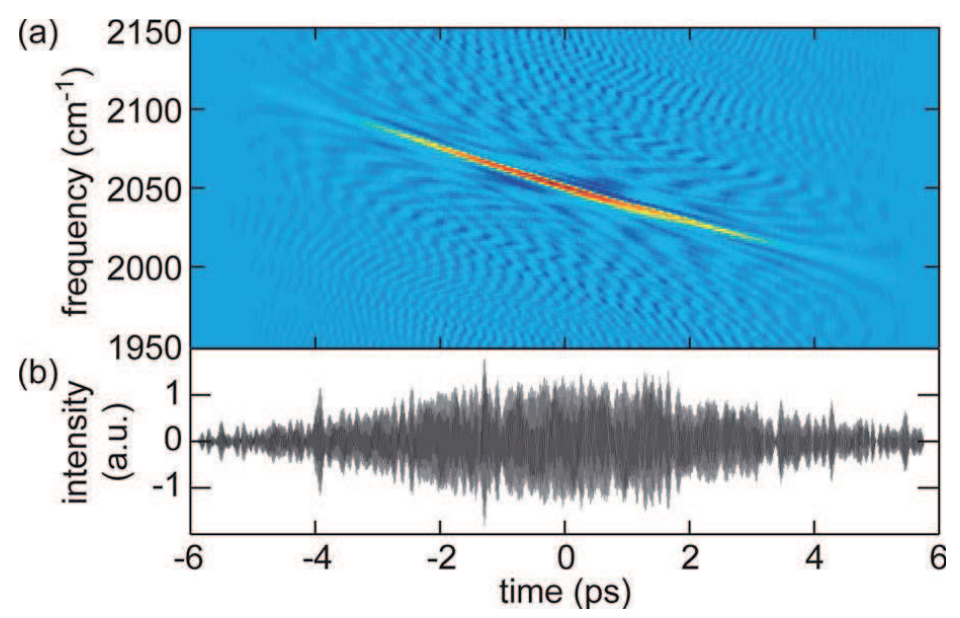

Figure 15. Figure from ref[3]. a) Wigner diagram obtained from (b). b) Cross correlation of a linearly chirped shaped pulse at 4.87 microns or $2050 \mathrm{~cm}^{-1}$ in the mid IR.

\subsubsection{Summary of the possibilities}




\begin{tabular}{|c|c|c|c|c|}
\hline SLM & LC & AOM & MEMM & LMDM (peu de ref!!) \\
\hline Control & $\begin{array}{c}\text { Amplitude } \\
\text { Phase }\end{array}$ & $\begin{array}{c}\text { Amplitude } \\
\text { Phase }\end{array}$ & Phase & Phase \\
\hline Parameter number & $128-640$ & $100-900$ & $240 \times 200$ & 19 (thèse Antoine) \\
\hline Refreshing rate & $10 \mathrm{~Hz}$ & $100 \mathrm{kHz}$ & $100 \mathrm{~Hz}$ & $5 \mathrm{kHz}$ (thèse Antoine) \\
\hline Laser repetition rate & $>80 \mathrm{MHz}$ & $<100 \mathrm{kHz}$ & $>80 \mathrm{MHz}$ & $>80 \mathrm{MHz}$ \\
\hline Efficiency & $60 \%$ & $10 \%$ to $30 \%$ & $\sim 85 \%$ & $\sim 80 \%$ \\
\hline Wavelength range $(\mathrm{nm})$ & $430-1600$ & $266-18000$ & $266-900$ & $500-1000,350,266$ \\
\hline
\end{tabular}

Table 1. Summary of available Spatial Light Modulators for 4f-line based pulse shapers. LC : liquid crystal, AOM : Acousto-Optic Modulator, MEMM : MicroElectro Mechanical Mirrors and LMDM : Linear Membrane Deformable Mirror.

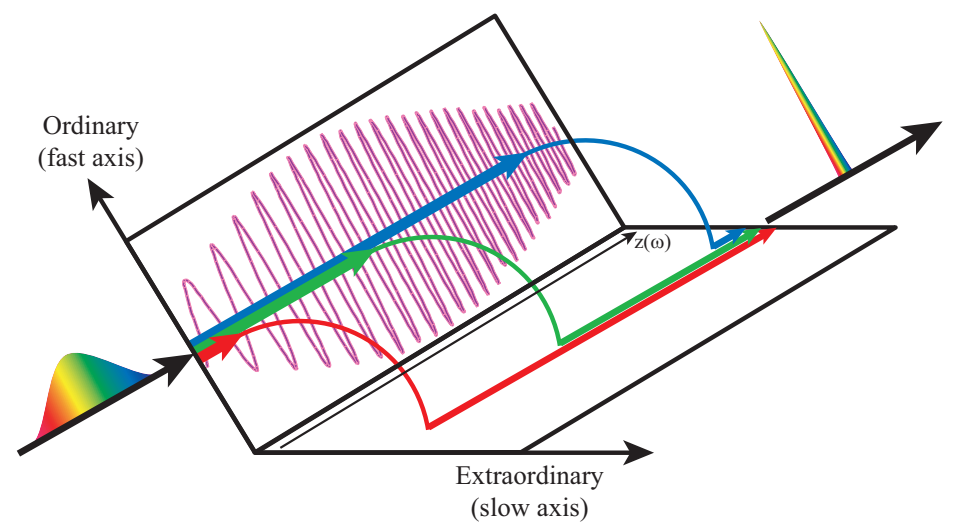

Figure 16. AOPDF principle: By Bragg diffraction acoustically assisted, the different spectral components can be switched along the extraordinary axis at different position in the crystal.

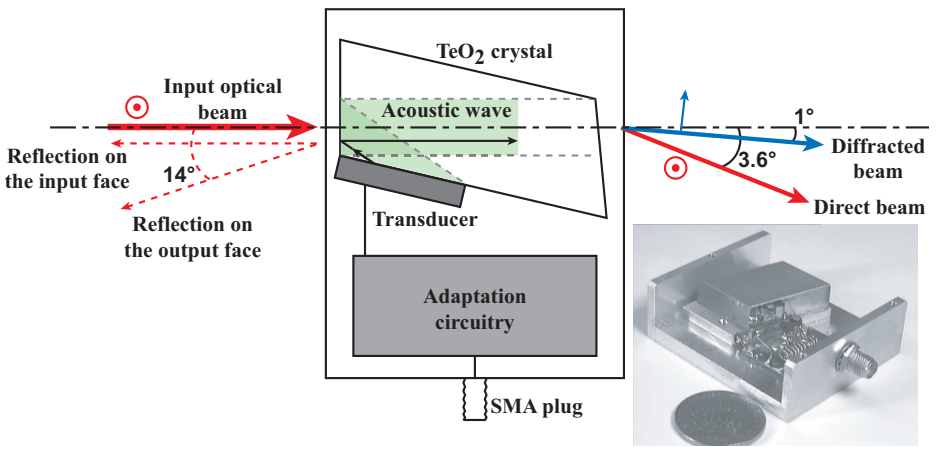

Figure 17. Experimental view of the Dazzler. 

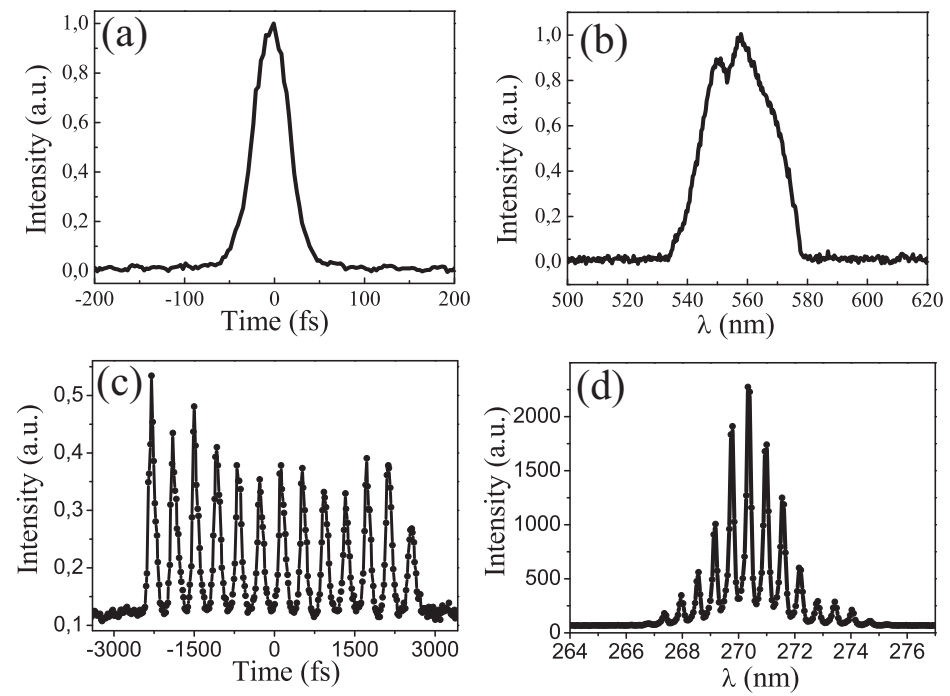

Figure 18. Results obtained with an AOPDF in the visible range: (a) autocorrelation of the output of a NOPA compressed by the AOPDF and (b) corresponding spectrum. In the UV range $(266 \mathrm{~nm})$ : cross-correlation of a 13-pulse sequence with a reference ultrashort pulse at $800 \mathrm{~nm}$ (c) and corresponding spectrum (d).

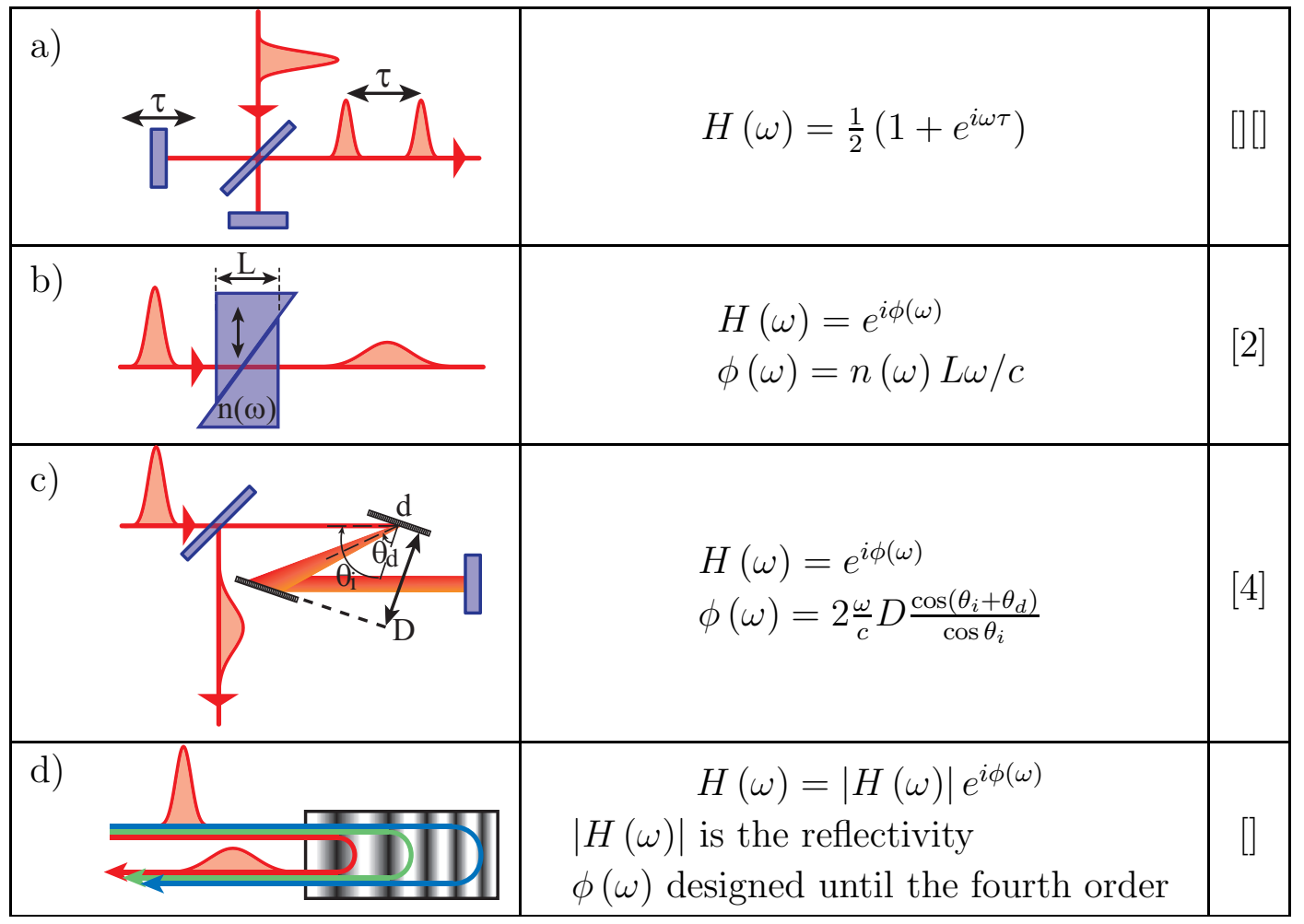

Table 2. Simple shaping setups. a) Michelson interferometer producing two delayed pulses, b) shaping by propagation through a length $\mathrm{L}$ of a dispersive bulk medium, c) grating compressor shaping and d) chirped mirror shaping. 


\begin{tabular}{|c|c|c|c|c|}
\hline & Mid IR & IR & Visible & UV \\
\hline LC- in a 4f line & & {$[5],[6],[7],[8],[9],[10]$} & {$[11]$} & \\
\hline AOM in a 4f line & {$[3]$} & {$[12]$} & {$[13]$} & {$[14],[15]$} \\
\hline $\begin{array}{c}\text { Deformable mirror } \\
\text { MEMs }\end{array}$ & & {$[16]$} & & {$[17]$} \\
\hline Phase -transfer & {$[18],[19],[20]$} & & & \\
\hline AOPDF & {$[22]$} & {$[23]$} & {$[24]$} & {$[25],[26]$} \\
\hline
\end{tabular}

Table 3. Summary of available pulse shapers in various spectral domains. 


\section{Characterization of ultrashort shaped pulses}

\subsection{Prerequisite and generality}

\subsection{Referenced complete characterization methods}

(a)

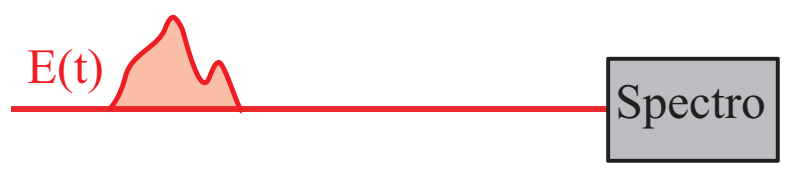

(b)

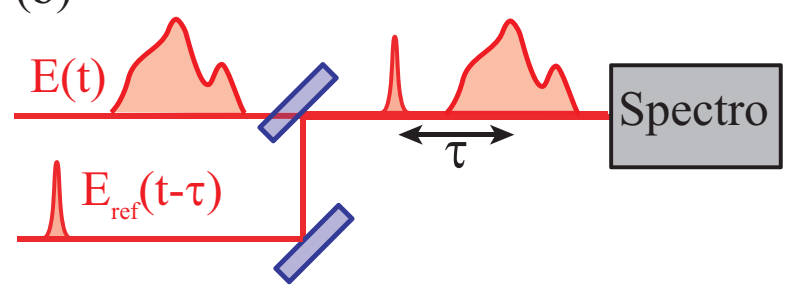

Figure 19. Spectral interferometry: The test pulse $E(t)$ is sent into a spectrometer either (a) alone to measure spectral amplitude or (b) together with a delayed reference pulse $E_{r e f}(t-\tau)$ to retrieve the spectral phase.

a)

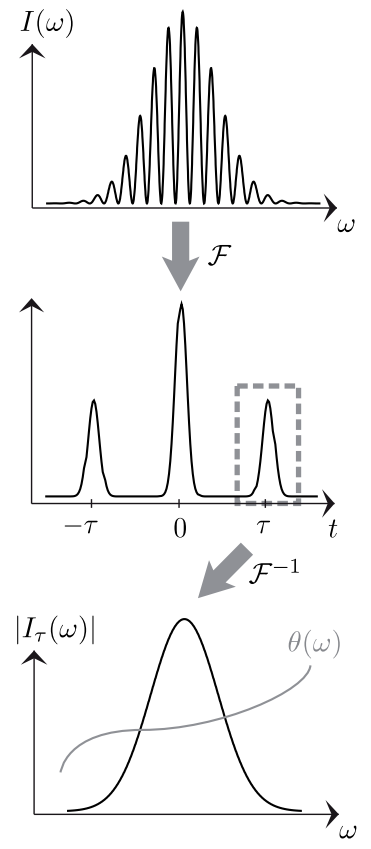

b)
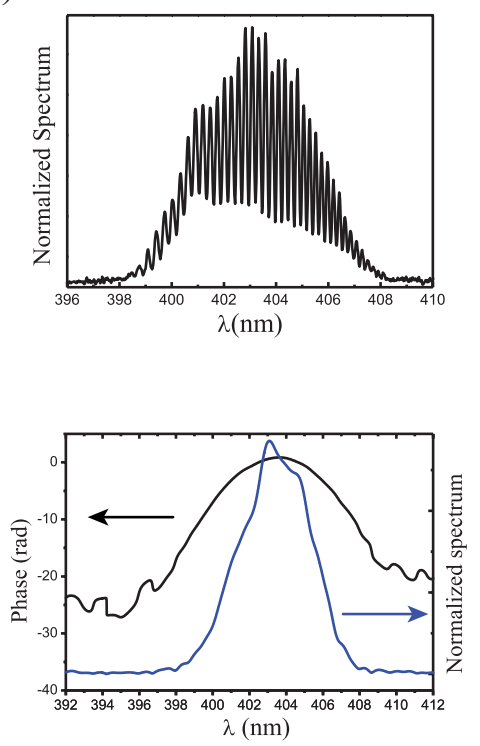

Figure 20. Principle of Fourier filtering for spectral interferometry. a) Theory. b) Experimental interferogram of a $10000 \mathrm{fs}^{2}$ quadratic phase shaped pulse and reconstructed amplitude and phase.

\subsubsection{Spectral interferometry}



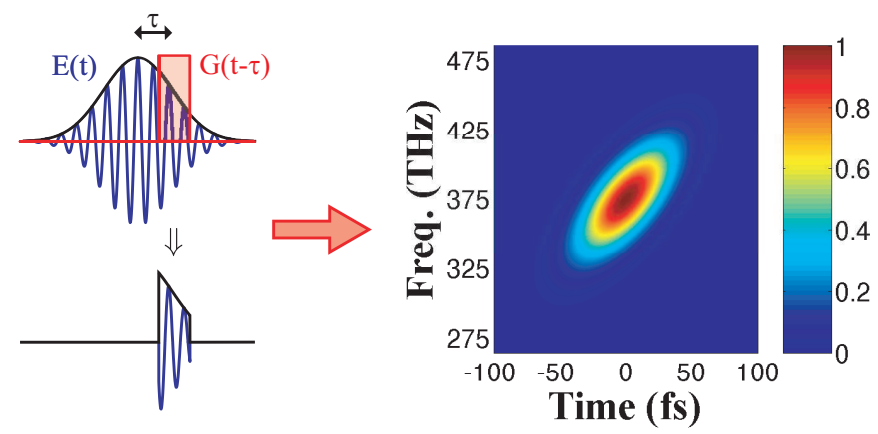

Figure 21. Principle of Frequency Optical Gating :The test pulse $E(t)$ is temporally sliced (or gated). The spectrum of this gated pulse is measured as a function of the delay $\tau$ between the gate $G(t)$ and the test pulse.

\subsubsection{Fourier transform spectroscopy}

\subsection{Self-referenced complete characterization methods}

4.3.1. Prerequisite for self-referenced measurement of $\varphi(\omega)$

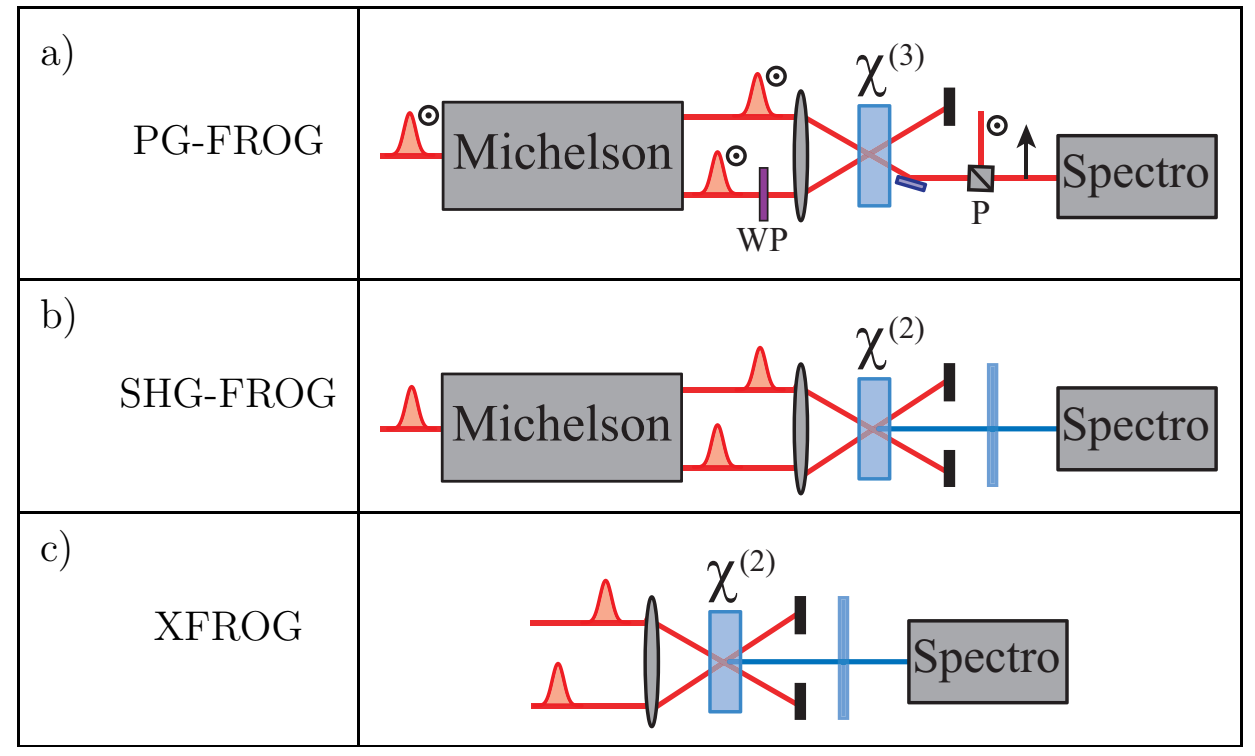

Table 4. Schematic setup of various versions of FROG techniques. a) Polarization gating FROG, b) Second harmonic generation FROG and c) Crosscorrelation FROG. WP is a wave plate to tune the polarization, $\mathrm{P}$ is a polarizer, $\chi^{(2)}$ and $\chi^{(3)}$, non-linear susceptibilities.

\subsubsection{FROG and other variants}




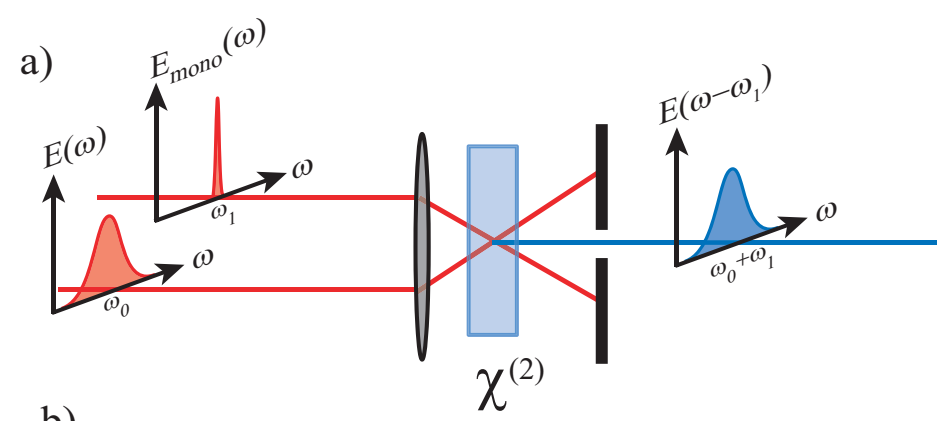

b)

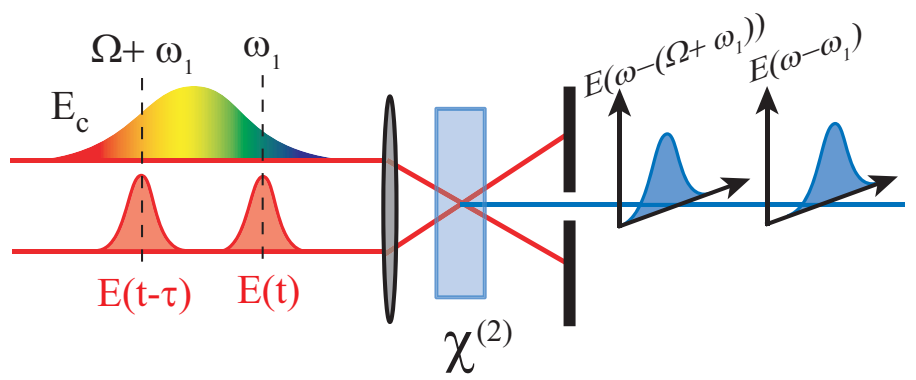

Figure 22. Spectral shearing by upconversion: (a) the upconversion between $E(\omega)$ a quasi-monochromatic pulse $E_{\text {mono }}(\omega)$ centered around $\omega_{1}$ generates a spectrally shifted replica $E\left(\omega-\omega_{1}\right)$; (b) the upconversion between two delayed replicas $E(t)$ and $E(t-\tau)$ and a chirped pulse $E_{c}(t)$ leads to two delayed and spectrally shifted replicas.

$P G F R O G$

SHG FROG

XFROG

Closing words on FROG

4.3.3. SPIDER and other variants

The original flavour of SPIDER

Zero Additional Phase SPIDER 


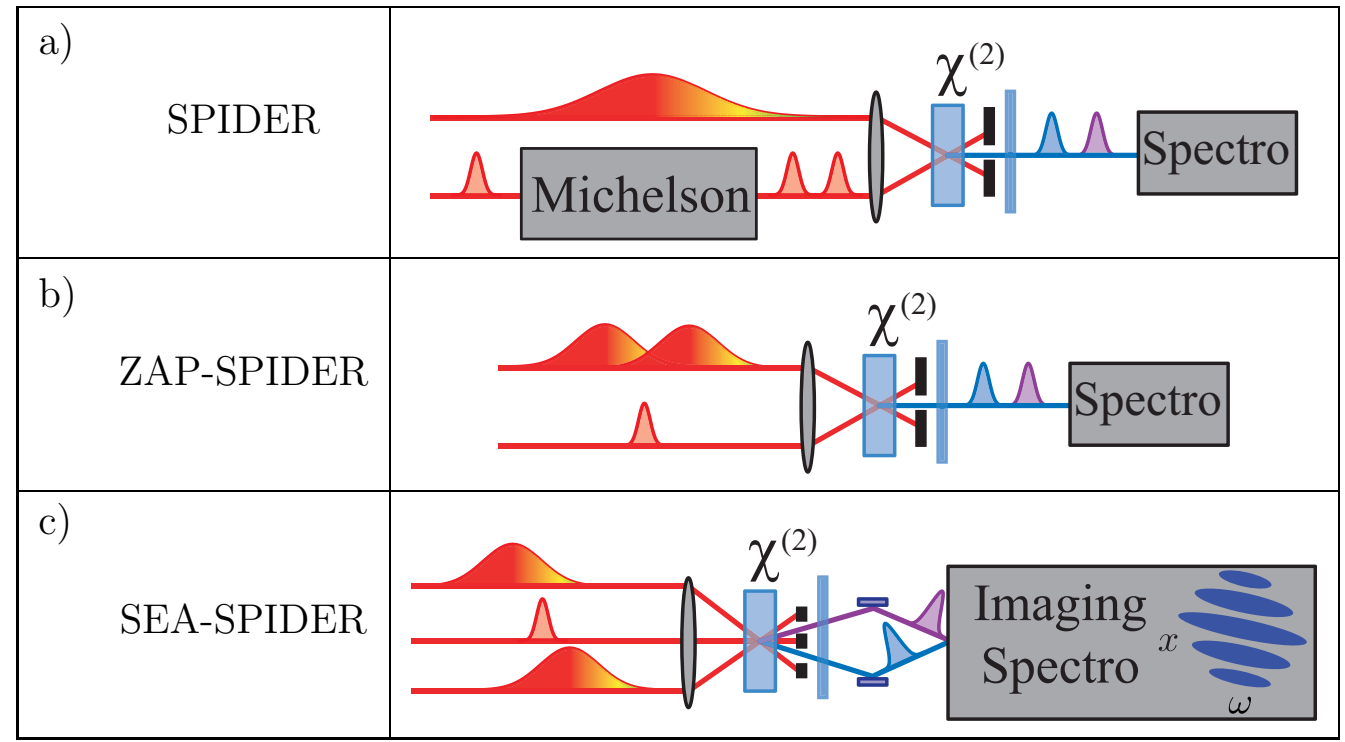

Table 5. Different SPIDER techniques and their schematic setups. a) Original SPIDER, b) Zero Additional Phase SPIDER and c) Spatially Encoded Arrangement SPIDER. $\chi^{(2)}$ denotes the non-linear susceptibility.

Spatial Encoded Arrangement for SPIDER

Closing word on SPIDER 


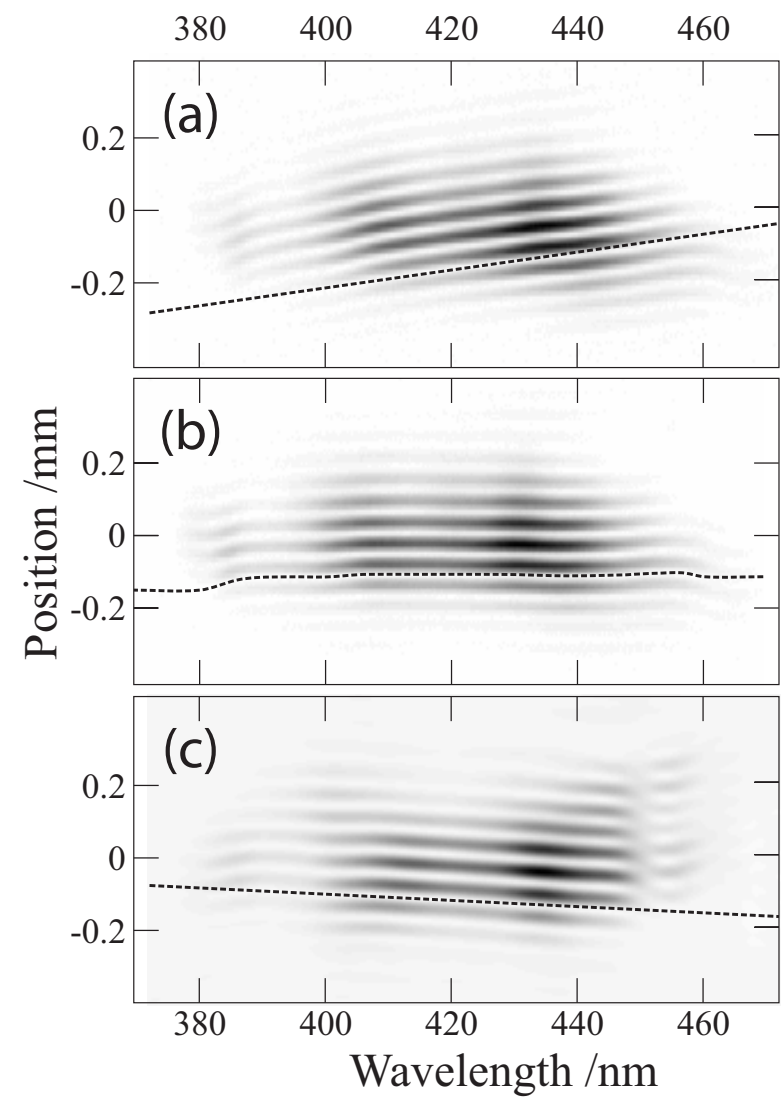

Figure 23. SEA SPIDER intuitive traces: SEA SPIDER traces for a Femtolaser Rainbow oscillator, (a) for a positive $\varphi_{0}^{(2)}$, (b) for a recompressed pulse and (d) for a negative chirp. The small wiggles in (b) are a signature of small remaining high order phase.

\section{The combination of pulse shaping and characterization techniques}

\section{General conclusion}

\section{Bibliography}

[1] Matthew Fetterman, Debabrata Goswami, Dorine Keusters, Weiguo Yang, June-Koo Rhee, and Warren Warren. Ultrafast pulse shaping: amplification and characterization. Opt. Express, 3(10):366-375, 1998.

[2] M. A. Dugan, J. X. Tull, and W. S. Warren. High-resolution acousto-optic shaping of unamplified and amplified femtosecond laser pulses. Journal of the Optical Society of America B (Optical Physics), 14(9):2348-58, 1997.

[3] S.H. Shim, D. B. Strasfeld, E.C. Fulmer, and M.T. Zanni. Femtosecond pulse shaping directly in the mid-ir using acousto-optic modulation. Opt. Lett., 31:838, 2006.

[4] W. S. Warren, H. Rabitz, and M. Dahleh. Coherent control of quantum dynamics: The dream is alive. Science, 259:1581, 1993.

[5] A. Monmayrant and B. Chatel. A new phase and amplitude high resolution pulse shaper. Rev. Sci. Instr., 75(8):2668-71, 2004.

[6] A. PrÃ̃d'kelt, M. Wollenhaupt, A. Assion, C. Horn, C. Sarpe-Tudoran, M. Winter, and T. Baumert. 
Compact, robust and flexible setup for femtosecond pulse shaping. Rev. Sci. Instr., 74(11):4950, 2003.

[7] G. Stobrawa, M. Hacker, T. Feurer, D. Zeidler, M. Motzkus, and F. Reichel. A new high-resolution femtosecond pulse shaper. Applied Physics B, 72(5):627-30, 2001.

[8] M. M. Wefers and K. A. Nelson. Programmable phase and amplitude femtosecond pulse shaping. Opt. Lett., 18(23):2032, 1993.

[9] T. Brixner and G. Gerber. Femtosecond polarization pulse shaping. Optics Letters, 26(8):557-9, 2001.

[10] E. Frumker, D. Oron, D. Mandelik, and Y. Silberberg. Femtosecond pulse-shape modulation at kilohertz rates. Optics Letters, 29(8):890-892, 2004.

[11] D. Zeidler, T. Witte, D. Proch, and M. Motzkus. Optical parametric amplification of a shaped white-light continuum. Optics Letters, 26(23):1921-3, 2001.

[12] C. W. Hillegas, J. X. Tull, D. Goswami, D. Strickland, and W. S. Warren. Femtosecond laser pulse shaping by use of microsecond radio-frequency pulses. Opt. Lett., 19(10):737, 1994 .

[13] Howe Siang Tan, W. S. Warren, and E. Schreiber. Generation and amplification of ultrashort shaped pulses in the visible by a two-stage noncollinear optical parametric process. Opt. Lett., 26(22):1812, 2001.

[14] B. Pearson and T.C. Weinacht. Shaped ultrafast laser pulses in the deep ultraviolet. optics express, 17(7):4385, 2007.

[15] M. Roth, M. Mehendale, A. Bartelt, and H. Rabitz. Acousto-optical shaping of ultraviolet femtosecond pulses. Applied Physics B: Lasers and Optics, 80(4-5):441, 2005.

[16] E. Zeek, K. Maginnis, S. Backus, U. Russek, M. Murnane, G. Mourou, H. Kapteyn, and G. Vdovin. Pulse compression by use of deformable mirrors. Opt. Lett., 24(7):493, 1999.

[17] M. Hacker, G. Stobrawa, R. Sauerbrey, T. Buckup, M. Motzkus, M. Wildenhain, and A. Gehner. Micromirror slm for femtosecond pulse shaping in the ultraviolet. Applied Physics B-Lasers And Optics, 76(6):711-714, 2003.

[18] N. Belabas, J.P. Likforman, L. Canioni, B. Bousquet, and M. Joffre. Coherent broadband pulse shaping in the mid infrared. Opt. Lett., 26:743, 2001.

[19] T. Witte, K.L. Kompa, and M. Motzkus. Femtosecond pulse shaping in the mid infrared by difference-frequency mixing. Applied Physics B, 76(4):467-471, 2003.

[20] H. S. Tan, E. Schreiber, and W. S. Warren. High-resolution indirect pulse shaping by parametric transfer. Opt. Lett., 27(6):439, 2002.

[21] **C. Schriever, S. Lochbrunner, M. Optiz, and E. Riedle. 19 fs shaped ultraviolet pulses. Opt. Lett., 31(4):543, 2006.

[22] P. Tournois. Design of acousto-optic programmable filters in mercury halides for mid-infrared laser pulse shaping. Optics Communication, 281:4054, 2008.

[23] ${ }^{* *}$ F. Verluise, V. Laude, Z. Cheng, C. Spielmann, and P. Tournois. Amplitude and phase control of ultrashort pulses by use of an acousto-optic programmable dispersive filter: pulse compression and shaping. Opt. Lett., 25(8):575, 2000.

[24] Antoine Monmayrant, Arnaud Arbouet, Bertrand Girard, BÃl'atrice Chatel, Benjamin J. Whitaker, A. Barman, and Daniel Kaplan. Optimisation of nopa output pulse shaping using an aopdf with dispersion self-correction. Appl. Phys. B, 81(2-3):177, 2005. http://fr.arxiv.org/pdf/physics/0505152.

[25] Sebastien Coudreau, Daniel Kaplan, and Pierre Tournois. Ultraviolet acousto-optic programmable dispersive filter laser pulse shaping in kdp. Opt. Lett., 31(12):1899, 2006.

[26] S. Weber, M. Barthelemy, and B. Chatel. Direct shaping of tunable uv ultrashort pulses. optics express submitted, 2009. 